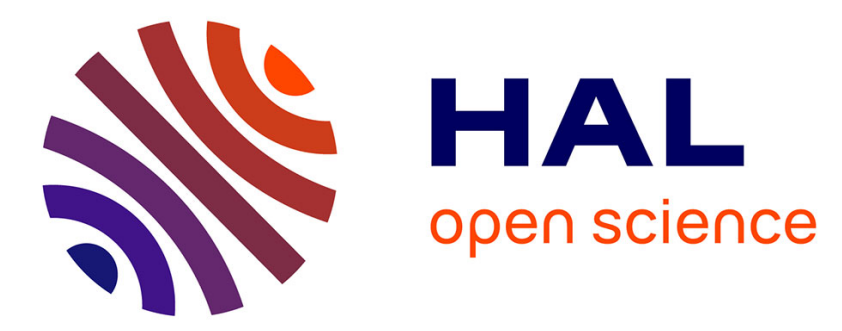

\title{
Catalytic Synthesis of Trifluoromethylated Allenes, Indenes, Chromenes, and Olefins from Propargylic Alcohols in HFIP
}

Florent Noël, Vuk D. Vuković, Jing Yi, Edward Richmond, Pavle Kravljanac, Joseph Moran

\section{To cite this version:}

Florent Noël, Vuk D. Vuković, Jing Yi, Edward Richmond, Pavle Kravljanac, et al.. Catalytic Synthesis of Trifluoromethylated Allenes, Indenes, Chromenes, and Olefins from Propargylic Alcohols in HFIP. Journal of Organic Chemistry, 2019, 84 (24), pp.15926-15947. 10.1021/acs.joc.9b02398 . hal-02516523

\section{HAL Id: hal-02516523 \\ https://hal.science/hal-02516523}

Submitted on 23 Mar 2020

HAL is a multi-disciplinary open access archive for the deposit and dissemination of scientific research documents, whether they are published or not. The documents may come from teaching and research institutions in France or abroad, or from public or private research centers.
L'archive ouverte pluridisciplinaire HAL, est destinée au dépôt et à la diffusion de documents scientifiques de niveau recherche, publiés ou non, émanant des établissements d'enseignement et de recherche français ou étrangers, des laboratoires publics ou privés. 


\title{
Catalytic Synthesis of Trifluoromethylated Allenes, Indenes, Chromenes and Olefins from Propargylic Alcohols in HFIP
}

Florent Noël, Vuk D. Vuković, Jing Yi, Edward Richmond, Pavle Kravljanac and Joseph Moran*

Université de Strasbourg, CNRS, ISIS, 8 allée Gaspard Monge, 67000 Strasbourg, France moran@unistra.fr
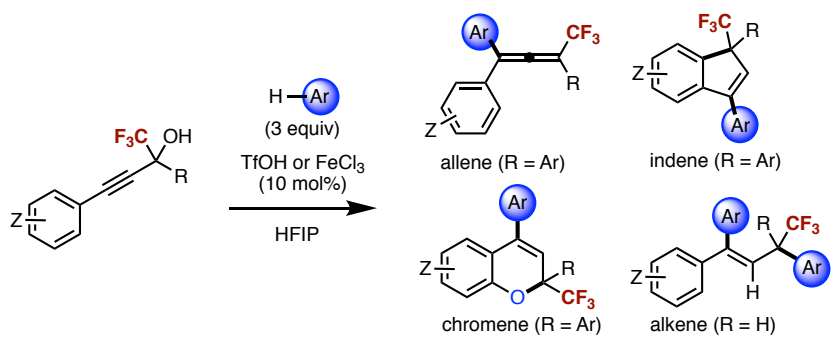

\begin{abstract}
A general method to access $\mathrm{CF}_{3}$-substituted allenes from propargylic alcohols under Lewis acid catalysis in 1,1,1,3,3,3-hexafluoro-2-propanol (HFIP) as solvent is described. By tuning the reaction time and temperature, the obtained allenes rearrange to 1,3-biaryl-1-trifluoromethyl-1 $H$ indenes. By tuning the structure of the propargylic alcohol substrates, a range of trifluoromethylated $2 \mathrm{H}$-chromenes were successfully synthesized, with use of catalytic quantities of strong Brønsted acid in HFIP. The present method is therefore highly potent for synthesis of a number of potentially pharmaceutically interesting new trifluoromethylated compounds and produces water as the only stoichiometric byproduct.
\end{abstract}

\section{INTRODUCTION}

Propargylic alcohols represent an attractive class of starting compounds for a number of chemical transformations. ${ }^{1}$ The direct nucleophilic substitution of propargylic alcohols can result in two types of products: an $\alpha$-substituted alkyne or an allene (Scheme 1a). ${ }^{2}$ The transformations leading to alkynes are well studied. ${ }^{3}$ On the other hand, there are few examples of the formation of allenes from propargyl alcohols through carbocationic intermediates. ${ }^{4}$ Generally, carbocationic 
intermediates bearing an adjacent electron-withdrawing group, such as a $\mathrm{CF}_{3}$ group, are difficult to form. They are typically generated from halides or pseudo-halides. ${ }^{5}$ In some cases, they have been generated from alcohols in the presence of superstoichiometric quantities of Brønsted or Lewis acids. ${ }^{6,7}$ Catalytic methods for their generation are desirable because substituting hydrogen atoms for fluorine atoms is well known to modulate a molecule's physico-chemical and pharmaceutically relevant properties, ${ }^{8}$ such as its pharmacokinetics and binding affinity. ${ }^{9}$ One of the most suitable organic solvents used to carry out reactions involving short-lived carbocations is 1,1,1,3,3,3-hexafluoroisopropanol (HFIP). ${ }^{10}$ We recently reported that Brønsted acid catalyzed Friedel-Crafts reactions of $\alpha$-trifluoromethylated benzylic alcohols proceed with ease via $\alpha$ trifluoromethyl carbocationic species in HFIP. ${ }^{11}$ As a continuation of this research program, we explored the reactivity of $\alpha$-trifluoromethylated propargylic alcohols in HFIP under Brønsted and Lewis acid catalysis. Here we show that $\mathrm{CF}_{3}$-bearing propargyl alcohols are versatile substrates in dehydroarylative Friedel-Crafts reactions (Scheme 1b), giving access to a number of trifluoromethylated allenes, indenes, chromenes and alkenes. The method is of use for the diversity-oriented synthesis of trifluoromethylated compounds, which are of considerable interest to pharmaceutical research, ${ }^{12}$ and produces water as the only stoichiometric byproduct.

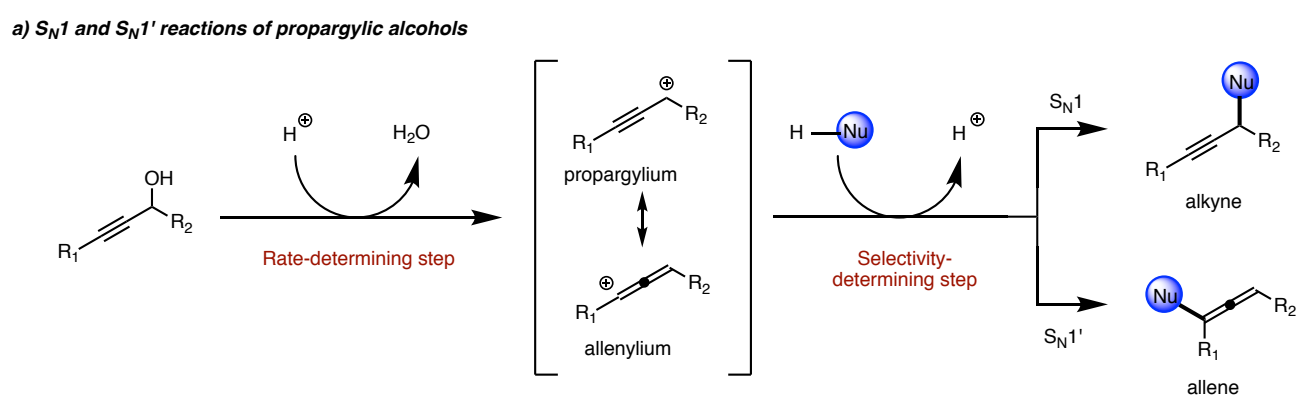

b) This work: HFIP-assisted transformations of a-CF 3 propargyl alcohols

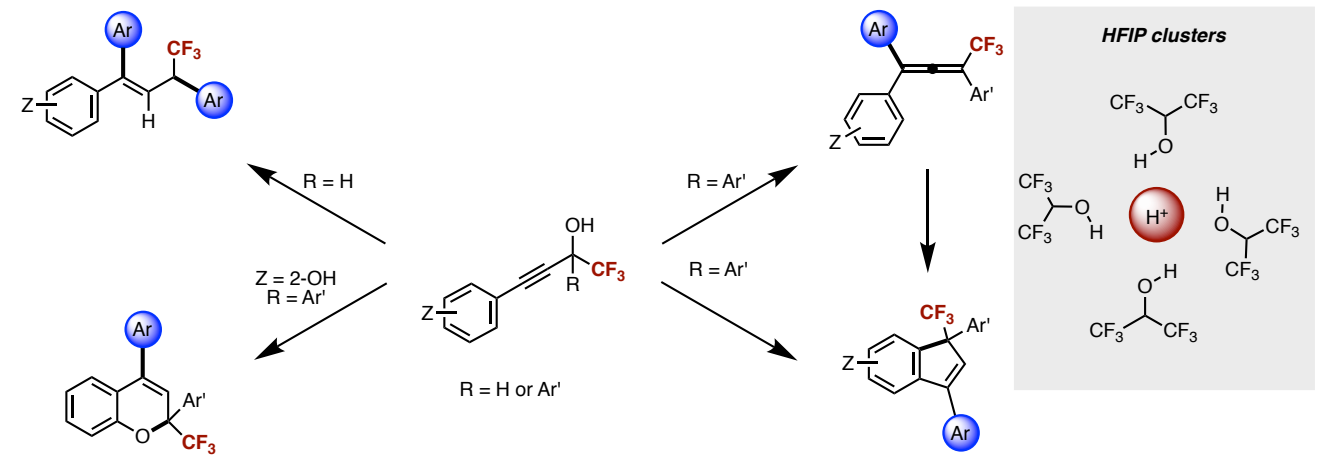

Scheme 1. HFIP-assisted transformation of $\alpha-\mathrm{CF}_{3}$ propargyl alcohols. 
Methods for the synthesis of allenes bearing electron-withdrawing groups, such as keto, carboxy, ester, amide, cyano, sulfone groups have been described. ${ }^{13}$ Among them, only a limited number allow access to monoary ${ }^{14}$ and biary ${ }^{15}$ mono $\left(\mathrm{CF}_{3}\right)$ substituted allenes (Scheme $2 \mathrm{a}$ ). A single example of the Pd-catalyzed synthesis of triaryl mono $\left(\mathrm{CF}_{3}\right)$ substituted allenes in known. ${ }^{16}$ To our knowledge, no general catalytic methods allowing access to triaryl mono $\left(\mathrm{CF}_{3}\right)$ substituted allenes have been reported so far. Furthermore, methods for $\mathrm{CF}_{3}$-allene synthesis proceeding via carbocationic intermediates have not been described.

Methods for the synthesis of indenes bearing $\mathrm{CF}_{3}$-groups at the $\mathrm{C} 1-\mathrm{C} 3$ positions are known but are limited in terms of the substitution patterns that can be achieved. Reported strategies include the treatment of indanones with perfluoroalkyl lithium reagents ${ }^{17}$ and the use of $\alpha$ (trifluoromethyl)allyl alcohols. ${ }^{18,19}$ In general, indenes can be prepared by the isomerization of allenes, ${ }^{20}$ but the preparation of $\mathrm{CF}_{3}$-indenes through the intermediacy of $\mathrm{CF}_{3}$-allenes, reported herein, had not been described. However, during the preparation of this manuscript, Vasilyev, Nenajdenko, Krasavin and collaborators reported a single example of the conversion of a propargylic alcohol to a $\mathrm{CF}_{3}$-indene using neat sulfuric or triflic acid as solvent, ${ }^{21}$ or zeolites ${ }^{22}$ under high pressure and temperature conditions (Scheme 2b). Herein we report the first general method for the synthesis of 1,3-biaryl-1-trifluoromethyl-1H-indenes, which employs a Brønsted acid catalyzed approach under significantly milder conditions. Moreover, by tuning of the reaction time and temperature, the corresponding intermediate triaryl $\mathrm{CF}_{3}$ allenes can be accessed. 


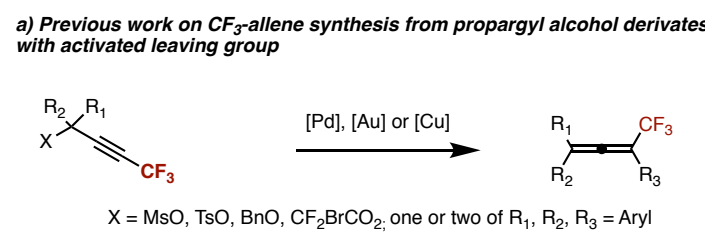

b) Recent work on $\mathrm{CF}_{3}$-indene synthesis from propargylic alcohols

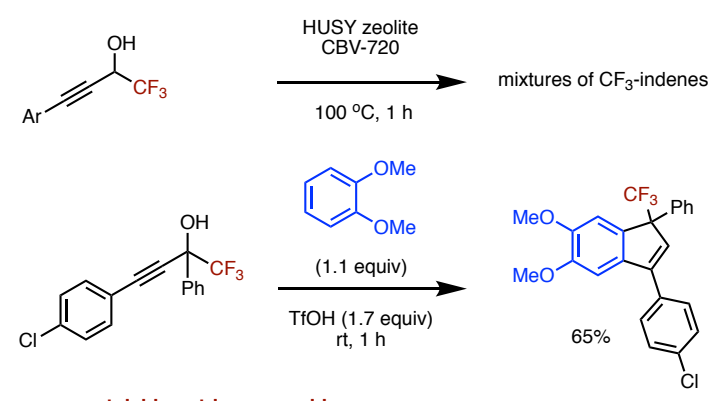

- superstoichiometric superacid

- only example of an 1,3-diaryl-1- $\mathrm{CF}_{3}$-1H-indene

obtained from a propargylic alcohol

Scheme 2. Previous reports on $\mathrm{CF}_{3}$-allene and -indene synthesis

\section{RESULTS AND DISCUSSION}

Reaction discovery and optimization. We began our investigations with standard conditions for dehydroarylative reactions of benzylic alcohols in HFIP that were previously established. ${ }^{11}$ Instead of direct nucleophilic substitution on the $\alpha-\mathrm{CF}_{3}$ carbon of alcohol $\mathbf{1}$, with TfOH as catalyst in HFIP at room temperature we observed the nucleophile attack on $\gamma$-carbon and formation of allene 1a (Table 1, entry 1). When the same reaction is conducted at higher temperature $\left(50{ }^{\circ} \mathrm{C}\right)$, the formation of an additional product ( $\mathrm{CF}_{3}$-substituted indene, 2a) was observed (entry 2). Weaker Brønsted acids did not lead to the formation of allene or indene (entries 3-5). The stronger fluoroantimonic acid gave a mixture of allene and indene in an approximate ratio of 11:1 (entry 6). Some Lewis acid catalysts provided the allene, but not the corresponding indene in significant yield (entries 7-11). However, $\mathrm{FeCl}_{3}$ led to the formation of the allene in $93 \%$ isolated yield in 10 min (entry 12). Heating at $80{ }^{\circ} \mathrm{C}$ after longer reaction times led to complete transformation to the corresponding indene (entry 13). By testing the same reaction in other solvents (entries 14-16), we confirmed that this reactivity is optimal in HFIP. Iron(II) chloride was not as efficient as iron(III) chloride (entry 17). 
Table 1. Reaction optimization and discovery

\begin{tabular}{|c|c|c|c|c|c|}
\hline entry & catalyst & solvent & time & $\begin{array}{l}\text { yield 1a } \\
(\%)\end{array}$ & $\begin{array}{l}\text { yield 2a } \\
(\%)\end{array}$ \\
\hline 1 & TfOH & HFIP & $1 \mathrm{~h}$ & 85 & - \\
\hline $2^{\mathrm{a}}$ & TfOH & HFIP & $45 \mathrm{~min}$ & 50 & 43 \\
\hline $3^{a}$ & TFA & HFIP & $24 \mathrm{~h}$ & 60 & - \\
\hline 4 & $\mathrm{H}_{3} \mathrm{PO}_{4}$ & HFIP & $24 \mathrm{~h}$ & - & - \\
\hline 5 & $\mathrm{HCl}^{\mathrm{b}}$ & HFIP & $10 \mathrm{~min}$ & traces & - \\
\hline 6 & $\mathrm{HSbF}_{6} \cdot 6 \mathrm{H}_{2} \mathrm{O}$ & HFIP & $10 \mathrm{~min}$ & 87 & 8 \\
\hline 7 & $\mathrm{Sc}(\mathrm{OTf})_{3}$ & HFIP & $24 \mathrm{~h}$ & 9 & - \\
\hline 8 & $\mathrm{SbF}_{5}$ & HFIP & $10 \mathrm{~min}$ & 66 & - \\
\hline $9^{\mathrm{a}}$ & $\mathrm{AlCl}_{3}$ & HFIP & $45 \mathrm{~min}$ & 24 & 10 \\
\hline 10 & $\mathrm{ZnCl}_{2}$ & HFIP & $24 \mathrm{~h}$ & 7 & - \\
\hline 11 & $\mathrm{AuCl}_{3}$ & HFIP & $10 \mathrm{~min}$ & 69 & - \\
\hline 12 & $\mathrm{FeCl}_{3}$ & HFIP & $10 \mathrm{~min}$ & 93 & - \\
\hline $13^{\mathrm{c}}$ & $\mathrm{FeCl}_{3}$ & HFIP & $24 \mathrm{~h}$ & - & 94 \\
\hline 14 & $\mathrm{FeCl}_{3}$ & $i$-PrOH & $10 \mathrm{~min}$ & - & - \\
\hline 15 & $\mathrm{FeCl}_{3}$ & $\mathrm{CF}_{3} \mathrm{CH}_{2} \mathrm{OH}$ & $10 \mathrm{~min}$ & 39 & - \\
\hline 16 & $\mathrm{FeCl}_{3}$ & $\mathrm{CH}_{2} \mathrm{Cl}_{2}$ & $24 \mathrm{~h}$ & 9 & - \\
\hline 17 & $\mathrm{FeCl}_{2}$ & HFIP & $24 \mathrm{~h}$ & 41 & - \\
\hline
\end{tabular}

Scope of trifluoromethylated allenes. Encouraged by the initial results, the scope of triaryl monotrifluoromethyl allenes was explored (Table 2). The parent 1,1,1-trifluoro-2,4-diphenyl-but3-yn-1-ol (1) furnished the corresponding allene 1a with mesitylene as nucleophile in $93 \%$ yield. Replacement of the phenyl ring A with cyclohexyl (1b) or biphenyl (1c) groups lead to a significant decrease in yield, but with $p$-tolyl substitution this decrease was less pronounced (1d). However, $o$-methyl substitution of ring A (1e) or $p$-methyl substition of ring B (1f) did not influence the yield significantly. $p$-Bromo substitution of phenyl rings A or B slowed down the reaction (1g and $\mathbf{1 h}$ ), which could be overcome in most cases by increasing the reaction time (1h). As expected, an electron-withdrawing ( $p$-fluoro) substituent on phenyl ring B had a deactivating influence (1i). However, electron-donating ( $p$-methoxy) groups also led to lower yields, due to rapid subsequent 


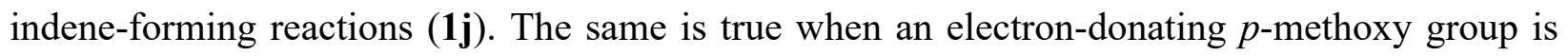
present on ring A. For example, attempts to isolate allene $1 \mathbf{k}$ could only be accomplished in $27 \%$ yield by interrupting the reaction after 5 minutes. In these cases, attempts to isolate the allene by running at the reaction at lower temperatures or under different concentrations were unsuccessful since the subsequent cyclization is more facile than the initial allene formation. Finally, the use of bulkier nucleophiles such as 1,4-diisopropylbenzene also lead to decreased yields (11-10) as did less activated (Cl- and F- substituted) nucleophiles (1p-1r). To confirm the structure of the allenes, we obtained an X-ray crystal structure of allene $\mathbf{1 s .}$

Table 2. Scope of tetrasubstituted allenes bearing a $\mathrm{CF}_{3}$ group
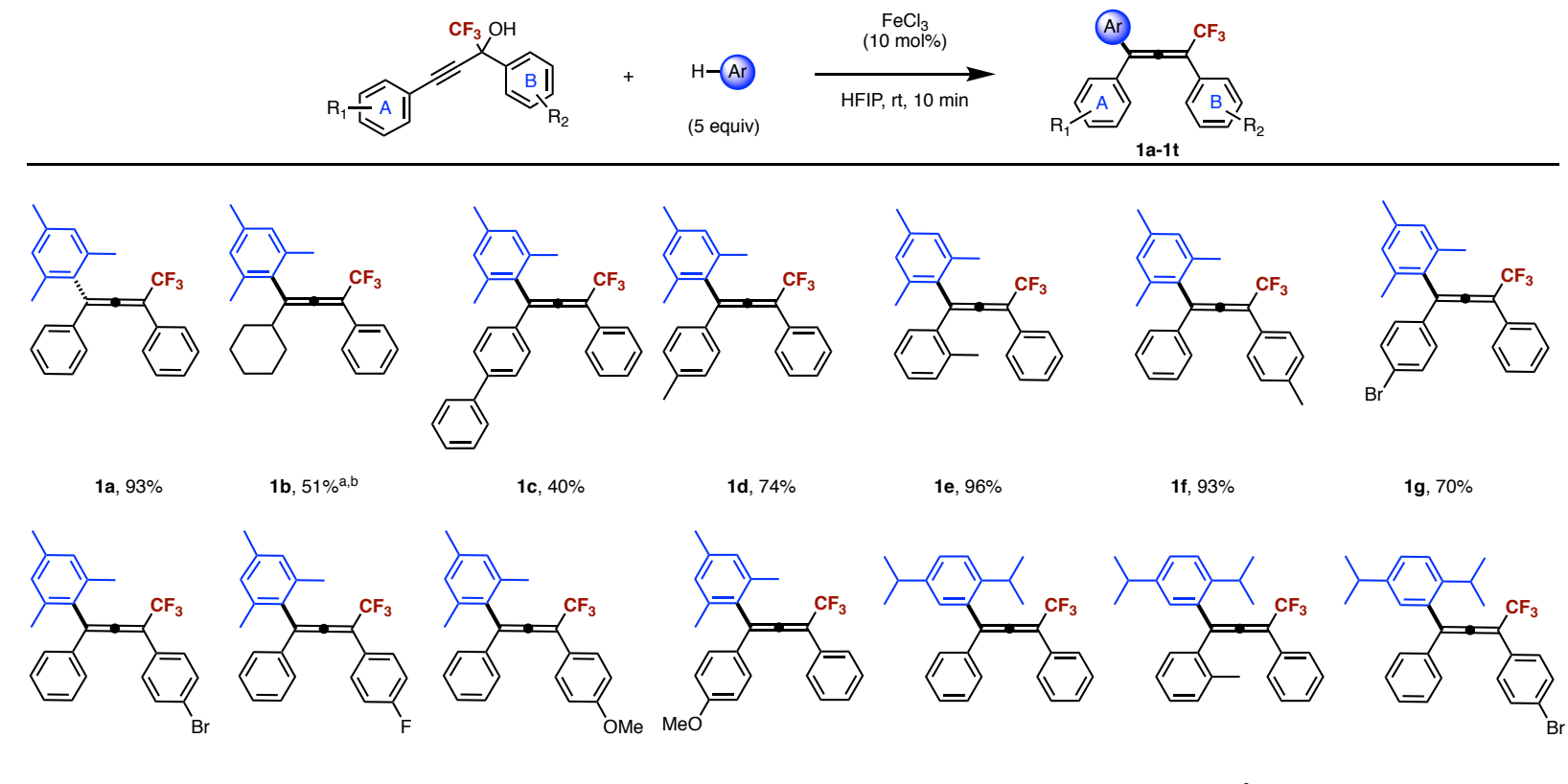

$1 \mathrm{~h}, 90 \%^{\mathrm{c}}$

$1 \mathrm{j}, 82 \%$

1 k, $27 \%{ }^{\mathrm{e}}$

1I, $65 \%$

$1 \mathrm{~m}, 56 \%^{\mathrm{c}}$

1n, $40 \%$

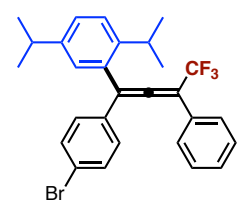

1o, $70 \%^{\mathrm{c}}$

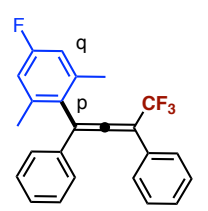

1p, $25 \%$ and 1 q, $28 \%$ b,f

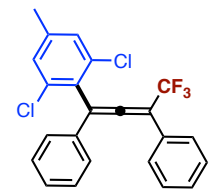

1 r, $25 \%$ b,f

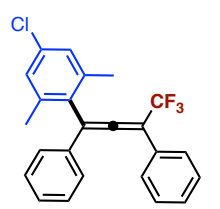

1s, $25 \%$ b,f

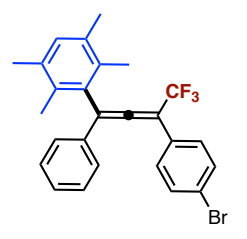

1t, $93 \%{ }^{\mathrm{c}, \mathrm{f}}$

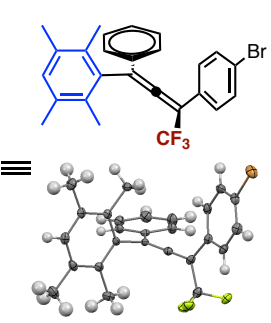

${ }^{a}$ Heated at $50{ }^{\circ} \mathrm{C} .{ }^{b}$ Reaction time was $24 \mathrm{~h} .{ }^{c}$ Reaction time was $3 \mathrm{~h} .{ }^{d}$ Reaction time was $1 \mathrm{~h}$. ${ }^{e}$ Reaction time was $5 \mathrm{~min} .{ }^{f}$ Heated at $80{ }^{\circ} \mathrm{C}$. 
Scope of trifluoromethylated indenes. In the next step of the study, the scope of indenes was explored (Table 3). The indenes $\mathbf{2 a}$ and $\mathbf{2 l}$ were obtained via allenes $\mathbf{1 a}$ and $\mathbf{1 1}$ in excellent yields. The indenes $\mathbf{2 c}, \mathbf{2 d}$ and $\mathbf{2 f}$ substituted with phenyl or methyl groups were all obtained in excellent yields. When a methoxy group is attached in the para position on the phenyl ring $\mathrm{B}$, the yield of the indene $\mathbf{2} \mathbf{j}$ remained high, unlike the case of the para substituted phenyl ring A (2k). The reaction was slown down when a bromo-substituent was introduced in the phenyl ring B (2n). 1,3,5-Triethylbenzene proved to be a good nucleophile for formation of indenes (entry $\mathbf{2} \mathbf{x}$ ). By increasing the temperature to $120{ }^{\circ} \mathrm{C}$, it was possible to use 1,3,5-trimethoxybenzene as a nucleophile (entry $\mathbf{2 y}$ ). However, when a chloro-bearing arene was used, the yield dropped significantly (2s). In the case of indene $\mathbf{2 x}$ and $\mathbf{2 y}$, we were unable to isolate the intermediate allene. Generally, attempts to form indenes from propargylic alcohols using weaker arene nucleophiles via allenes were unsuccessful (for example, via allenes 1p-1q), due both to the reduced ability of the nucleophile to capture the propargylic carbocation, as well as the subsequent deactivating effect of the electron-poor arene on the cyclization of the allene intermediate. Even under more forcing conditions, propargylic alcohols bearing a bromide or fluoride on ring A or ring $\mathrm{B}$ did not lead to indenes, but instead stopped at the allenes $(\mathbf{1 g}, \mathbf{1 h}, \mathbf{1 i}, \mathbf{1 0}, \mathbf{1 s})$ previously described in Table 2. 


\section{Table 3. Scope of indenes bearing a $\mathrm{CF}_{3}$ group}
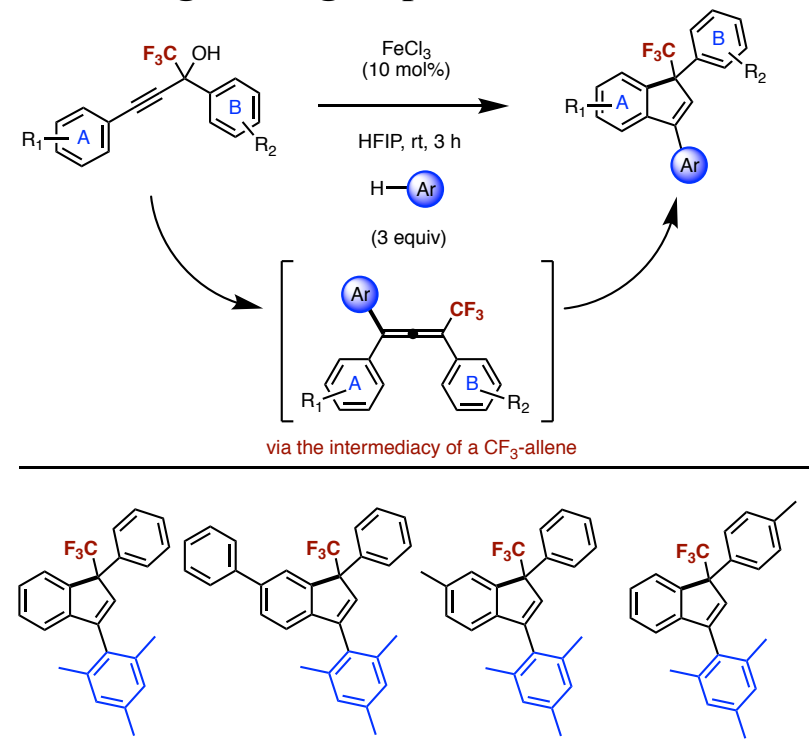

$\begin{array}{llll}\mathbf{2 a}, 94 \%^{\mathrm{a}, \mathrm{b}} & \mathbf{2 c}, 91 \%^{\mathrm{c}} & \mathbf{2 d}, 91 \%^{\mathrm{c}} & \mathbf{2 f}, 96 \%\end{array}$

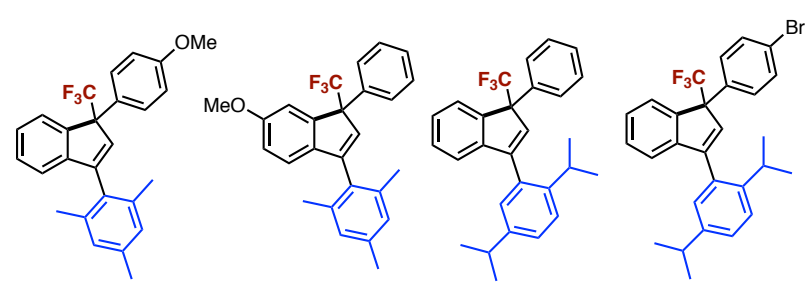

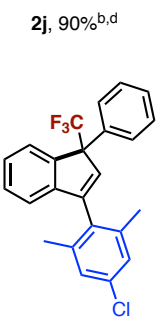

2s, $19 \%$ a,b,e

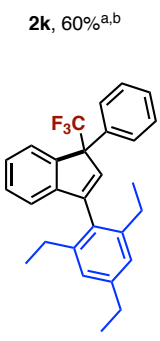

$2 \mathbf{x}, 93 \%$ a,b

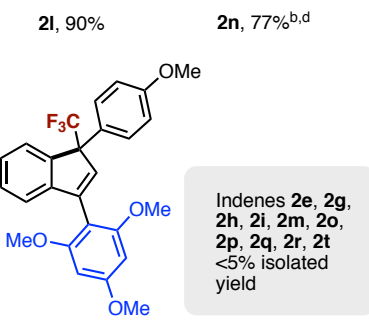

$2 y, 95 \%$ a,

${ }^{a}$ Reaction time was 24 h. ${ }^{b}$ Heated at $80{ }^{\circ} \mathrm{C}$. ${ }^{c}$ Carried out at $50{ }^{\circ} \mathrm{C}$ for $6 \mathrm{~h} .{ }^{d}$ Reaction time was $1 \mathrm{~h}$. ${ }^{e}$ Isolated as mixture of regioisomers. ${ }^{f}$ Heated at $120{ }^{\circ} \mathrm{C}$.

Access to $2 \mathrm{H}$-2-trifluoromethyl chromenes. We hypothesized that propargylic alcohols with an $o$-hydroxyphenyl moiety should give access to corresponding $2 \mathrm{H}$-2-trifluoromethyl chromenes. Chromenes (benzopyrans) are a class of organic compounds that exhibit various biological activities, ${ }^{23}$ especially their trifluoromethylated derivates. ${ }^{24}$ Here, we found that the catalytic use of $\mathrm{TfOH}$ gave higher yields than $\mathrm{FeCl}_{3}$. Indeed, when 1,1,1-trifluoro-4-(2-hydroxophenyl)-2phenylbut-3-yn-1-ol (3a) was subjected to $10 \mathrm{~mol} \% \mathrm{TfOH} / \mathrm{in} \mathrm{HFIP},{ }^{25}$ we were pleased to observe formation of chromene $\mathbf{4 a}$ in quantitative yield (Table 4). Other nucleophiles such as durene, 
pentamethylbenzene and 1,3,5-trimethoxybenzene proved to be compatible with the reaction conditions (4b-4d). Slight modifications to the electronic properties of the 2-phenyl ring (4e and 4f) or replacement of the 2-aryl moiety with a 2-alkyl group (4g) were tolerated. Furthermore, the tert-butyl-dimethylsilyl ether derivatives $\mathbf{3} \mathbf{e}^{\prime}$ and $\mathbf{3 f}$ yielded the same product as their phenolic precursors (3e), due to an in situ deprotection/cyclization on $1 \mathrm{mmol}$ scale.

Table 4. Scope of chromenes bearing trifluoromethyl group

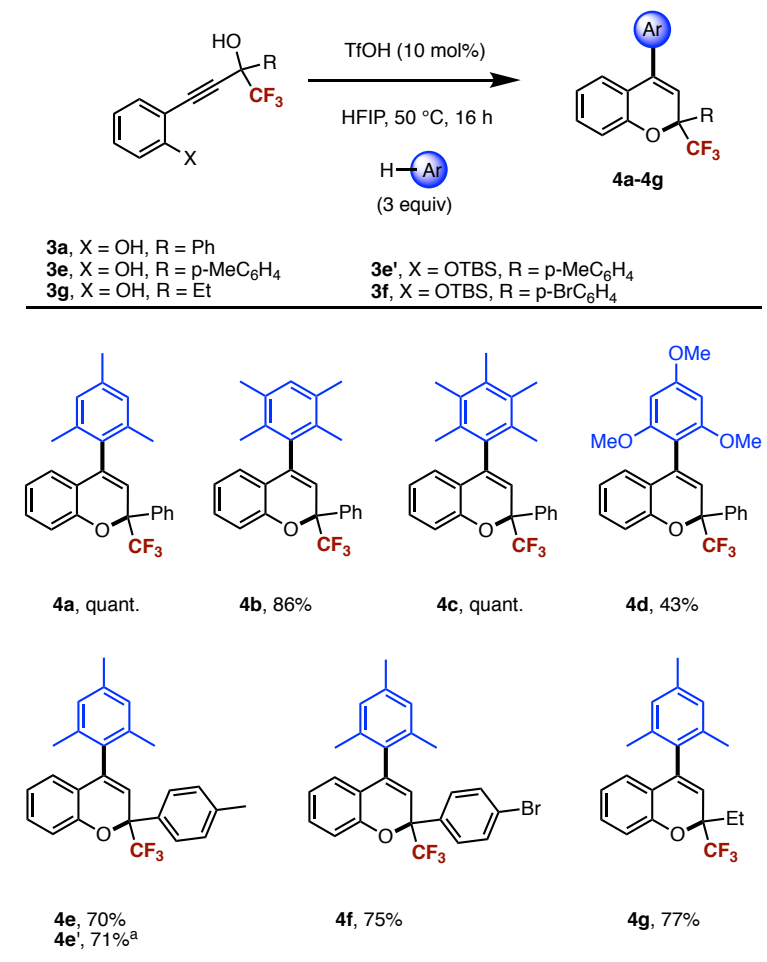

${ }^{a}$ Performed on $1 \mathrm{mmol}$ scale.

Reactivity of secondary $\alpha$-trifluoromethyl propargylic alcohols in HFIP. In light of the successful reactions of tertiary $\mathrm{CF}_{3}$-propargylic alcohols, we next tested the reactivity of secondary $\mathrm{CF}_{3}$-propargylic alcohols in the presence of catalytic $\mathrm{TfOH}$. Unexpectedly, by applying the same reaction conditions, a bis-arylated alkene 6a was observed (Table 5). An X-ray crystal structure of the product $\mathbf{6 a}$ revealed the $Z$-geometry of the double bond, as well as a preferred conformation in which the two mesityl units are aligned. A series of para-substituted secondary $\alpha$-trifluoromethyl propargylic alcohols was tested for Friedel-Crafts reactions with methyl- and methoxy-substituted benzenes as nucleophiles (Table 5). Methyl- (5b) and methoxy- 
(5c) substituted propargyl alcohols furnished products $\mathbf{6 b}$ and $\mathbf{6 c}$ in slightly higher yields than the parent alcohol 6a. Substitution with a cyano-group led to significant loss in reactivity (6d). In addition to the electronic deactivating effect of the $\mathrm{CN}$ group, the lowered yield may also potentially be due to the ability of this mildly basic group to buffer the Brønsted acid catalyst. The less electron-withdrawing bromine substituent led only to a minor drop in yield (6e). When 1,3,5trimethoxybenzene was used as nucleophile, substantially lower yield of $\mathbf{6} \mathbf{f}$ was obtained with the parent alcohol 5a. However, methoxy-substituted alcohol $\mathbf{5 c}$ furnished the corresponding product $\mathbf{6 g}$ in good yield. With other methyl-substituted benzenes, such as $p$-xylene (6h), pentamethylbenzene $(\mathbf{6 i - j})$ and durene $(\mathbf{6 k})$, as nucleophiles, reaction products were also obtained in good to excellent yields.

Table 5. Bis-addition to secondary $\alpha$-trifluoromethyl propargylic alcohols ${ }^{a}$
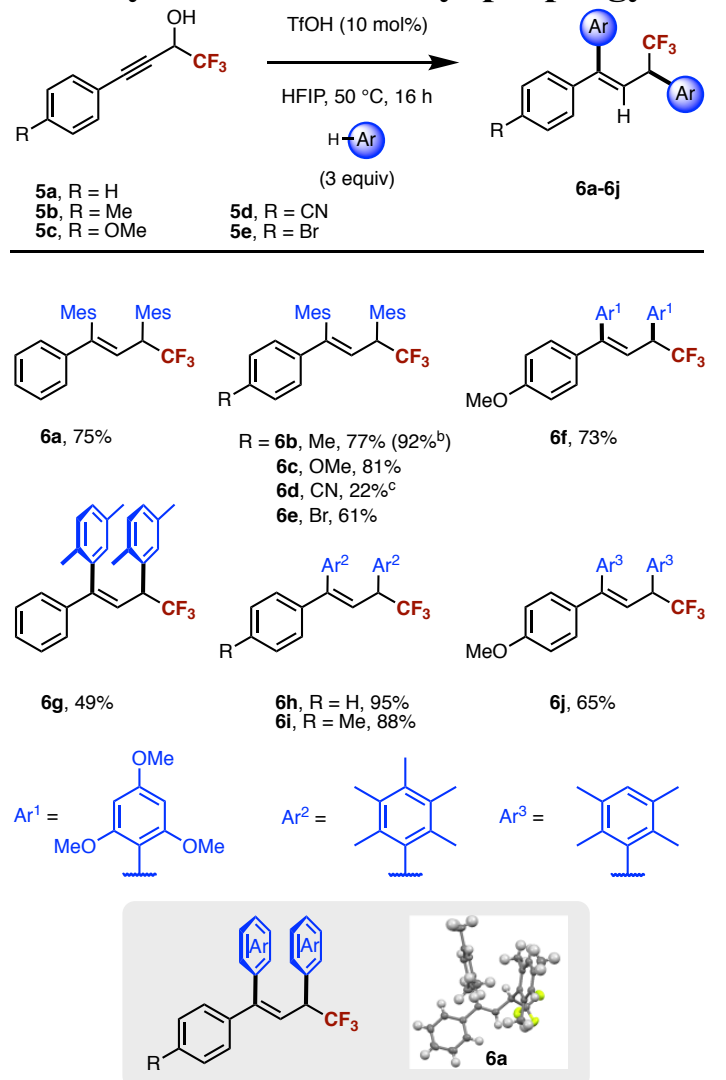

${ }^{a}$ Isolated yields after column chromatography. ${ }^{b}$ Performed at $1 \mathrm{mmol}$ scale.

${ }^{c}$ Reaction heated at $100{ }^{\circ} \mathrm{C}$ for $88 \mathrm{~h}$. Mes = mesityl. 
Mechanistic experiments. To determine whether the $\mathrm{FeCl}_{3}$ acts as a Lewis acid or Brønsted acid catalyst, ${ }^{7}$ reactions were carried out in the presence of catalytic quantities $(10 \mathrm{~mol} \%)$ of the hindered Brønsted base 2,6-di-tert-butylpyridine, which does not coordinate most metal ions, ${ }^{26}$ and in the presence of a similar amount of Proton-sponge (1,8Bis(dimethylamino)naphthalene, $N, N, N^{\prime}, N^{\prime}$-Tetramethyl-1,8-naphthalenediamine). In both cases, no reaction was observed, suggesting that the role of the $\mathrm{FeCl}_{3}$ is to generate a hidden Brønsted acid catalyst. However, substituting $\mathrm{FeCl}_{3}$ with $10 \mathrm{~mol} \% \mathrm{HCl}$ gave only traces of product, excluding $\mathrm{HCl}$ as the active catalyst. Therefore, we suspect that the active catalytic species is a partially hydrated or HFIP-bound ferric ion that acts as a Brønsted acid catalyst, although rapid and complex equilibria between many possible species make this very difficult to determine definitively.

Insight into the mechanism of the cyclization of allenes to indenes can be extracted from the observed scope of substrates (Table 3). When a methyl group is in the ortho position on the phenyl ring A, like in the cases of $\mathbf{1 e}$ and $\mathbf{1} \mathbf{m}$, the formation of the indene is disabled. Para-bromo substitution in phenyl ring A of the starting alcohols also leads to significantly lower cyclization rates of $\mathbf{1 g}$ and 10. To distinguish whether ring A or ring B are involved in the indene-forming cyclization event, deuterated substrate 1- $d_{5}$ was exposed to the standard reaction conditions. Analysis of the ${ }^{1} \mathrm{H}$ and ${ }^{2} \mathrm{H}$ NMR and high resolution MS of the resulting product revealed its structure to be that of $\mathbf{2 a -} d_{4}$ as shown in Scheme 3, consistent with a Nazarov-type cyclization involving ring A rather than one involving ring B (Scheme 4). Similar cyclizations have been reported to be catalyzed by $\mathrm{Yb}(\mathrm{OTf})_{3},{ }^{27} \mathrm{AgOTf}$, and $\mathrm{TfOH} .{ }^{28}$
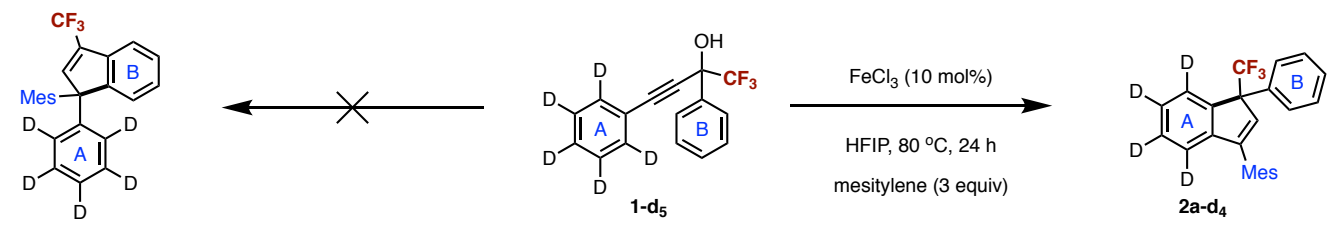

Scheme 3. Reaction of pentadeuterated substrate 1- $d_{5}$ with mesitylene 


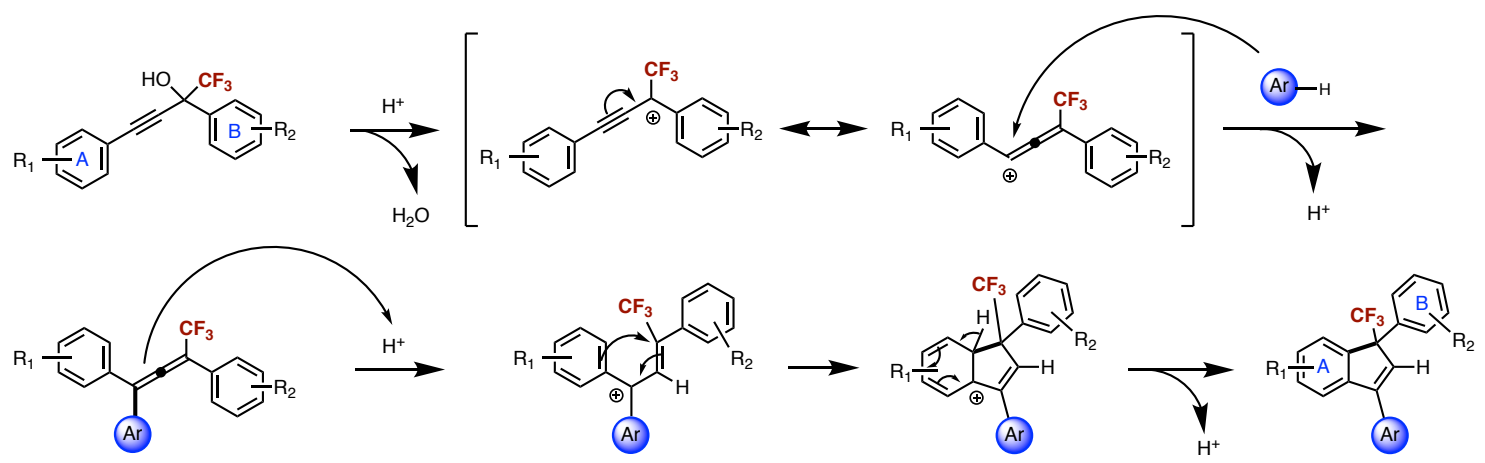

Scheme 4. Proposed mechanism of indene formation

Regarding the bis-addition of arenes to secondary propargylic alcohols (Table 5), the proposed mechanism is shown in Scheme 5. First, the hydroxyl group is activated by the TfOH/HFIP hydrogen-bond network, which is followed by nucleophilic substitution at the $\gamma$-carbon. Then, the $\mathrm{C}-\mathrm{C}$ double bond that is more distant from the $\mathrm{CF}_{3}$ group is protonated, forming a carbocation that is both benzylic and allylic. This carbocation is attacked by a second arene molecule, furnishing a tri-substituted allylic trifluoromethane.

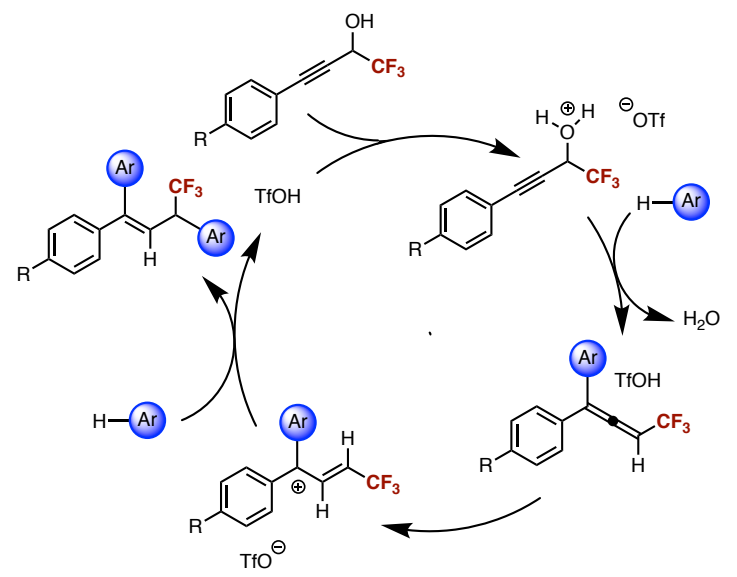

Scheme 5. Plausible mechanism for bis-addition to secondary $\alpha-\mathbf{C F}_{3}$ propargylic alcohols

\section{CONCLUSION}

We have described a straightforward catalytic method to access triaryl $\mathrm{CF}_{3}$ - bearing allenes, indenes, chromenes and 1,1,1-trifluoro-3-butenes directly from $\alpha-\mathrm{CF}_{3}$ propargylic alcohols. The important role of HFIP solvent in combination with strong Brønsted or Lewis acids such as triflic acid or $\mathrm{FeCl}_{3}$ were key to the observed reactivity. Mechanistic experiments suggest the initial 
formation of a $\mathrm{CF}_{3}$-allene intermediate, which subsequently cyclizes to the corresponding $\mathrm{CF}_{3}$ indene.

\section{EXPERIMENTAL SECTION}

General Information. All Friedel-Crafts reactions were performed in $10 \mathrm{~mL}$ glass pressure tubes under an atmosphere of air. Elevated temperatures were achieved by way of a stirrer-hotplate, metal heating block and thermocouple. Purification of reaction products was carried out by flash column chromatography using Merck silica gel (40-63 $\mu \mathrm{m})$. Analytical thin layer chromatography (TLC) was performed on aluminum sheets precoated with silica gel 60 F254 (Merck), cut to size. Visualization was accomplished with UV light. ${ }^{1} \mathrm{H}$ NMR spectra were recorded on a Bruker UltraShield Plus 400 (400 MHz) spectrometer at ambient temperature and are reported in ppm using solvent as internal standard (residual $\mathrm{CHCl}_{3}$ at $7.26 \mathrm{ppm}$ ). ${ }^{13} \mathrm{C}\left\{{ }^{1} \mathrm{H}\right\} \mathrm{NMR}$ spectra were recorded on a Bruker UltraShield Plus 400 (100 MHz) spectrometer at ambient temperature and are reported in ppm using solvent as internal standard $\left(\mathrm{CDCl}_{3}\right.$ at $\left.77.16 \mathrm{ppm}\right) .{ }^{19} \mathrm{~F}$ NMR spectra were recorded on a Bruker UltraShield Plus 400 (376.5 MHz) spectrometer at ambient temperature and are reported in ppm using trifluoroacetic acid as external standard (peak at $-76.55 \mathrm{ppm}$ ). Data are reported as: multiplicity $(\mathrm{ap}=$ apparent, $\mathrm{br}=$ broad, $\mathrm{s}=$ singlet, $\mathrm{d}=$ doublet, $\mathrm{t}=$ triplet, $\mathrm{q}=$ quartet, quint $=$ quintet, sext $=$ sextet, $\mathrm{m}=$ multiplet, $\mathrm{dd}=$ doublet of doublets, $\mathrm{ddd}=$ doublet of doublet of doublets, $\mathrm{dddd}=$ doublet of doublet of doublet of doublets, $\mathrm{qd}=$ quartet of doublets, $\mathrm{dt}$ $=$ doublet of triplets, $\mathrm{dm}=$ doublet of multiplets, $\mathrm{td}=$ triplet of doublets, quintd $=$ quintet of doublets), coupling constants (in $\mathrm{Hz}$ ) and integration. In cases where compounds were isolated as mixtures of regioisomers, signals corresponding to protons of the major regioisomer were integrated as integer values matching the number of protons in the molecule. Non-integer integration values correspond to signals of protons of minor regioisomers or to overlapping signals of regioisomers. GC/MS analysis was conducted on a GC System 7820A (G4320) connected to a MSD block 5977E (G7036A) using Agilent High Resolution Gas Chromatography Column HP5MS UI, $30 \mathrm{~m} \times 0.250 \mathrm{~mm} \times 0.25 \mu \mathrm{m}$. High resolution mass spectrometry (HRMS) analysis was performed on instruments GCT 1er Waters (EI and CI), MicroTOF-Q Bruker (ESI), and a GC Thermo Scientific Trace 1300 GC unit coupled to an APPI MasCom source mounted on a Thermo Scientific Exactive Plus EMR mass unit (Orbitrap FT-HRMS analyzer). Materials: All 
commercial materials were purchased from Sigma-Aldrich, Alfa Aesar and FluoroChem, and were used as received, without further purification.

\section{Preparation of tertiary propargylic alcohols}

General procedure A for tertiary propargylic alcohols synthesis: Trifluoromethyl phenyl ketone (5.0-10 mmol, 1.0 equiv) and phenyl acetylene (1.5 equiv) were diluted in 10-15 mL DMSO. CuI ( 0.10 equiv) and $\mathrm{K}_{2} \mathrm{CO}_{3}$ ( 0.20 equiv) were added and the reaction mixture was heated at $50-70{ }^{\circ} \mathrm{C}$ for $24 \mathrm{~h}$. The reaction mixture was then treated with brine, extracted with $\mathrm{CH}_{2} \mathrm{Cl}_{2}$, dried with anhydrous sodium sulfate and concentrated at reduced pressure. The product was then purified by silica gel column chromatography.

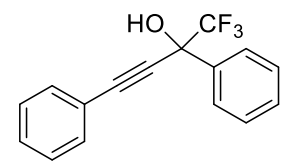

1,1,1-Trifluoro-2,4-diphenylbut-3-yn-2-ol was prepared according to general procedure A and isolated as a yellow oil. Spectral data are in agreement with the literature. ${ }^{29}$ Isolated $2.55 \mathrm{~g}, 85 \%$ yield. ${ }^{1} \mathrm{H}$ NMR (400 $\left.\mathrm{MHz}, \mathrm{CDCl}_{3}\right): \delta(\mathrm{ppm})$ 7.86-7.79 (m, 2H), 7.57-7.52 (m, 2H), 7.48-7.42 (m, 3H), 7.42-7.32 (m, 3H),

$3.10(\mathrm{~s}, 1 \mathrm{H})$.

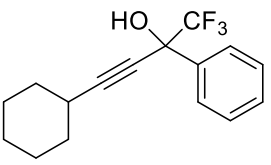

4-Cyclohexyl-1,1,1-trifluoro-2-phenylbut-3yn-2-ol was prepared according to general procedure A and isolated as a yellow oil. Spectral data are in agreement with the literature. ${ }^{30}$ Isolated $1.32 \mathrm{~g}, 44 \%$ yield. ${ }^{1} \mathrm{H} \mathrm{NMR}\left(400 \mathrm{MHz}, \mathrm{CDCl}_{3}\right): \delta$ (ppm) 7.82-7.68 (m, 2H), 7.43-7.39 (m, 3H), 2.92 (s, 1H), 2.54 (sept. $J=4 \mathrm{~Hz}$, 1H), 1.90-1.29 (m, 10H).

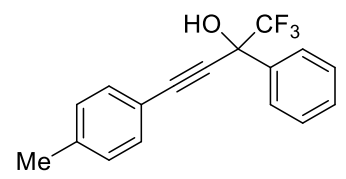

1,1,1-Trifluoro-2-phenyl-4-(p-toluyl)but-3-yn-2-ol was prepared according to general procedure A and isolated as a pale yellow oil. Spectral data are in agreement with the literature. ${ }^{31}$ Isolated $2.55 \mathrm{~g}, 85 \%$ yield. ${ }^{1} \mathrm{H}$ NMR (400 $\left.\mathrm{MHz}, \mathrm{CDCl}_{3}\right): \delta(\mathrm{ppm})$ 7.86-7.78 (m, 2H), 7.52-7.36 (m, 5H), $7.17(\mathrm{~d}, J=$ $7.8 \mathrm{~Hz}, 2 \mathrm{H}), 3.08(\mathrm{~s}, 1 \mathrm{H}), 2.38(\mathrm{~s}, 3 \mathrm{H})$.

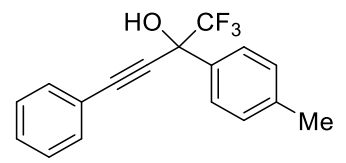

1,1,1-Trifluoro-4-phenyl-2-(p-toluyl)but-3-yn-2-ol was prepared according to general procedure $\mathrm{A}$ and isolated as a yellow oil. Spectral data are in agreement with the literature. ${ }^{32}$ Isolated $0.93 \mathrm{~g}, 31 \%$ yield. ${ }^{1} \mathrm{H}$ NMR (400 $\left.\mathrm{MHz}, \mathrm{CDCl}_{3}\right): \delta(\mathrm{ppm}) 7.62(\mathrm{~d}, J=8.1 \mathrm{~Hz}, 2 \mathrm{H}), 7.48-7.43(\mathrm{~m}, 2 \mathrm{H}), 7.35-$ $7.25(\mathrm{~m}, 3 \mathrm{H}), 7.16(\mathrm{~d}, J=8.7 \mathrm{~Hz}, 2 \mathrm{H}), 2.98(\mathrm{~s}, 1 \mathrm{H}), 2.31$ (s, 3H).

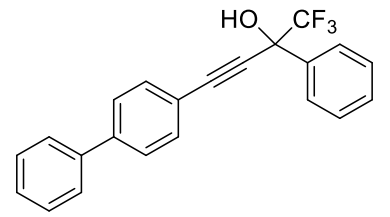

4-((1,1'-Biphenyl)-4-yl)-1,1,1-trifluoro-2-phenylbut-3-yn-2-ol was prepared according to general procedure $A$ and isolated as yellow solid. Isolated $2.01 \mathrm{~g}, 67 \%$ yield. ${ }^{1} \mathrm{H}$ NMR $\left(500 \mathrm{MHz}, \mathrm{CDCl}_{3}\right): \delta(\mathrm{ppm}) 7.86-$ 7.80 (m, 2H), 7.63-7.57 (m, 6H), 7.48-7.42 (m, 4H), 7.38 (t, $J=7.4 \mathrm{~Hz}$, 2H), $3.09(\mathrm{~s}, 1 \mathrm{H}) .{ }^{13} \mathrm{C}\left\{{ }^{1} \mathrm{H}\right\} \mathrm{NMR}\left(100 \mathrm{MHz}, \mathrm{CDCl}_{3}\right): \delta$ (ppm) 142.5, 
$140.2,135.4,132.7,129.7,129.1,128.4,128.1,127.4,127.3,127.2,123.5$ (q, $J=283.9 \mathrm{~Hz}), 119.9$, 88.1, 85.1, 73.5 (q, $J=32.3 \mathrm{~Hz}),{ }^{19} \mathrm{~F}$ NMR (376.5 MHz, $\mathrm{CDCl}_{3}, \mathrm{CF}_{3} \mathrm{COOH}-$ ext. st.): $\delta$ (ppm) 80.9 (s, 3F). HRMS (APPI ${ }^{+}$-Orbitrap) $m / z$ : $\left[\mathrm{M}+\mathrm{H}-\mathrm{H}_{2} \mathrm{O}\right]^{+}$: Calcd for $\mathrm{C}_{22} \mathrm{H}_{15} \mathrm{~F}_{3} \mathrm{O}$ 335.1042; Found 335.1054 (3.5 ppm).

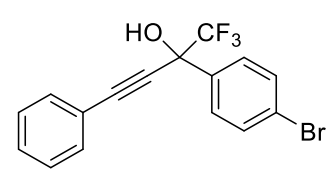

4-(4-Bromophenyl)-1,1,1-trifluoro-2-phenylbut-3-yn-2-ol was prepared according to general procedure $A$ and isolated as a yellow oil. Spectral data are in agreement with the literature. ${ }^{13}$ Isolated $2.70 \mathrm{~g}, 90 \%$ yield. $\mathrm{R}_{\mathrm{f}}=0.43$ (petroleum ether/EtOAc 9:1). ${ }^{1} \mathrm{H}$ NMR $\left(500 \mathrm{MHz}, \mathrm{CDCl}_{3}\right): \delta(\mathrm{ppm}) 7.68(\mathrm{~d}$, $J=8.6 \mathrm{~Hz}, 2 \mathrm{H}), 7.58-7.50(\mathrm{~m}, 4 \mathrm{H}), 7.44-7.35(\mathrm{~m}, 3 \mathrm{H}), 3.26(\mathrm{~s}, 1 \mathrm{H})$.

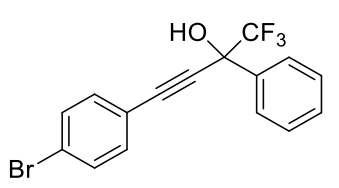

4-(4-Bromophenyl)-1,1,1-trifluoro-2-phenylbut-3-yn-2-ol was prepared according to general procedure A and isolated as a yellow oil. Spectral data are in agreement with the literature. ${ }^{13}$ Isolated $1.56 \mathrm{~g}, 52 \%$ yield. $\mathrm{R}_{\mathrm{f}}=0.44$ (petroleum ether/EtOAc 9:1). ${ }^{1} \mathrm{H}$ NMR (400 $\left.\mathrm{MHz} \mathrm{CDCl}_{3}\right): \delta(\mathrm{ppm}) 7.75-$ 7.69 (m, 2H), 7.46-7.41 (m, 2H), 7.39-7.34 (m, 3H), 7.34-7.28 (m, 2H), 3.09 (s, 1H).

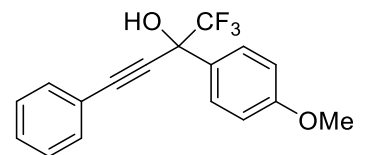

1,1,1-Trifluoro-2-(4-methoxyphenyl)-4-phenylbut-3-yn-2-ol was prepared according to general procedure $A$ and isolated as pale yellow solid. Spectral data are in agreement with the literature. ${ }^{30}$ Isolated $1.92 \mathrm{~g}, 64 \%$ yield. ${ }^{1} \mathrm{H}$ NMR (400 MHz, $\left.\mathrm{CDCl}_{3}\right): \delta(\mathrm{ppm}) 7.73(\mathrm{~d}, J=8.8 \mathrm{~Hz}, 2 \mathrm{H}), 7.57-7.50(\mathrm{~m}$, 2H), 7.43-7.32 (m, 3H), $6.96(\mathrm{~d}, J=8.9 \mathrm{~Hz}, 2 \mathrm{H}), 3.84(\mathrm{~s}, 3 \mathrm{H}), 3.10(\mathrm{~s}, 1 \mathrm{H})$.

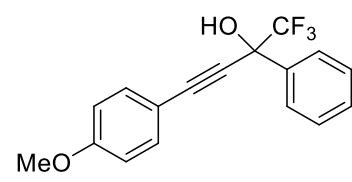

1,1,1-Trifluoro-2-(4-methoxy-phenyl)-4-phenylbut-3-yn-2-ol was prepared according to general procedure A and isolated as a yellow oil. Spectral data are in agreement with the literature. ${ }^{30}$ Isolated $2.73 \mathrm{~g}, 91 \%$ yield. $\mathrm{R}_{\mathrm{f}}=0.36$ (petroleum ether/EtOAc 9:1). ${ }^{1} \mathrm{H}$ NMR (400 MHz, $\left.\mathrm{CDCl}_{3}\right): \delta(\mathrm{ppm}) 7.73$ $(\mathrm{dt}, J=8.8 \mathrm{~Hz}, 3.0 \mathrm{~Hz}, 2 \mathrm{H}), 7.56-7.51(\mathrm{~m}, 2 \mathrm{H}), 7.45-7.33(\mathrm{~m}, 3 \mathrm{H}), 6.93-6.10(\mathrm{~m}, 2 \mathrm{H}), 3.84(\mathrm{~s}$, $3 \mathrm{H}), 3.10(\mathrm{~s}, 1 \mathrm{H})$.

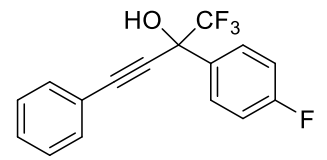

1,1,1-Trifluoro-2-(4-fluorophenyl)-4-phenylbut-3-yn-2-ol was prepared according to general procedure $\mathrm{A}$ and isolated as a yellow oil. Spectral data are in agreement with the literature. ${ }^{13}$ Isolated $2.85 \mathrm{~g}, 95 \%$ yield. $\mathrm{R}_{\mathrm{f}}=0.40$ (petroleum ether/EtOAc 9:1). ${ }^{1} \mathrm{H} \mathrm{NMR}\left(400 \mathrm{MHz}, \mathrm{CDCl}_{3}\right): \delta(\mathrm{ppm})$ 7.83-7.76 (m, 2H), 7.56-7.50 (m, 2H), 7.45-7.34 (m, 3H), 7.16-7.08 (m, 2H), 3.29 (s, 1H).

\section{Preparation of allenes}

General procedure B: To a $10 \mathrm{~mL}$ reaction tube was added the catalyst ( $10 \mathrm{~mol} \%)$, HFIP (0.50 $\mathrm{M}$ relative to propargylic alcohol), propargylic alcohol (0.17-0.40 mmol, 1.0 equiv), followed by the arene nucleophile (5.0 equiv). The mixture was allowed to stir at $25^{\circ} \mathrm{C}$ until judged complete by TLC (9:1 Petroleum ether:EtOAc), typically after $10 \mathrm{~min}$. The reactions typically turn 
an opaque black. The crude reaction mixture was directly transferred for silica gel chromatography.

General procedure C: To a $10 \mathrm{~mL}$ reaction tube was added the catalyst ( $10 \mathrm{~mol} \%)$, HFIP (0.5-1.0 M relative to propargylic alcohol), and propargylic alcohol (0.17-0.40 mmol, 1.0 equiv), followed by the arene nucleophile (5.0 equiv). The reactions typically turn an opaque black. After completion of the reaction as judged by TLC, the crude reaction mixture was directly transferred for silica gel chromatography.

\section{Characterization data for allenes}

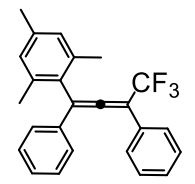

1-Mesityl-1,3-diphenyl-4,4,4-trifluoro-1,2-butadiene (1a) was prepared according to general procedure B from 1,1,1-trifluoro-2,4-diphenylbut-3-yn-2-ol (100 mg, 0.363 $\mathrm{mmol})$ and mesitylene $(253 \mu \mathrm{L}, 1.82 \mathrm{mmol}, 5.0$ equiv $)$ with $5.9 \mathrm{mg}(0.036 \mathrm{mmol})$ of $\mathrm{FeCl}_{3}$ in $0.73 \mathrm{~mL}$ of HFIP. The reaction mixture was stirred at ambient temperature for $10 \mathrm{~min}$. Purification by flash column chromatography over silica (petroleum ether) gave 128 mg $\left(93 \%\right.$ yield) of white solid. $\mathrm{R}_{\mathrm{f}}=0.83$ (petroleum ether/EtOAc 9:1). ${ }^{1} \mathrm{H}$ NMR (400 MHz, $\left.\mathrm{CDCl}_{3}\right): \delta(\mathrm{ppm}) 7.49(\mathrm{~d}, J=7.5 \mathrm{~Hz}, 2 \mathrm{H}), 7.43-7.12(\mathrm{~m}, 8 \mathrm{H}), 6.94(\mathrm{~s}, 2 \mathrm{H}), 2.32(\mathrm{~s}, 3 \mathrm{H}), 2.17(\mathrm{~s}$, $6 \mathrm{H}) .{ }^{13} \mathrm{C}\left\{{ }^{1} \mathrm{H}\right\} \mathrm{NMR}\left(100 \mathrm{MHz}, \mathrm{CDCl}_{3}\right): \delta(\mathrm{ppm}) 205.1$ (q, $\left.J=3.5 \mathrm{~Hz}\right), 138.1,137.1,133.3,130.2$, $129.9,129.2,128.9,128.7,128.6,128.5,127.6,126.7,123.7$ (q, $J=275.1 \mathrm{~Hz}), 114.1,104.0(\mathrm{q}, J$ $=34.3 \mathrm{~Hz}$ ), 21.2, 20.4. ${ }^{19} \mathrm{~F} \mathrm{NMR}\left(376.5 \mathrm{MHz}, \mathrm{CDCl}_{3}, \mathrm{CF}_{3} \mathrm{COOH}\right.$ - ext. st.): $\delta$ (ppm) -62.5 (s, 3F). HRMS (APPI ${ }^{+}$-Orbitrap) $m / z$ : [M] ${ }^{+}$. Calcd for $\mathrm{C}_{25} \mathrm{H}_{21} \mathrm{~F}_{3}$ 378.1590; Found 378.1596 (1.5 ppm).

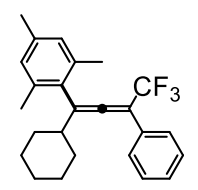

1-Cyclohexyl-1-mesityl-3-phenyl-4,4,4-trifluoro-1,2-butadiene (1b) was prepared according to general procedure $\mathrm{B}$ from 4-cyclohexyl-1,1,1-trifluoro-2-phenylbut-3yn2-ol (49 $\mathrm{mg}, 0.17 \mathrm{mmol})$ and mesitylene $(70 \mu \mathrm{L}, 0.50 \mathrm{mmol}, 3.0$ equiv) with $2.7 \mathrm{mg}$ $(0.017 \mathrm{mmol})$ of $\mathrm{FeCl}_{3}$, in $0.33 \mathrm{~mL}$ of HFIP. The reaction mixture was stirred at 50 ${ }^{\circ} \mathrm{C}$ for $24 \mathrm{~h}$. Purification by flash column chromatography over silica (petroleum ether) gave 33 mg (51\% yield) of the product. ${ }^{1} \mathrm{H}$ NMR (400 $\left.\mathrm{MHz}, \mathrm{CDCl}_{3}\right): \delta(\mathrm{ppm}) 7.48(\mathrm{~d}, J=7.8 \mathrm{~Hz}, 2 \mathrm{H})$, $7.37(\mathrm{t}, J=7.7 \mathrm{~Hz}, 2 \mathrm{H}), 7.30(\mathrm{t}, J=7.2 \mathrm{~Hz}, 1 \mathrm{H}), 6.89(\mathrm{~s}, 2 \mathrm{H}), 2.41-2.15(\mathrm{~m}, 10 \mathrm{H}), 2.03-1.91(\mathrm{~m}$, 2H), 1.87-1.75 (m, 2H), 1.75-1.66 (m, 1H), 1.43-1.18 (m, 5H). ${ }^{13} \mathrm{C}\left\{{ }^{1} \mathrm{H}\right\}$ NMR $\left(100 \mathrm{MHz}, \mathrm{CDCl}_{3}\right)$ : $\delta(\mathrm{ppm}) 201.5(\mathrm{q}, J=4.0 \mathrm{~Hz}), 137.2,131.9,130.9,128.7,128.0,127.32,127.31,124.1(\mathrm{q}, J=$ $272.7 \mathrm{~Hz}), 118.1,102.2$ (q, $J=33.8 \mathrm{~Hz}), 43.4,32.1,31.9,26.7,26.7,26.2,21.1 .{ }^{19} \mathrm{~F} \mathrm{NMR}(376.5$ $\left.\mathrm{MHz}, \mathrm{CDCl}_{3}\right): \delta(\mathrm{ppm})-58.1$ (s, 3F). HRMS (APPI ${ }^{+}$-Orbitrap) $m / z$ : [M] $]^{+}$Calcd for $\mathrm{C}_{25} \mathrm{H}_{27} \mathrm{~F}_{3}$ 384.2059; Found 384.2059 (-0.1 ppm).

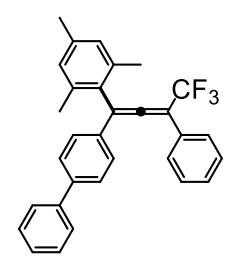

1-Mesityl-1-(1,1'-biphenyl-4-yl)-4,4,4-trifluoro-3-phenyl-1,2-butadiene (1c) was prepared according to general procedure B from 4-((1,1'-biphenyl)-4-yl)-1,1,1trifluoro-2-phenylbut-3-yn-2-ol (59 mg, $0.17 \mathrm{mmol})$ and mesitylene $(71 \mu \mathrm{L}, 0.51$ mmol, 3.0 equiv) with $2.7 \mathrm{mg}(0.017 \mathrm{mmol})$ of $\mathrm{FeCl}_{3}$, in $0.34 \mathrm{~mL}$ of HFIP. The reaction mixture was stirred at ambient temperature for $10 \mathrm{~min}$. Purification by flash column chromatography over silica (petroleum ether) gave $31 \mathrm{mg}$ (40\% yield) of the product with $95 \%$ purity (the rest is the corresponding indene that started to form quickly). ${ }^{1} \mathrm{H} \mathrm{NMR}\left(400 \mathrm{MHz}, \mathrm{CDCl}_{3}\right.$ ): $\delta(\mathrm{ppm}) 7.67-7.56(\mathrm{~m}, 4 \mathrm{H}), 7.54(\mathrm{~d}, J=7.5 \mathrm{~Hz}, 2 \mathrm{H}), 7.46$ (t, $J=7.5$ 
$\mathrm{Hz}, 2 \mathrm{H}), 7.43-7.30(\mathrm{~m}, 6 \mathrm{H}), 6.99(\mathrm{~s}, 2 \mathrm{H}), 2.36(\mathrm{~s}, 3 \mathrm{H}), 2.23(\mathrm{~s}, 6 \mathrm{H}) .{ }^{13} \mathrm{C}\left\{{ }^{1} \mathrm{H}\right\} \mathrm{NMR}(100 \mathrm{MHz}$, $\left.\mathrm{CDCl}_{3}\right): \delta(\mathrm{ppm}) 205.3(\mathrm{q}, J=3.9 \mathrm{~Hz}), 141.5,140.6,138.1,137.2,132.2,130.2,129.9,129.0$, $128.9,128.8,128.6,127.9,127.7,127.7,127.2,127.1,123.7$ (q, $J=273.3 \mathrm{~Hz}), 113.9,104.1(\mathrm{q}, J$ $=34.1 \mathrm{~Hz}), 21.2,20.4 .{ }^{19} \mathrm{~F}$ NMR $\left(376.5 \mathrm{MHz}, \mathrm{CDCl}_{3}\right): \delta(\mathrm{ppm})-58.1(\mathrm{~s}, 3 \mathrm{~F})$. HRMS (APPI ${ }^{+}$ Orbitrap) $m / z$ : $[\mathrm{M}]^{+}$. Calcd for $\mathrm{C}_{31} \mathrm{H}_{25} \mathrm{~F}_{3}$ 454.1903; Found 454.1903 (0.1 ppm).

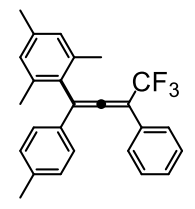

1-Mesityl-4,4,4-trifluoro-1-(p-toluyl)-3-phenyl-1,2-butadiene (1d) was prepared according to general procedure B from 1,1,1-trifluoro-2-phenyl-4-( $p$-tolulyl)but-3yn-2-ol (85 mg, $0.29 \mathrm{mmol})$ and mesitylene $(117 \mu \mathrm{L}, 0.84 \mathrm{mmol}, 5.0$ equiv) with 4.6 $\mathrm{mg}(0.028 \mathrm{mmol})$ of $\mathrm{FeCl}_{3}$, in $0.57 \mathrm{~mL}$ of HFIP. The reaction mixture was stirred at ambient temperature for $10 \mathrm{~min}$. Purification by flash column chromatography over silica (petroleum ether) gave $84 \mathrm{mg}\left(74 \%\right.$ yield) of the product. ${ }^{1} \mathrm{H} \mathrm{NMR}\left(400 \mathrm{MHz}, \mathrm{CDCl}_{3}\right): \delta$ (ppm) $7.63(\mathrm{~d}, J=7.6 \mathrm{~Hz}, 2 \mathrm{H}), 7.50-7.31(\mathrm{~m}, 3 \mathrm{H}), 7.33-7.22(\mathrm{~m}, 4 \mathrm{H}), 7.07(\mathrm{~s}, 2 \mathrm{H}), 2.46(\mathrm{~s}, 3 \mathrm{H})$, $2.44(\mathrm{~s}, 3 \mathrm{H}), 2.32(6 \mathrm{H}) .{ }^{13} \mathrm{C}\left\{{ }^{1} \mathrm{H}\right\} \mathrm{NMR}\left(100 \mathrm{MHz}, \mathrm{CDCl}_{3}\right): \delta(\mathrm{ppm}) 205.0(\mathrm{q}, J=4.0 \mathrm{~Hz}), 138.7$, $138.0,137.1,130.4,130.3,130.0,128.9,128.7,128.5,127.6,127.1,126.1,123.8$ (q, $J=273.5$ $\mathrm{Hz}), 114.1,103.9$ (q, $J=34.2 \mathrm{~Hz}), 21.4,21.2,20.4 .{ }^{19} \mathrm{~F} \mathrm{NMR}\left(376.5 \mathrm{MHz}, \mathrm{CDCl}_{3}\right): \delta(\mathrm{ppm})-59.2$ (s, 3F). HRMS (APPI ${ }^{+}$-Orbitrap) $m / z$ : [M] ${ }^{+}$. Calcd for $\mathrm{C}_{26} \mathrm{H}_{23} \mathrm{~F}_{3} 392.1746$; Found 392.1750 (1.0 ppm).

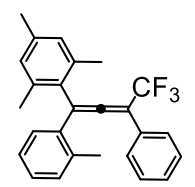

1-Mesityl-3-phenyl-3-(2-toluyl)-4,4,4-trifluoro-1,2-butadiene (1e) was prepared according to general procedure B from 1,1,1-trifluoro-4-phenyl-2-(o-tolulyl)but-3yn-2-ol $(91 \mathrm{mg}, 0.31 \mathrm{mmol})$ and mesitylene $(188 \mu \mathrm{L}, 1.57 \mathrm{mmol}, 5.0$ equiv) with 5.1 $\mathrm{mg}(0.031 \mathrm{mmol})$ of $\mathrm{FeCl}_{3}$, in $0.63 \mathrm{~mL}$ of HFIP. The reaction mixture was stirred at ambient temperature for $10 \mathrm{~min}$. Purification by flash column chromatography over silica (petroleum ether) gave $118 \mathrm{mg}\left(96 \%\right.$ yield) of white solid. $\mathrm{R}_{\mathrm{f}}=0.87$ (petroleum ether/EtOAc 9:1). ${ }^{1} \mathrm{H}$ NMR (400 MHz, $\left.\mathrm{CDCl}_{3}\right): \delta(\mathrm{ppm}) 7.52(\mathrm{~d}, J=7.6 \mathrm{~Hz}, 2 \mathrm{H}), 7.46-7.33(\mathrm{~m}, 3 \mathrm{H}), 7.33-7.23(\mathrm{~m}$, 2H), $7.16(\mathrm{t}, J=7.6 \mathrm{~Hz}, 1 \mathrm{H}), 7.00(\mathrm{~s}, 2 \mathrm{H}), 6.91(\mathrm{~d}, J=7.8 \mathrm{~Hz}, 1 \mathrm{H}), 2.54(\mathrm{~s}, 3 \mathrm{H}), 2.39(\mathrm{~s}, 3 \mathrm{H}), 2.24$ $(\mathrm{s}, 6 \mathrm{H}) .{ }^{13} \mathrm{C}\left\{{ }^{1} \mathrm{H}\right\} \mathrm{NMR}\left(100 \mathrm{MHz}, \mathrm{CDCl}_{3}\right): \delta(\mathrm{ppm}) 205.4(\mathrm{q}, J=4.3 \mathrm{~Hz}), 137.8,137.6,136.9$, $132.6,132.3,131.7,130.7,128.9,128.8,128.4,128.3,128.2,128.0,126.5,123.8$ (q, $J=274.6$ $\mathrm{Hz}), 112.5,101.5$ (q, $J=34.5 \mathrm{~Hz}), 22.0,21.2,20.4 .{ }^{19} \mathrm{~F} \mathrm{NMR}\left(376.5 \mathrm{MHz}, \mathrm{CDCl}_{3}, \mathrm{CF}_{3} \mathrm{COOH}-\right.$ ext. st.): $\delta(\mathrm{ppm})-58.2(\mathrm{~s}, 3 \mathrm{~F})$. HRMS (APPI ${ }^{+}$-Orbitrap) $\mathrm{m} / \mathrm{z}$ : [M] ${ }^{+}$. Calcd for $\mathrm{C}_{26} \mathrm{H}_{23} \mathrm{~F}_{3} 392.1746$; Found 392.1747 (0.2 ppm).

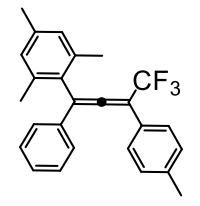

1-Mesityl-1-phenyl-3-toluyl-4,4,4-trifluoro-1,2-butadiene (1f) was prepared according to general procedure B from 1,1,1-trifluoro-4-phenyl-2-( $p$-tolulyl)but-3yn-2-ol (100 mg, $0.346 \mathrm{mmol})$ and mesitylene $(241 \mu \mathrm{L}, 1.73 \mathrm{mmol}, 5.0$ equiv) with $3.5 \mathrm{mg}(0.022 \mathrm{mmol})$ of $\mathrm{FeCl}_{3}$, in $0.69 \mathrm{~mL}$ of HFIP. The reaction mixture was stirred at ambient temperature for $10 \mathrm{~min}$. Purification by flash column chromatography over silica (petroleum ether) gave $122 \mathrm{mg}\left(93 \%\right.$ yield) of a colorless oil. $\mathrm{R}_{\mathrm{f}}=0.93$ (petroleum ether/EtOAc 9:1). ${ }^{1} \mathrm{H}$ NMR (400 MHz, $\left.\mathrm{CDCl}_{3}\right): \delta(\mathrm{ppm}) 7.43(\mathrm{~d}, J=8.0 \mathrm{~Hz}, 2 \mathrm{H}), 7.40-7.32(\mathrm{~m}$, $3 \mathrm{H}), 7.32-7.27(\mathrm{~m}, 2 \mathrm{H}), 7.21(\mathrm{~d}, J=8.0,2 \mathrm{H}), 7.00(\mathrm{~s}, 2 \mathrm{H}), 2.39(\mathrm{~s}, 3 \mathrm{H}), 2.37(\mathrm{~s}, 3 \mathrm{H}), 2.23(\mathrm{~s}, 6 \mathrm{H})$. ${ }^{13} \mathrm{C}\left\{{ }^{1} \mathrm{H}\right\}$ NMR $\left(100 \mathrm{MHz}, \mathrm{CDCl}_{3}\right): \delta(\mathrm{ppm}) 204.8(\mathrm{q}, J=3.8 \mathrm{~Hz}), 138.5,138.0,137.1,133.4$, $129.6,129.1,128.7,128.5,127.5,127.1,127.1,126.7,123.8$ (q, $J=275.2 \mathrm{~Hz}), 113.9,103.8(\mathrm{q}, J$ 
$=34.3 \mathrm{~Hz}$ ), 21.3, 21.2, 20.4. ${ }^{19} \mathrm{~F}$ NMR (376.5 MHz, $\mathrm{CDCl}_{3}, \mathrm{CF}_{3} \mathrm{COOH}$-ext. st.): $\delta$ (ppm) -58.3 (s, 3F). HRMS (APPI ${ }^{+}$-Orbitrap) $m / z$ : [M] ${ }^{+}$. Calcd for $\mathrm{C}_{26} \mathrm{H}_{23} \mathrm{~F}_{3} 392.1746$; Found 392.1744 (-0.6 ppm).

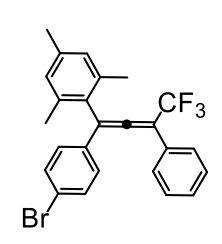

1-(4-Bromophenyl)-1-mesityl-3-phenyl-4,4,4-trifluoro-1,2-butadiene (1g) was prepared according to general procedure B from 4-( $p$-bromophenyl)-1,1,1-trifluoro2-phenyl-but-3-yn-2-ol (86 mg, $0.24 \mathrm{mmol})$ and mesitylene (146 $\mu \mathrm{L}, 1.05 \mathrm{mmol}, 5.0$ equiv) with $3.4 \mathrm{mg}(0.021 \mathrm{mmol})$ of $\mathrm{FeCl}_{3}$, in $0.5 \mathrm{~mL}$ of HFIP. The reaction mixture was stirred at ambient temperature for $10 \mathrm{~min}$. Purification by flash column chromatography over silica (petroleum ether) gave $78 \mathrm{mg}\left(70 \%\right.$ yield) of a colorless oil. ${ }^{1} \mathrm{H} \mathrm{NMR}$ $\left(400 \mathrm{MHz}, \mathrm{CDCl}_{3}\right): \delta(\mathrm{ppm}) 7.52-7.41(\mathrm{~m}, 4 \mathrm{H}), 7.41-7.29(\mathrm{~m}, 3 \mathrm{H}), 7.10(\mathrm{~d}, J=8.6 \mathrm{~Hz}, 2 \mathrm{H}), 6.95$ (s, 2H), $2.32(\mathrm{~s}, 3 \mathrm{H}), 2.15(\mathrm{~s}, 6 \mathrm{H}) .{ }^{13} \mathrm{C}\left\{{ }^{1} \mathrm{H}\right\} \mathrm{NMR}\left(100 \mathrm{MHz}, \mathrm{CDCl}_{3}\right): \delta(\mathrm{ppm}) 204.9(\mathrm{q}, J=3.8$ Hz), 138.3, 137.0, 132.3, 129.8, 129.3, 128.9, 128.8, 128.7, 128.1, 127.6, 127.6, 123.5 (q, $J=273.5$ $\mathrm{Hz}$ ), 122.7, 113.3, 104.3 (q, $J=34.3 \mathrm{~Hz}$ ), 21.1, 20.3. ${ }^{19} \mathrm{~F} \mathrm{NMR} \mathrm{(376.5} \mathrm{MHz,} \mathrm{CDCl}_{3}, \mathrm{C}_{6} \mathrm{~F}_{6}$-ext. st.): $\delta(\mathrm{ppm})-62.5(\mathrm{~s}, 3 \mathrm{~F})$. HRMS (APPI ${ }^{+}$-Orbitrap) $\mathrm{m} / \mathrm{z}$ : $[\mathrm{M}]^{+}$. Calcd for $\mathrm{C}_{25} \mathrm{H}_{20}{ }^{79} \mathrm{BrF}_{3} 456.0695$; Found $456.0700(1.1 \mathrm{ppm})$.

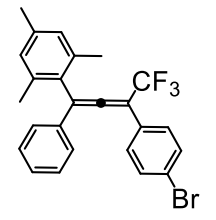

3-(4-Bromophenyl)-1-mesityl-1,3-diphenyl-4,4,4-trifluoro-1,2-butadiene (1h) was prepared according to general procedure B from 4-(4-bromophenyl)-1,1,1-trifluoro2,4-diphenylbut-3-yn-2-ol (100 mg, $0.286 \mathrm{mmol}$ ) and mesitylene (199 $\mu \mathrm{L}, 1.43$ mmol, 5.0 equiv) with $4.3 \mathrm{mg}(0.029 \mathrm{mmol})$ of $\mathrm{FeCl}_{3}$, in $0.57 \mathrm{~mL}$ of HFIP. The reaction mixture was stirred at ambient temperature for $10 \mathrm{~min}$. Purification by flash column chromatography over silica (petroleum ether) gave $118 \mathrm{mg}$ (90\% yield) of a colorless oil. $\mathrm{R}_{\mathrm{f}}=0.82$ (petroleum ether/EtOAc 9:1). ${ }^{1} \mathrm{H}$ NMR (400 MHz, $\left.\mathrm{CDCl}_{3}\right): \delta(\mathrm{ppm}) 7.49(\mathrm{~d}, J=8.6 \mathrm{~Hz}$, 2H), 7.38-7.28 (m, 5H), 7.25-7.18 (m, 2H), $6.95(\mathrm{~s}, 2 \mathrm{H}), 2.33(\mathrm{~s}, 3 \mathrm{H}), 2.16(\mathrm{~s}, 6 \mathrm{H}) .{ }^{13} \mathrm{C}\left\{{ }^{1} \mathrm{H}\right\} \mathrm{NMR}$ $\left(100 \mathrm{MHz}, \mathrm{CDCl}_{3}\right): \delta(\mathrm{ppm}) 205.0(\mathrm{q}, J=3.8 \mathrm{~Hz}), 138.2,137.0,132.9,132.1,129.6,129.3,129.2$, $129.2,128.8,127.1,126.7,123.5(\mathrm{q}, J=275.0 \mathrm{~Hz}), 122.7,114.6,103.2(\mathrm{q}, J=34.6 \mathrm{~Hz}), 21.2$, 20.4. ${ }^{19} \mathrm{~F} \mathrm{NMR}\left(376.5 \mathrm{MHz}, \mathrm{CDCl}_{3}, \mathrm{C}_{6} \mathrm{~F}_{6}\right.$-ext. st.): $\delta$ (ppm) -62.5 (s, 3F). HRMS (APPI ${ }^{+}$-Orbitrap) $\mathrm{m} / \mathrm{z}:[\mathrm{M}]^{+}$. Calcd for $\mathrm{C}_{25} \mathrm{H}_{20}{ }^{79} \mathrm{BrF}_{3}$ 456.0695; Found 456.0699 (0.9 ppm).

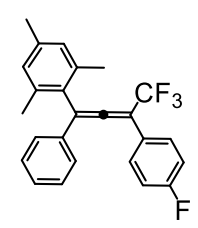

3-(4-Fluorophenyl)-1-mesityl-1-phenyl-4,4,4-trifluoro-1,2-butadiene (1i) was prepared according to modified general procedure B from 1,1,1-trifluoro-2- $(p$ fluorophenyl)-4-phenyl-but-3-yn-2-ol (72 mg, $0.21 \mathrm{mmol})$ and mesitylene (146 $\mu \mathrm{L}$, $1.05 \mathrm{mmol}, 5.0$ equiv) with $3.4 \mathrm{mg}(0.021 \mathrm{mmol})$ of $\mathrm{FeCl}_{3}$, in $0.42 \mathrm{~mL}$ of HFIP. The reaction mixture was stirred at ambient temperature for $1 \mathrm{~h}$. Purification by flash column chromatography over silica (petroleum ether) gave $50 \mathrm{mg}\left(52 \%\right.$ yield) of white solid. ${ }^{1} \mathrm{H}$

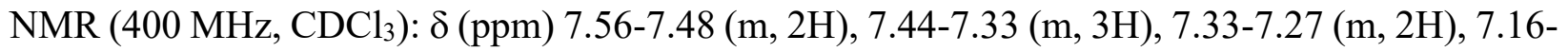
$7.07(\mathrm{~m}, 2 \mathrm{H}), 7.02(\mathrm{~s}, 2 \mathrm{H}), 2.39(\mathrm{~s}, 3 \mathrm{H}), 2.23(\mathrm{~s}, 6 \mathrm{H}) .{ }^{13} \mathrm{C}\left\{{ }^{1} \mathrm{H}\right\} \mathrm{NMR}\left(100 \mathrm{MHz}, \mathrm{CDCl}_{3}\right): \delta(\mathrm{ppm})$ $204.8(\mathrm{q}, J=3.6 \mathrm{~Hz}), 162.9(\mathrm{~d}, J=246.9 \mathrm{~Hz}), 138.2,137.0,133.1,129.7,129.5(\mathrm{~d}, J=8.1 \mathrm{~Hz})$, 129.2, 128.8, 128.7, 126.6, 126.2 (d, $J=3.3 \mathrm{~Hz}), 123.6$ (q, $J=273.3 \mathrm{~Hz}), 116.1$ (d, $J=21.7 \mathrm{~Hz})$, 114.3, 103.1 (q, $J=34.6 \mathrm{~Hz}), 21.3,20.3 .{ }^{19} \mathrm{~F} \mathrm{NMR} \mathrm{(376.5} \mathrm{MHz,} \mathrm{CDCl}_{3}, \mathrm{C}_{6} \mathrm{~F}_{6}$-ext. st.): $\delta$ (ppm) $62.8(\mathrm{~s}, 3 \mathrm{~F}),-116.0(\mathrm{~m}, 1 \mathrm{~F})$. HRMS (APPI ${ }^{+}$-Orbitrap) $\mathrm{m} / \mathrm{z}$ : [M] $]^{+}$. Calcd for $\mathrm{C}_{25} \mathrm{H}_{20} \mathrm{~F}_{4} 396.1496$; Found $396.1500(1.0 \mathrm{ppm})$. 


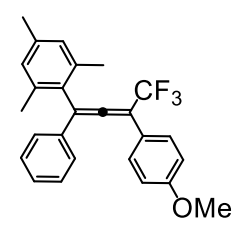

1-Mesityl-3-(4-methoxyphenyl)-1-phenyl-trifluoro-1,2-butadiene (1j) was prepared according to modified general procedure B from 1,1,1-trifluoro-2-(4metoxyphenyl)-4-phenylbut-3-yn-2-ol ol (71 mg, $0.25 \mathrm{mmol})$ and mesitylene (102 $\mu \mathrm{L}, 0.735 \mathrm{mmol}, 3.0$ equiv) with $4.0 \mathrm{mg}(0.025 \mathrm{mmol})$ of $\mathrm{FeCl}_{3}$, in $0.98 \mathrm{~mL}$ of HFIP. The reaction mixture was stirred at ambient temperature for $10 \mathrm{~min}$. Purification by flash column chromatography over silica (petroleum ether/EtOAc 40:1 to 30:1) gave $79 \mathrm{mg}$ (82\% yield) of colorless oil. $\mathrm{R}_{\mathrm{f}}=0.81$ (petroleum ether/EtOAc 9:1). ${ }^{1} \mathrm{H}$ NMR (500 $\left.\mathrm{MHz}, \mathrm{CDCl}_{3}\right): \delta(\mathrm{ppm}) 7.42(\mathrm{~d}, J=8.5 \mathrm{~Hz}, 2 \mathrm{H}), 7.38-7.21(\mathrm{~m}, 5 \mathrm{H}), 6.96(\mathrm{~s}, 2 \mathrm{H}), 6.90(\mathrm{~d}, J=8.8$ $\mathrm{Hz}, 2 \mathrm{H}), 3.82(\mathrm{~s}, 3 \mathrm{H}), 2.34(\mathrm{~s}, 3 \mathrm{H}), 2.18(\mathrm{~s}, 6 \mathrm{H}) .{ }^{13} \mathrm{C}\left\{{ }^{1} \mathrm{H}\right\} \mathrm{NMR}\left(125 \mathrm{MHz}, \mathrm{CDCl}_{3}\right): \delta(\mathrm{ppm}) 204.5$ $(\mathrm{q}, J=3.5 \mathrm{~Hz}), 159.8,138.0,137.1,133.5,130.1,129.1,128.9,128.7,128.5,126.6,123.8(\mathrm{q}, J=$ $275.0 \mathrm{~Hz}), 122.2,114.3,113.8,103.6(\mathrm{q}, J=34.5 \mathrm{~Hz}), 55.4,21.2,20.4 .{ }^{19} \mathrm{~F}$ NMR $(376.5 \mathrm{MHz}$, $\mathrm{CDCl}_{3}, \mathrm{CF}_{3} \mathrm{COOH}$-ext. st.): $\delta$ (ppm) -59.1 (s, 3F). HRMS (APPI -Orbitrap): $m / z[\mathrm{M}]^{+}$. Calcd for $\mathrm{C}_{26} \mathrm{H}_{23} \mathrm{~F}_{3} \mathrm{O}$ 408.1696; Found 408.1708 (2.9 ppm).

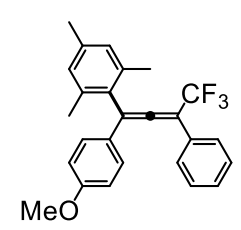

1-Mesityl-4-(4-methoxyphenyl)-3-phenyl-4,4,4-trifluoro-1,2-butadiene (1k) was prepared according to modified general procedure B from 1,1,1-trifluoro-4-(4metoxyphenyl)-2-phenylbut-3-yn-2-ol ol (73 $\mathrm{mg}, 0.24 \mathrm{mmol})$ and mesitylene (84 $\mu \mathrm{L}, 0.74 \mathrm{mmol}, 2.5$ equiv) with $3.9 \mathrm{mg}(0.024 \mathrm{mmol})$ of $\mathrm{FeCl}_{3}$, in $0.48 \mathrm{~mL}$ of HFIP.

The reaction mixture was stirred at ambient temperature for $10 \mathrm{~min}$. Purification by flash column chromatography over silica gave $26 \mathrm{mg}$ (27\% yield) of the product. ${ }^{1} \mathrm{H}$ NMR (400 $\left.\mathrm{MHz}, \mathrm{CDCl}_{3}\right): \delta(\mathrm{ppm}) 7.50(\mathrm{~d}, J=7.6 \mathrm{~Hz}, 2 \mathrm{H}), 7.41-7.29(\mathrm{~m}, 3 \mathrm{H}), 7.20(\mathrm{~d}, J=8.8 \mathrm{~Hz}, 2 \mathrm{H}), 6.95$ $(\mathrm{s}, 2 \mathrm{H}), 6.88(\mathrm{~d}, J=8.8 \mathrm{~Hz}, 2 \mathrm{H}), 3.82(\mathrm{~s}, 3 \mathrm{H}), 2.33(\mathrm{~s}, 3 \mathrm{H}), 2.19(\mathrm{~s}, 6 \mathrm{H}) .{ }^{13} \mathrm{C}\left\{{ }^{1} \mathrm{H}\right\} \mathrm{NMR}(100 \mathrm{MHz}$, $\left.\mathrm{CDCl}_{3}\right): \delta(\mathrm{ppm}) 204.8(\mathrm{q}, J=3.9 \mathrm{~Hz}), 160.0,137.9,137.1,130.5,130.1,128.8,128.7,128.4$, $128.0,127.6,127.6,125.3,123.7(\mathrm{q}, J=273.3 \mathrm{~Hz}), 114.6,113.7,103.8(\mathrm{q}, J=34.1 \mathrm{~Hz}), 55.5$, 21.2, 20.3. ${ }^{19} \mathrm{~F}$ NMR $\left(376.5 \mathrm{MHz}, \mathrm{CDCl}_{3}\right.$ ): $\delta(\mathrm{ppm})-59.3$ (s, 3F). HRMS (APPI $\left.{ }^{+}-O r b i t r a p\right) ~ m / z$ : $[\mathrm{M}+\mathrm{H}]^{+}$. calculated for $\mathrm{C}_{26} \mathrm{H}_{24} \mathrm{~F}_{3} \mathrm{O}$ 409.1774; found 409.1768 (-1.5 ppm).

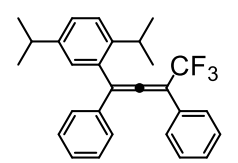

1-(2,5-Diisopropylphenyl)-1,3-diphenyl-4,4,4-trifluoro-1,2-butadiene (11) was prepared according to general procedure B from 1,1,1-trifluoro-2,4-diphenylbut-3yn-2-ol (104 mg, $0.376 \mathrm{mmol})$ and diizopropyl benzene $(356 \mu \mathrm{L}, 1.88 \mathrm{mmol}, 5.0$ equiv) with $6.1 \mathrm{mg}(0.038 \mathrm{mmol})$ of $\mathrm{FeCl}_{3}$, in $0.75 \mathrm{~mL}$ of HFIP. The reaction mixture was stirred at ambient temperature for $10 \mathrm{~min}$. Purification by flash column chromatography over silica (petroleum ether) gave $103 \mathrm{mg}\left(65 \%\right.$ yield) of a colorless oil. $\mathrm{R}_{\mathrm{f}}=0.82$ (petroleum ether/EtOAc 9:1). ${ }^{1} \mathrm{H}$ NMR $\left(400 \mathrm{MHz}, \mathrm{CDCl}_{3}\right)$ : $\delta(\mathrm{ppm}) 7.45(\mathrm{~d}, J=7.9 \mathrm{~Hz}, 2 \mathrm{H}), 7.35-$ $7.27(\mathrm{~m}, 2 \mathrm{H}), 7.25-7.14(\mathrm{~m}, 8 \mathrm{H}), 7.20-7.12(\mathrm{~m}, 1 \mathrm{H}), 2.94$ (sept, $J=6.8 \mathrm{~Hz}, 1 \mathrm{H}), 2.83$ (sept, $J=$ $6.9 \mathrm{~Hz}, 1 \mathrm{H}), 1.18(\mathrm{~d}, J=6.9 \mathrm{~Hz}, 6 \mathrm{H}), 1.04(\mathrm{~d}, J=6.8 \mathrm{~Hz}, 3 \mathrm{H}), 0.94(\mathrm{~d}, J=6.9 \mathrm{~Hz}, 3 \mathrm{H}) .{ }^{13} \mathrm{C}\left\{{ }^{1} \mathrm{H}\right\}$ $\operatorname{NMR}\left(125 \mathrm{MHz}, \mathrm{CDCl}_{3}\right): \delta(\mathrm{ppm}) 205.5$ (q, $\left.J=3.6 \mathrm{~Hz}\right), 146.7,145.0,134.7,132.0,130.1,128.9$, $128.9,128.5,128.5,128.3,127.3,127.3,127.2,126.2,123.6$ (q, $J=273.6 \mathrm{~Hz}), 116.4,103.9$ (q, $J$ $=34.1 \mathrm{~Hz}$ ), 33.7, 30.4, 24.4, 24.1, 24.1, 24.1. ${ }^{19} \mathrm{~F} \mathrm{NMR}$ (376.5 MHz, $\mathrm{CDCl}_{3}, \mathrm{CF}_{3} \mathrm{COOH}$-ext. st.): $\delta(\mathrm{ppm})-58.7$ (s, 3F). HRMS (APPI ${ }^{+}$-Orbitrap) $m / z[\mathrm{M}]^{+}$. Calcd for $\mathrm{C}_{28} \mathrm{H}_{27} \mathrm{~F}_{3}$ 420.2059; Found $420.2062(0.7 \mathrm{ppm})$. 


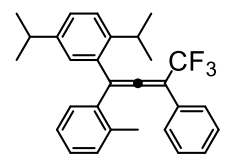

1-(2,5-Diisopropylphenyl)-3-phenyl-3-(2-toluyl)-4,4,4-trifluoro-1,2-butadiene (1m) was prepared according to modified general procedure B from 1,1,1-trifluoro4-phenyl-2-(o-tolulyl)but-3-yn-2-ol (63 $\mathrm{mg}, 0.22 \mathrm{mmol})$ and diisopropylbenzene (123 $\mu \mathrm{L}, 0.647 \mathrm{mmol}, 3.0$ equiv) with $3.5 \mathrm{mg}(0.022 \mathrm{mmol})$ of $\mathrm{FeCl}_{3}$, in $0.86 \mathrm{~mL}$ of HFIP. The reaction mixture was stirred at ambient temperature for $3 \mathrm{~h}$. Purification by flash column chromatography over silica (petroleum ether) gave $63 \mathrm{mg}$ (58\% yield) of colorless oil. $\mathrm{R}_{\mathrm{f}}$ $=0.84$ (petroleum ether/EtOAc 9:1). ${ }^{1} \mathrm{H}$ NMR $\left(500 \mathrm{MHz}, \mathrm{CDCl}_{3}\right): \delta(\mathrm{ppm}) 7.49(\mathrm{~d}, J=7.7 \mathrm{~Hz}, 2 \mathrm{H})$, 7.41-7.34 (m, 2H), 7.34-7.28 (m, 2H), 7.23 (d, $J=2.0 \mathrm{~Hz}, 1 \mathrm{H}), 7.21$ (d, $J=4.0 \mathrm{~Hz}, 2 \mathrm{H}) 7.18-$ $7.13(\mathrm{~m}, 1 \mathrm{H}), 7.11(\mathrm{~d}, J=1.7 \mathrm{~Hz}, 1 \mathrm{H}), 7.03(\mathrm{~d}, J=7.6 \mathrm{~Hz}, 1 \mathrm{H}), 3.00$ (sept, $J=6.8 \mathrm{~Hz}, 1 \mathrm{H}), 2.87$ (sept, $J=6.9 \mathrm{~Hz}, 1 \mathrm{H}) 2.30$ (s, 3H), 1.30-1.16 (d, $J=7.0 \mathrm{~Hz}, 6 \mathrm{H}), 1.04$ (d, $J=6.9 \mathrm{~Hz}, 3 \mathrm{H}), 1.02$ $(\mathrm{d}, J=6.9 \mathrm{~Hz}, 3 \mathrm{H}) .{ }^{13} \mathrm{C}\left\{{ }^{1} \mathrm{H}\right\} \mathrm{NMR}\left(125 \mathrm{MHz}, \mathrm{CDCl}_{3}\right): \delta(\mathrm{ppm}) 205.7$ (q, $\left.J=3.6 \mathrm{~Hz}\right), 146.6,144.8$, 137.0, 134.7, 134.0, 131.3, 130.6, 129.7, 128.8, 128.3, 128.3, 128.2, 127.4 (q, $J=1.1 \mathrm{~Hz}), 127.0$, $126.3,126.2,123.8$ (q, $J=274.8 \mathrm{~Hz}), 115.2,101.4(\mathrm{q}, J=34.1 \mathrm{~Hz}), 33.6,30.0,24.2,24.1,21.5$. ${ }^{19} \mathrm{~F}$ NMR (282 MHz, $\mathrm{CDCl}_{3}, \mathrm{CF}_{3} \mathrm{COOH}$-ext. st.): $\delta$ (ppm) -60.5 (s, 3F). HRMS (APPI ${ }^{+}$-Obitrap) $\mathrm{m} / \mathrm{z}$ : $[\mathrm{M}]^{+}$. Calcd for $\mathrm{C}_{29} \mathrm{H}_{29} \mathrm{~F}_{3}$ 434.2216; Found 434.2226 (2.3 ppm).

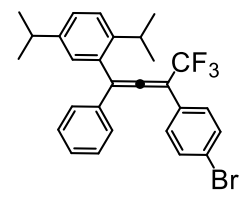

1-(4-Bromophenyl)-1-(2,5-diisopropylphenyl)-3-phenyl-4,4,4-trifluoro-1,2butadiene (1n) was prepared according to general procedure B from 4-(4bromophenyl)-1,1,1-trifluoro-2,4-diphenylbut-3-yn-2-ol (10.2 mg, $0.28 \mathrm{mmol})$ and diisopropylbenzene $(267 \mu \mathrm{L}, 1.411 \mathrm{mmol}, 5.0$ equiv) with $4.5 \mathrm{mg}(0.028$ $\mathrm{mmol})$ of $\mathrm{FeCl}_{3}$, in $0.56 \mathrm{~mL}$ of HFIP. The reaction mixture was stirred at ambient temperature for $10 \mathrm{~min}$. Purification by flash column chromatography over silica (petroleum ether) gave $56 \mathrm{mg}$ ( $40 \%$ yield) of yellow solid. $\mathrm{R}_{\mathrm{f}}=0.87$ (petroleum ether/EtOAc 9:1). ${ }^{1} \mathrm{H}$ NMR (400 $\left.\mathrm{MHz}, \mathrm{CDCl}_{3}\right): \delta(\mathrm{ppm})$ 7.55-7.47 (m, 2H), 7.44-7.28 (m, 7H), 7.25-7.19 (m, 2H), 7.14-7.08 (m, 1H), 2.98 (sept, $J=6.8 \mathrm{~Hz}, 1 \mathrm{H}$ ), 2.90 (sept, $J=6.9 \mathrm{~Hz}, 1 \mathrm{H}$ ), 1.25 (d, $J=6.9 \mathrm{~Hz}, 6 \mathrm{H}), 1.12$ (d, $J=$ $6.8 \mathrm{~Hz}, 3 \mathrm{H}), 1.00(\mathrm{~d}, J=6.8 \mathrm{~Hz}, 3 \mathrm{H}) .{ }^{13} \mathrm{C}\left\{{ }^{1} \mathrm{H}\right\} \mathrm{NMR}\left(125 \mathrm{MHz}, \mathrm{CDCl}_{3}\right): \delta(\mathrm{ppm}) 205.4$ (q, $J=$ $4.2 \mathrm{~Hz}), 146.8,145.0,134.3,132.1,131.7,129.1,129.0,128.8,128.7,128.2$, 127.4, 127.3, 126.3, $123.3(\mathrm{q}, J=275.0 \mathrm{~Hz}), 122.7,117.0,103.1$ (q, $J=34.8 \mathrm{~Hz}), 33.7,30.4,24.4,24.1 .{ }^{19} \mathrm{~F}$ NMR (376.5 MHz, $\mathrm{CDCl}_{3}, \mathrm{CF}_{3} \mathrm{COOH}$-ext. st.): $\delta$ (ppm) -58.8 (s, 3F). HRMS (APPI -Orbitrap) $\mathrm{m} / z$ : $[\mathrm{M}]^{+}$. Calcd for $\mathrm{C}_{28} \mathrm{H}_{26}{ }^{79} \mathrm{BrF}_{3} 498.1165$; Found 498.1168 (0.6 ppm).

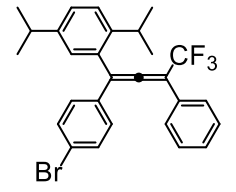

1-(4-Bromophenyl)-1-(2,5-diisopropylphenyl)-3-phenyl-4,4,4-trifluoro-1,2butadiene (10) was prepared according to general procedure B from 4-(4bromophenyl)-1,1,1-trifluoro-2-phenylbut-3-yn-2-ol (111 mg, $0.314 \mathrm{mmol})$ and diisopropylbenzene ( $297 \mu \mathrm{L}, 1.568 \mathrm{mmol}, 5.0$ equiv) with $4.7 \mathrm{mg}(0.031 \mathrm{mmol})$ of $\mathrm{FeCl}_{3}$, in $0.63 \mathrm{~mL}$ of HFIP. The reaction mixture was stirred at ambient temperature for $3 \mathrm{~h}$. Purification by flash column chromatography over silica (petroleum ether) gave $111 \mathrm{mg}(70 \%$ yield) of a colorless oil. $\mathrm{R}_{\mathrm{f}}=0.88$ (petroleum ether/EtOAc 9:1). ${ }^{1} \mathrm{H}$ NMR (500 MHz, $\left.\mathrm{CDCl}_{3}\right)$ : $\delta$ (ppm) $7.52(\mathrm{~d}, J=7.7 \mathrm{~Hz}, 2 \mathrm{H}), 7.47(\mathrm{~d}, J=8.6 \mathrm{~Hz}, 2 \mathrm{H}), 7.42-7.36(\mathrm{~m}, 2 \mathrm{H}), 7.36-7.31(\mathrm{~m}, 2 \mathrm{H})$, 7.31-7.27 (m, 1H), 7.15-7.10 (m, 3H), 2.98 (sept, $J=6.9 \mathrm{~Hz}, 1 \mathrm{H}$ ), 2.92 (sept, $J=6.9 \mathrm{~Hz}, 1 \mathrm{H}$ ), $1.26(\mathrm{~d}, J=6.9 \mathrm{~Hz}, 6 \mathrm{H}), 1.13(\mathrm{~d}, J=6.8 \mathrm{~Hz}, 3 \mathrm{H}), 1.03(\mathrm{~d}, J=6.9 \mathrm{~Hz}, 3 \mathrm{H}) .{ }^{13} \mathrm{C}\left\{{ }^{1} \mathrm{H}\right\}$ NMR $(125$ $\left.\mathrm{MHz}, \mathrm{CDCl}_{3}\right): \delta(\mathrm{ppm}) 206.3(\mathrm{q}, J=3.9 \mathrm{~Hz}), 146.9,145.0,133.8,132.1,131.6,129.7,129.0$, $128.8,128.7,128.2,127.5,127.3,126.3,123.4$ (q, $J=273.5 \mathrm{~Hz}), 122.7,115.7,104.3$ (q, $J=34.2$ 
$\mathrm{Hz}$ ), 33.7, 30.4, 24.4, 24.1, 24.1, 24.1. ${ }^{19} \mathrm{~F}$ NMR (282 MHz, $\mathrm{CDCl}_{3}, \mathrm{CF}_{3} \mathrm{COOH}$-ext. st.): $\delta$ (ppm) -60.8 (s, 3F). HRMS (APPI -Orbitrap) $m / z$ : [M] ${ }^{+}$. Calcd for $\mathrm{C}_{28} \mathrm{H}_{26}{ }^{79} \mathrm{BrF}_{3}$ 498.1165; Found $498.1175(2.0 \mathrm{ppm})$.

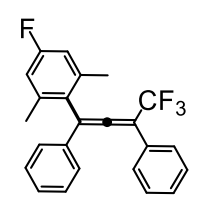

1-(2-(5-Fluoro-m-xylenyl))-1,3-diphenyl-4,4,4-trifluoro-1,2-butadiene (1p) was prepared according to modified general procedure B from 1,1,1-trifluoro-2,4diphenylbut-3-yn-2-ol (104 mg, $0.376 \mathrm{mmol})$ and 5-fluoro- $m$-xylene $(142 \mu \mathrm{L}, 1.13$ mmol, 3.0 equiv) with $6.1 \mathrm{mg}(0.038 \mathrm{mmol})$ of $\mathrm{FeCl}_{3}$, in $1.50 \mathrm{~mL}$ of HFIP. The reaction mixture was heated at $80^{\circ} \mathrm{C}$ for $24 \mathrm{~h}$. Purification by flash column chromatography over silica (petroleum ether) gave $37 \mathrm{mg}$ ( $25 \%$ yield) of colorless oil. $\mathrm{R}_{\mathrm{f}}=0.84$ (petroleum ether/EtOAc 9:1). ${ }^{1} \mathrm{H}$ NMR (500 MHz, $\left.\mathrm{CDCl}_{3}\right): \delta(\mathrm{ppm}) 7.48(\mathrm{~d}, J=7.4 \mathrm{~Hz}, 2 \mathrm{H}), 7.40-7.29(\mathrm{~m}, 6 \mathrm{H}), 7.25-7.20$ (m, 2H), $6.84(\mathrm{~d}, J=9.4 \mathrm{~Hz}, 2 \mathrm{H}), 2.20(\mathrm{~s}, 6 \mathrm{H}) .{ }^{13} \mathrm{C}\left\{{ }^{1} \mathrm{H}\right\} \mathrm{NMR}\left(125 \mathrm{MHz}, \mathrm{CDCl}_{3}\right): \delta(\mathrm{ppm}) 205.1$ (q, $J=3.8 \mathrm{~Hz}), 162.4$ (d, $J=244.7 \mathrm{~Hz}), 139.7$ (d, $J=7.2 \mathrm{~Hz}), 132.9,129.9,129.3,129.0,128.8$, $128.7,128.6(\mathrm{~d}, J=2.0 \mathrm{~Hz}), 127.6(\mathrm{q}, J=1.1 \mathrm{~Hz}), 126.6,123.6(\mathrm{q}, J=273.5 \mathrm{~Hz}), 114.6(\mathrm{~d}, J=$ $21.0 \mathrm{~Hz}), 113.5,104.3$ (q, $J=34.3 \mathrm{~Hz}), 20.6 .{ }^{19} \mathrm{~F} \mathrm{NMR}\left(282 \mathrm{MHz}, \mathrm{CDCl}_{3}, \mathrm{CF}_{3} \mathrm{COOH}\right.$-ext. st.): $\delta$ (ppm) $-60.1(\mathrm{~s}, 3 \mathrm{~F}),-115.9(\mathrm{~s}, 1 \mathrm{H})$. HRMS (APPI ${ }^{+}$-Orbitrap) $\mathrm{m} / \mathrm{z}$ : $[\mathrm{M}]^{+}$. Calcd for $\mathrm{C}_{24} \mathrm{H}_{18} \mathrm{~F}_{4}$ 382.1339; Found 382.1348 (2.4 ppm).

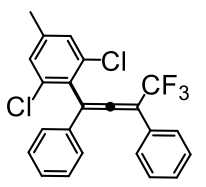

(1-(2,6-Dichloro-4-methylphenyl)-1,3-diphenyl-4,4,4-trifluoro-1,2-butadiene (1q) was prepared according to modified general procedure B from 1,1,1-trifluoro-2,4diphenylbut-3-yn-2-ol (108 mg, $0.392 \mathrm{mmol}$ ) and 3,5-dichlorotoluene (190 mg, 1.18 mmol, 3.0 equiv) with $6.4 \mathrm{mg}(0.039 \mathrm{mmol})$ of $\mathrm{FeCl}_{3}$, in $1.57 \mathrm{~mL}$ of HFIP. The reaction mixture was heated at $80^{\circ} \mathrm{C}$ for $24 \mathrm{~h}$. Purification by flash column chromatography over silica (petroleum ether) gave $31 \mathrm{mg}$ ( $23 \%$ yield) of white solid. ${ }^{1} \mathrm{H}$ NMR $\left(500 \mathrm{MHz}, \mathrm{CDCl}_{3}\right): \delta$ (ppm) $7.56(\mathrm{~d}, J=7.1 \mathrm{~Hz}, 2 \mathrm{H}), 7.41-7.30(\mathrm{~m}, 7 \mathrm{H}), 7.24-7.19(\mathrm{~m}, 3 \mathrm{H}), 2.27(\mathrm{~s}, 3 \mathrm{H}) .7 .56(\mathrm{~d}, J=$ 7.1 Hz, 2H), 7.41-7.30 (m, 7H), 7.24-7.19 (m, 3H), 2.27 (s, 3H). ${ }^{13} \mathrm{C}\left\{{ }^{1} \mathrm{H}\right\}$ NMR (125 MHz, $\left.\mathrm{CDCl}_{3}\right): \delta(\mathrm{ppm}) 205.3(\mathrm{q}, J=3.9 \mathrm{~Hz}), 141.1,135.3,134.7,132.1,131.0,129.3,129.1,129.0$, $128.9,127.7(\mathrm{q}, J=1.2 \mathrm{~Hz}), 127.3,126.5,123.4$ (q, $J=273.9 \mathrm{~Hz}), 112.8,106.6$ (q, $J=34.2 \mathrm{~Hz})$, 20.6. ${ }^{19} \mathrm{~F}$ NMR (282 MHz, $\mathrm{CDCl}_{3}, \mathrm{CF}_{3} \mathrm{COOH}$-ext. st.): $\delta$ (ppm) -60. (s, 3F). HRMS (APPI ${ }^{+}$ Orbitrap) $\mathrm{m} / \mathrm{z}$ : [M] $]^{+}$. Calcd for $\mathrm{C}_{23} \mathrm{H}_{15} \mathrm{Cl}_{2} \mathrm{~F}_{3}$ 418.0497; Found 418.0500 (0.7 ppm).

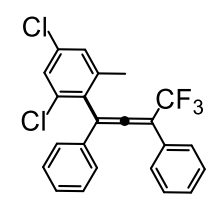

(1-(2,4-Dichloro-5-methylphenyl)-1,3-diphenyl-4,4,4-trifluoro-1,2-butadiene (1q') was prepared according to modified general procedure B from 1,1,1-trifluoro-2,4diphenylbut-3-yn-2-ol (108 mg, $0.392 \mathrm{mmol}$ ) and 3,5-dichlorotoluene (190 mg, 1.18 mmol, 3.0 equiv) with $6.4 \mathrm{mg}(0.039 \mathrm{mmol})$ of $\mathrm{FeCl}_{3}$, in $1.57 \mathrm{~mL}$ of HFIP. The reaction mixture was heated at $80^{\circ} \mathrm{C}$ for $24 \mathrm{~h}$. Purification by flash column chromatography over silica (petroleum ether) gave $17 \mathrm{mg}$ (10\% yield) of white solid ${ }^{1} \mathrm{H} \mathrm{NMR}(500 \mathrm{MHz}, \mathrm{CDCl} 3) \delta(\mathrm{ppm})$ $7.57(\mathrm{~d}, \mathrm{~J}=7.1 \mathrm{~Hz}, 2 \mathrm{H}), 7.43-7.31(\mathrm{~m}, 7 \mathrm{H}), 7.25-7.20(\mathrm{~m}, 3 \mathrm{H}), 2.27(\mathrm{~s}, 3 \mathrm{H}) .{ }^{13} \mathrm{C}\left\{{ }^{1} \mathrm{H}\right\} \mathrm{NMR}$ $\left(125 \mathrm{MHz}, \mathrm{CDCl}_{3}\right): \delta(\mathrm{ppm}) 205.3$ (q, $\left.J=3.9 \mathrm{~Hz}\right), 141.1,135.3,134.7,132.1,131.0,129.3,129.1$, 129.0, 128.9, $127.7(\mathrm{q}, J=1.2 \mathrm{~Hz}), 127.3,126.5,123.4$ (q, $J=273.9 \mathrm{~Hz}), 112.8,106.6$ (q, $J=34.2$ $\mathrm{Hz}$ ), 20.6. ${ }^{19} \mathrm{~F} \mathrm{NMR}\left(282 \mathrm{MHz}, \mathrm{CDCl}_{3}, \mathrm{CF}_{3} \mathrm{COOH}\right.$-ext. st.): $\delta$ (ppm) -60.8 (s, 3F). HRMS (APPI ${ }^{+}$ Orbitrap) $\mathrm{m} / z[\mathrm{M}]^{+}$. Calcd for $\mathrm{C}_{23} \mathrm{H}_{15} \mathrm{Cl}_{2} \mathrm{~F}_{3} 418.0497$; Found 418.0500 (0.7 ppm). 


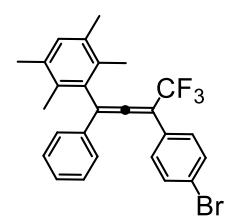

3-(4-Bromophenyl)-1-durenyl-1,3-diphenyl-4,4,4-trifluoro-1,2-butadiene (1s) was prepared according to modified general procedure B 4-(4-bromophenyl)-1,1,1trifluoro-2,4-diphenylbut-3-yn-2-ol (101 mg, $0.284 \mathrm{mmol})$ and durene (114 mg, $0.85 \mathrm{mmol}, 3.0$ equiv. $)$ with $2.9 \mathrm{mg}(0.028 \mathrm{mmol})$ of $\mathrm{FeCl}_{3}$, in $1.13 \mathrm{~mL}$ of HFIP.

The reaction mixture was heated at $80{ }^{\circ} \mathrm{C}$ for $3 \mathrm{~h}$. Purification by flash column chromatography over silica (petroleum ether) gave $125 \mathrm{mg}\left(93 \%\right.$ yield) of white solid. $\mathrm{R}_{\mathrm{f}}=0,90$ (petroleum ether/EtOAc 9:1). ${ }^{1} \mathrm{H}$ NMR (400 MHz, $\left.\mathrm{CDCl}_{3}\right): \delta(\mathrm{ppm}) 7.49$ (dd, $\left.J=8.8,2.0 \mathrm{~Hz}, 2 \mathrm{H}\right)$, 7.39-7.30 (m, 5H), 7.24-7.17 (m, 2H), $7.03(\mathrm{~s}, 1 \mathrm{H}), 2.26(\mathrm{~s}, 3 \mathrm{H}), 2.24(\mathrm{~s}, 3 \mathrm{H}), 2.14(\mathrm{~s}, 3 \mathrm{H}), 2.06$ $(\mathrm{s}, 3 \mathrm{H}) .{ }^{13} \mathrm{C}\left\{{ }^{1} \mathrm{H}\right\}$ NMR $\left(100 \mathrm{MHz}, \mathrm{CDCl}_{3}\right): \delta(\mathrm{ppm}) 205.0(\mathrm{q}, J=3.6 \mathrm{~Hz}), 134.6,134.2,133.2$, 132.6, 132.5, 132.1, 131.7, 129.4, 129.2, 128.8, 126.9, 123.6 (q, $J=275.2 \mathrm{~Hz}), 122.7,115.8,103.2$ (q, $J=34.8 \mathrm{~Hz}$ ), 20.2 (2C), 17.5, 16.7. ${ }^{19} \mathrm{~F} \mathrm{NMR} \mathrm{(376.5} \mathrm{MHz,} \mathrm{CDCl}_{3}, \mathrm{CF}_{3} \mathrm{COOH}$-ext. st.): $\delta$ (ppm) -59.0 (s, 3F). HRMS (APPI ${ }^{+}$-Orbitrap) $\mathrm{m} / z$ : [M] ${ }^{+}$. Calcd for $\mathrm{C}_{26} \mathrm{H}_{22}{ }^{79} \mathrm{BrF}_{3}$ 470.0851; Found $470.0862(2.3 \mathrm{ppm})$.

\section{Characterization data for indenes}

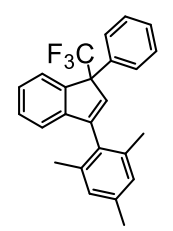

3-Mesityl-1-phenyl-1-(trifluoro-methyl)-1H-indene (2a) was prepared according to general procedure $C$ from 1,1,1-trifluoro-2,4-diphenylbut-3-yn-2-ol (106 mg, 0.382 $\mathrm{mmol})$ and mesitylene $(160 \mu \mathrm{L}, 1.15 \mathrm{mmol}, 3.0$ equiv $)$ with $6.2 \mathrm{mg}(0.038 \mathrm{mmol})$ of $\mathrm{FeCl}_{3}$, in $1.50 \mathrm{~mL}$ of HFIP. The reaction mixture was heated at $80{ }^{\circ} \mathrm{C}$ for $24 \mathrm{~h}$. Purification by flash column chromatography over silica (petroleum ether) gave 137 $\mathrm{mg}\left(94 \%\right.$ yield) of a colorless oil. $\mathrm{R}_{\mathrm{f}}=0.80$ (petroleum ether/EtOAc 9:1). ${ }^{1} \mathrm{H}$ NMR $(400 \mathrm{MHz}$, $\left.\mathrm{CDCl}_{3}\right): \delta(\mathrm{ppm}) 7.68-7.61(\mathrm{~m}, 1 \mathrm{H}), 7.61-7.55(\mathrm{~m}, 2 \mathrm{H}), 7.38-7.29(\mathrm{~m}, 5 \mathrm{H}), 6.98(\mathrm{~s}, 1 \mathrm{H}), 6.94(\mathrm{~s}$, $1 \mathrm{H}), 6.93-6.90(\mathrm{~m}, 1 \mathrm{H}), 6.44(\mathrm{~s}, 1 \mathrm{H}), 2.35(\mathrm{~s}, 3 \mathrm{H}), 2.15(\mathrm{~s}, 3 \mathrm{H}), 2.05(\mathrm{~s}, 3 \mathrm{H}) .{ }^{13} \mathrm{C}\left\{{ }^{1} \mathrm{H}\right\} \mathrm{NMR}(125$ $\mathrm{MHz}, \mathrm{CDCl}_{3}$ ): $\delta$ (ppm) 147.1, 145.0, 143.5, 137.6, 136.9, 136.5, 135.0, 133.4, 130.5, 128.9, 128.8, 128.4, 128.2, 128.1, 127.8, 126.9 (q, $J=280.6 \mathrm{~Hz}), 126.7,125.4,121.4,64.8$ (q, $J=26.6 \mathrm{~Hz})$, 21.3, 20.3, 20.0. ${ }^{19} \mathrm{~F}$ NMR (282 MHz, $\mathrm{CDCl}_{3}, \mathrm{CF}_{3} \mathrm{COOH}$-ext. st.): $\delta$ (ppm) -68.2 (s, 3F). HRMS (APPI ${ }^{+}$-Orbitrap) $m / z$ : $[\mathrm{M}]^{+}$. Calcd for $\mathrm{C}_{25} \mathrm{H}_{21} \mathrm{~F}_{3} 378.1590$; Found 378.1593 (0.8 ppm).

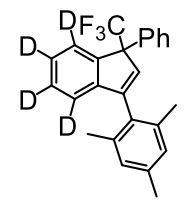

4,5,6,7-Tetradeutero-3-mesityl-1-phenyl-1-(trifluoro-methyl)-1H-indene (2a- $\left.d_{4}\right)$ was prepared according to general procedure $\mathrm{C}$ from 1,1,1-trifluoro-2-phenyl-4pentadeuterophenyl-but-3-yn-2-ol $(68.4 \mathrm{mg}, 0.243 \mathrm{mmol})$ and mesitylene $(102 \mu \mathrm{L}$, $0.73 \mathrm{mmol}, 3.0$ equiv) with $3.9 \mathrm{mg}(0.024 \mathrm{mmol})$ of $\mathrm{FeCl}_{3}$ in $0.97 \mathrm{~mL}$ of HFIP. The reaction mixture was heated at $80{ }^{\circ} \mathrm{C}$ for $24 \mathrm{~h}$. Purification by flash column chromatography over silica (petroleum ether) gave $37 \mathrm{mg}$ (39\% yield) of a colorless oil. $\mathrm{R}_{\mathrm{f}}=0.80$ (petroleum ether/EtOAc 9:1). ${ }^{1} \mathrm{H}$ NMR (400 MHz, $\left.\mathrm{CDCl}_{3}\right): \delta(\mathrm{ppm}) 7.62-7.56(\mathrm{~m}, 2 \mathrm{H}), 7.41-7.29(\mathrm{~m}, 3 \mathrm{H})$, $6.99(\mathrm{~s}, 1 \mathrm{H}), 6.95(\mathrm{~s}, 1 \mathrm{H}), 6.45(\mathrm{~s}, 1 \mathrm{H}), 2.36(\mathrm{~s}, 3 \mathrm{H}), 2.17(\mathrm{~s}, 3 \mathrm{H}), 2.07(\mathrm{~s}, 3 \mathrm{H}) .{ }^{2} \mathrm{H}$ NMR $(600$ $\mathrm{MHz}, \mathrm{CH}_{2} \mathrm{Cl}_{2}$ ): $\delta$ (ppm) 7.71 (br. s, 1D), 7.38 (br. s, 2D), 6.94 (br. s, 1D). HRMS (ESI-TOF) $m / z$ : $[\mathrm{M}+\mathrm{H}]^{+}$Calcd for $\mathrm{C}_{25} \mathrm{H}_{18} \mathrm{D}_{4} \mathrm{~F}_{3} 383.1919$; Found 383.1918 (0.3 ppm). 


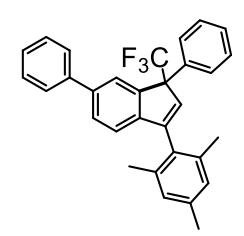

3-Mesityl-1,6-diphenyl-1-(trifluoromethyl)-1H-indene (2c) was prepared according to modified general procedure $C$ from 4-((1,1'-biphenyl)-4-yl)-1,1,1trifluoro-2-phenylbut-3-yn-2-ol (70 mg, $0.20 \mathrm{mmol})$ and mesitylene $(84 \mu \mathrm{L}, 0.60$ mmol, 3.0 equiv) with $3.3 \mathrm{mg}(0.020 \mathrm{mmol})$ of $\mathrm{FeCl}_{3}$, in $0.41 \mathrm{~mL}$ of HFIP. The reaction mixture was heated at $50{ }^{\circ} \mathrm{C}$ for $6 \mathrm{~h}$. Purification by flash column chromatography over silica (petroleum ether) gave $82 \mathrm{mg}$ (91\% yield) of the product (in $90 \%$ purity). ${ }^{1} \mathrm{H}$ NMR (400 MHz, $\left.\mathrm{CDCl}_{3}\right): \delta(\mathrm{ppm}) 7.84(\mathrm{~s}, 1 \mathrm{H}), 7.68-7.57(\mathrm{~m}, 4 \mathrm{H}), 7.54$ (dd, $J=7.9$, 1.6 Hz, 1H), 7.47-7.40 (m, 2H), 7.38-7.30 (m, 4H), 7.03-6.93 (m, 3H), $6.46(\mathrm{~s}, 1 \mathrm{H}), 2.35(\mathrm{~s}, 3 \mathrm{H})$, $2.18(\mathrm{~s}, 3 \mathrm{H}), 2.09$ (s, 3H). ${ }^{13} \mathrm{C}\left\{{ }^{1} \mathrm{H}\right\}$ NMR $\left(100 \mathrm{MHz}, \mathrm{CDCl}_{3}\right): \delta(\mathrm{ppm})$ 146.9, 144.4, 144.2, 144.1, $140.1,137.7,136.9,136.5,135.0,133.7,130.5,129.0,128.9,128.5,128.3,128.2,128.0,127.8$, $127.5,127.4,126.9$ (q, $J=280.8 \mathrm{~Hz}), 124.4,121.6,65.4(\mathrm{q}, J=26.6 \mathrm{~Hz}), 21.3,20.4,20.1 .{ }^{19} \mathrm{~F}$ NMR (376.5 MHz, CDCl $)$ ): $\delta(\mathrm{ppm})-65.9$ (s, 3F). HRMS (APPI ${ }^{+}$-Orbitrap) $m / z[\mathrm{M}]^{+}$. Calcd for $\mathrm{C}_{31} \mathrm{H}_{25} \mathrm{~F}_{3}$ 454.1903; Found 454.1904 (0.2 ppm).

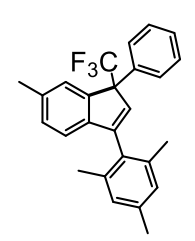

3-Mesityl-6-methyl-1-phenyl-1-(trifluoromethyl)-1H-indene (2d) was prepared according to modified general procedure $\mathrm{C}$ from 1,1,1-trifluoro-2-phenyl-4- $(p$ toluyl)but-3-yn-2-ol $(80 \mathrm{mg}, 0.28 \mathrm{mmol})$ and mesitylene $(117 \mu \mathrm{L}, 0.84 \mathrm{mmol}, 3.0$ equiv) with $4.5 \mathrm{mg}(0.028 \mathrm{mmol})$ of $\mathrm{FeCl}_{3}$, in $0.55 \mathrm{~mL}$ of HFIP. The reaction mixture was heated at $50{ }^{\circ} \mathrm{C}$ for $6 \mathrm{~h}$. Purification by flash column chromatography over silica (petroleum ether) gave $98 \mathrm{mg}\left(91 \%\right.$ yield) of the product. ${ }^{1} \mathrm{H} \mathrm{NMR}\left(400 \mathrm{MHz}, \mathrm{CDCl}_{3}\right): \delta(\mathrm{ppm})$ $7.65(\mathrm{~d}, J=7.3 \mathrm{~Hz}, 2 \mathrm{H}), 7.52(\mathrm{~s}, 1 \mathrm{H}), 7.44-7.33(\mathrm{~m}, 3 \mathrm{H}), 7.19(\mathrm{~d}, J=7.7 \mathrm{~Hz}, 1 \mathrm{H}), 7.04(\mathrm{~s}, 1 \mathrm{H})$, $7.00(\mathrm{~s}, 1 \mathrm{H}), 6.88(\mathrm{~d}, J=7.6 \mathrm{~Hz}, 1 \mathrm{H}), 6.43(\mathrm{~s}, 1 \mathrm{H}), 2.47(\mathrm{~s}, 3 \mathrm{H}), 2.40(\mathrm{~s}, 3 \mathrm{H}), 2.23(\mathrm{~s}, 3 \mathrm{H}), 2.13$ (s, 3H). ${ }^{13} \mathrm{C}\left\{{ }^{1} \mathrm{H}\right\}$ NMR $\left(100 \mathrm{MHz}, \mathrm{CDCl}_{3}\right): \delta(\mathrm{ppm})$ 147.0, 143.9, 142.4, 137.5, 136.9, 136.7, $136.5,135.3,132.5,130.7,129.6,128.8,128.4,128.2,128.0,127.8,127.0$ (q, $J=280.9 \mathrm{~Hz}), 126.3$, 121.1, 64.7 (q, $J=26.3 \mathrm{~Hz}), 21.8,21.2,20.3,20.0 .{ }^{19} \mathrm{~F} \mathrm{NMR}\left(376.5 \mathrm{MHz}, \mathrm{CDCl}_{3}\right): \delta(\mathrm{ppm})-66.0$ (s, 3F). HRMS (ESI-TOF) m/z: [M+H] ${ }^{+}$Calcd for $\mathrm{C}_{26} \mathrm{H}_{24} \mathrm{~F}_{3} 393.1825$; Found 393.1815 (2.5 ppm).

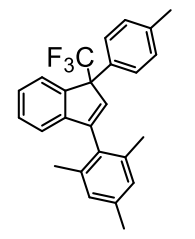

3-Mesityl-1-toluyl-1-(trifluoromethyl)-1H-indene (2f) was prepared according to general procedure C from 1,1,1-trifluoro-4-phenyl-2-( $p$-tolulyl)but-3-yn-2-ol (93 mg, $0.32 \mathrm{mmol})$ and mesitylene $(133 \mu \mathrm{L}, 0.96 \mathrm{mmol}, 3.0$ equiv $)$ with $5.1 \mathrm{mg}(0.032 \mathrm{mmol})$ of $\mathrm{FeCl}_{3}$, in $1.27 \mathrm{~mL}$ of HFIP. The reaction mixture was stirred at ambient temperature for $3 \mathrm{~h}$. Purification by flash column chromatography over silica (petroleum ether) gave $120 \mathrm{mg}$ (96\% yield) of colorless oil. $\mathrm{R}_{\mathrm{f}}=0.83$ (petroleum ether/EtOAc 9:1). ${ }^{1} \mathrm{H}$ NMR $(500$ $\left.\mathrm{MHz}, \mathrm{CDCl}_{3}\right) \delta(\mathrm{ppm}) 7.67-7.64(\mathrm{~m}, 1 \mathrm{H}), 7.48(\mathrm{~d}, J=8.0 \mathrm{~Hz}, 2 \mathrm{H}), 7.34-7.28(\mathrm{~m}, 2 \mathrm{H}), 7.16(\mathrm{~d}, J$ $=8.1 \mathrm{~Hz}, 2 \mathrm{H}), 6.99(\mathrm{~s}, 1 \mathrm{H}), 6.95(\mathrm{~s}, 1 \mathrm{H}), 6.93-6.90(\mathrm{~m}, 1 \mathrm{H}), 6.44(\mathrm{~s}, 1 \mathrm{H}), 2.35(\mathrm{~s}, 3 \mathrm{H}), 2.34(\mathrm{~s}$, $3 \mathrm{H}), 2.16(\mathrm{~s}, 3 \mathrm{H}), 2.06(\mathrm{~s}, 3 \mathrm{H}) .{ }^{13} \mathrm{C}\left\{{ }^{1} \mathrm{H}\right\} \mathrm{NMR}\left(125 \mathrm{MHz}, \mathrm{CDCl}_{3}\right) \delta(\mathrm{ppm}) 146.9,144.9,143.6$, $137.9,137.6,136.9,136.5,133.5,131.9,130.6,129.5,128.8,128.4,128.2,127.7,126.9(\mathrm{q}, J=$ $282.5 \mathrm{~Hz}), 126.6,125.4,121.3,64.5$ (q, $J=26.7 \mathrm{~Hz}), 21.2,21.1,20.3,20.0 .{ }^{19} \mathrm{~F} \mathrm{NMR}(376.5 \mathrm{MHz}$, $\mathrm{CDCl}_{3}, \mathrm{C}_{6} \mathrm{~F}_{6}$-ext. st.): $\delta(\mathrm{ppm})-68.4(\mathrm{~s}, 3 \mathrm{~F})$. HRMS (APPI ${ }^{+}$-Orbitrap) $m / z:[\mathrm{M}]^{+}$. Calcd for $\mathrm{C}_{26} \mathrm{H}_{23} \mathrm{~F}_{3}$ 392.1746; Found 392.1747 (0.3 ppm). 


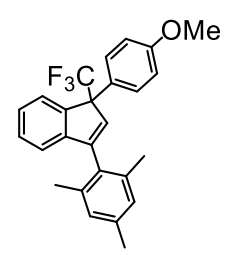

3-Mesityl-1-(4-methoxyphenyl)-1-(trifluoromethyl)-1H-indene (2j) was prepared according to modified general procedure $\mathrm{C}$ from 1,1,1-trifluoro-2-(4metoxyphenyl)-4-phenylbut-3-yn-2-ol (109 $\mathrm{mg}, 0.356 \mathrm{mmol})$ and mesitylene (149 $\mu \mathrm{L}, 1.07 \mathrm{mmol}, 3.0$ equiv) with $5.8 \mathrm{mg}(0.036 \mathrm{mmol})$ of $\mathrm{FeCl}_{3}$, in $1.42 \mathrm{~mL}$ of HFIP. The reaction mixture was heated at $80{ }^{\circ} \mathrm{C}$ for $1 \mathrm{~h}$. Purification by flash column chromatography over silica (petroleum ether/EtOAc 40:1 to 30:1) gave $131 \mathrm{mg}$ ( $90 \%$ yield) of yellow oil. $\mathrm{R}_{\mathrm{f}}=0.58$ (petroleum ether/EtOAc 9:1). ${ }^{1} \mathrm{H} \mathrm{NMR}\left(400 \mathrm{MHz}, \mathrm{CDCl}_{3}\right) \delta \mathrm{ppm} 7.69-7.63$ (m, 1H), 7.54-7.48 (d, $J=8.8 \mathrm{~Hz}, 2 \mathrm{H}), 7.31(\mathrm{dd}, J=5.5,3.1 \mathrm{~Hz}, 2 \mathrm{H}), 6.98(\mathrm{~s}, 1 \mathrm{H}), 6.94(\mathrm{~s}, 1 \mathrm{H})$, $6.91(\mathrm{dd}, J=5.5,3.2 \mathrm{~Hz}, 1 \mathrm{H}), 6.87(\mathrm{~d}, J=8.9 \mathrm{~Hz}, 2 \mathrm{H}), 6.43(\mathrm{~s}, 1 \mathrm{H}), 3.80(\mathrm{~s}, 3 \mathrm{H}), 2.35(\mathrm{~s}, 3 \mathrm{H})$, 2.15 (s, 3H), 2.04 (s, 3H). ${ }^{13} \mathrm{C}\left\{{ }^{1} \mathrm{H}\right\}$ NMR $\left(100 \mathrm{MHz}, \mathrm{CDCl}_{3}\right) \delta(\mathrm{ppm}) 159.3,146.8,144.9,143.6$, $137.6,136.9,136.5,133.5,130.6,129.1,128.8,128.4,128.2,126.7,126.9$ (q, $J=282.5 \mathrm{~Hz}), 126.6$, $125.4,121.4,114.1,64.2(\mathrm{q}, J=26.8 \mathrm{~Hz}), 55.4,21.2,20.3,20.0 .{ }^{19} \mathrm{~F}$ NMR $\left(376.5 \mathrm{MHz}, \mathrm{CDCl}_{3}\right.$, $\mathrm{CF}_{3} \mathrm{COOH}$-ext. st.): $\delta$ (ppm) -66.6 (s, 3F). HRMS (APPI ${ }^{+}$-Orbitrap): $m / z:[\mathrm{M}]^{+}$. Calcd for $\mathrm{C}_{26} \mathrm{H}_{23} \mathrm{~F}_{3} \mathrm{O}$ 408.1696; Found 408.1697 (0.2 ppm).

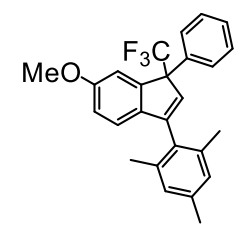

3-Mesityl-5-methoxy-1-phenyl-1-(trifluoromethyl)-1H-indene (2k) was prepared according to general procedure $\mathrm{C}$ from 1,1,1-trifluoro-4-metoxyphenyl-2phenylbut-3-yn-2-ol (106 mg, $0.345 \mathrm{mmol})$ and mesitylene $(144 \mu \mathrm{L}, 1.03 \mathrm{mmol}$, 3.0 equiv) with $5.6 \mathrm{mg}(0.035 \mathrm{mmol})$ of $\mathrm{FeCl}_{3}$, in $1.03 \mathrm{~mL}$ of HFIP. The reaction mixture was heated at $80{ }^{\circ} \mathrm{C}$ for $24 \mathrm{~h}$. Purification by flash column chromatography over silica (petroleum ether/EtOAc 40:1 to $30: 1)$ gave $84 \mathrm{mg}$ (60\% yield) of acolorless oil. $\mathrm{R}_{\mathrm{f}}=$ 0.74 (petroleum ether/EtOAc 9:1). ${ }^{1} \mathrm{H}$ NMR $\left(400 \mathrm{MHz}, \mathrm{CDCl}_{3}\right) \delta 7.56(\mathrm{~d}, J=7.8 \mathrm{~Hz}, 2 \mathrm{H}), 7.35$ $7.25(\mathrm{~m}, 3 \mathrm{H}), 7.19(\mathrm{~s}, 1 \mathrm{H}), 6.95(\mathrm{~s}, 1 \mathrm{H}), 6.91(\mathrm{~s}, 1 \mathrm{H}), 6.80(\mathrm{~s}, 2 \mathrm{H}), 6.28(\mathrm{~s}, 1 \mathrm{H}), 3.78(\mathrm{~s}, 3 \mathrm{H}), 2.31$ (s, 3H), 2.14 (s, 3H), 2.06 (s, 3H). ${ }^{13} \mathrm{C}\left\{{ }^{1} \mathrm{H}\right\}$ NMR (100 MHz, $\left.\mathrm{CDCl}_{3}\right): \delta(\mathrm{ppm})$ 159.1, 146.7, 145.4, $137.8,137.5,136.9,136.5,135.2,131.4,130.8,128.8,128.4,128.2,128.1,127.7,126.9$ (q, $J=$ $282.5 \mathrm{~Hz}), 121.8,113.5,112.7,64.7$ (q, $J=26.7 \mathrm{~Hz}), 55.7,21.2,20.3,20.0 .{ }^{19} \mathrm{~F}$ NMR $(376.5 \mathrm{MHz}$, $\mathrm{CDCl}_{3}, \mathrm{CF}_{3} \mathrm{COOH}$-ext. st.): $\delta$ (ppm) -74.9 (s, 3F). HRMS (APPI+-Orbitrap) $m / z$ : $[\mathrm{M}]^{+}$. Calcd for $\mathrm{C}_{26} \mathrm{H}_{23} \mathrm{~F}_{3} \mathrm{O}$ 408.1696; Found 408.1695 (-0.2 ppm).

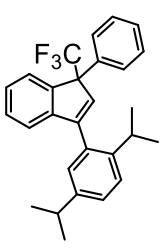

3-(2,5-Diisopropylphenyl)-1-phenyl-1-(trifluoromethyl)-1H-indene (21) was prepared according to general procedure $\mathrm{C}$ from 1,1,1-trifluoro-2,4-diphenylbut-3-yn-2-ol (104 $\mathrm{mg}, 0.375 \mathrm{mmol})$ and diizopropyl benzene $(213 \mu \mathrm{L}, 1.13 \mathrm{mmol}, 3.00$ equiv) with 6.1 $\mathrm{mg}(0.038 \mathrm{mmol})$ of $\mathrm{FeCl}_{3}$, in $0.75 \mathrm{~mL}$ of HFIP. The reaction mixture was stirred at ambient temperature for $3 \mathrm{~h}$. Purification by flash column chromatography over silica (petroleum ether) gave $142 \mathrm{mg}$ ( $90 \%$ yield) of a white solid. $\mathrm{R}_{\mathrm{f}}=0.91$ (petroleum ether/EtOAc 9:1). ${ }^{1} \mathrm{H}$ NMR $\left(500 \mathrm{MHz}, \mathrm{CDCl}_{3}\right) \delta(\mathrm{ppm}) 7.37-7.20(\mathrm{~m}, 11 \mathrm{H}), 7.17(\mathrm{~d}, J=8.3 \mathrm{~Hz}, 1 \mathrm{H}), 6.15(\mathrm{~s}$, $1 \mathrm{H}), 2.94-2.85(\mathrm{~m}, 2 \mathrm{H}), 1.15(\mathrm{~d}, J=6.8 \mathrm{~Hz}, 3 \mathrm{H}), 1.00(\mathrm{~d}, J=7.4 \mathrm{~Hz}, 3 \mathrm{H}), 0.98$ (d, $J=7.4 \mathrm{~Hz}$, $3 \mathrm{H}), 0.66(\mathrm{~d}, J=6.7 \mathrm{~Hz}, 3 \mathrm{H}) \cdot{ }^{13} \mathrm{C}\left\{{ }^{1} \mathrm{H}\right\} \mathrm{NMR}\left(126 \mathrm{MHz}, \mathrm{CDCl}_{3}\right) \delta(\mathrm{ppm}) 148.5,143.7,141.8$, $141.1,141.0,138.1,136.4$ (q, $J=2.1 \mathrm{~Hz}), 134.8,131.9,128.7$ (q, $J=2.0 \mathrm{~Hz}), 128.2,128.1,127.9$, 127.8, 126.7, 126.8 (q, $J=283.5 \mathrm{~Hz}), 126.1,125.7,121.5,64.4$ (q, $J=26.4 \mathrm{~Hz}), 29.3,27.1,24.3$, 23.9, 23.6, 23.4. ${ }^{19} \mathrm{~F}$ NMR (376.5 MHz, $\mathrm{CDCl}_{3}, \mathrm{CF}_{3} \mathrm{COOH}$-ext. st.): $\delta$ (ppm) -63.7 (s, 3F). HRMS (APPI ${ }^{+}$-Orbitrap) $\mathrm{m} / z$ : [M] ${ }^{+}$. Calcd for $\mathrm{C}_{28} \mathrm{H}_{27} \mathrm{~F}_{3}$ 420.2059; Found 420.2061 (1.9 ppm). 


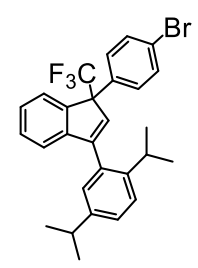

1-(4-Bromophenyl)-3-(2,5-diisopropylphenyl)-1-(trifluoromethyl)-1H-indene (2n) was prepared according to modified general procedure $C$ from 4-(4-bromophenyl)1,1,1-trifluoro-2,4-diphenylbut-3-yn-2-ol (102 $\mathrm{mg}, \quad 0.288 \quad \mathrm{mmol}) \quad$ and diisopropylbenzene $(164 \mu \mathrm{L}, 0.87 \mathrm{mmol}, 3.0$ equiv) with $4.7 \mathrm{mg}(0.029 \mathrm{mmol})$ of $\mathrm{FeCl}_{3}$, in $1.15 \mathrm{~mL}$ of HFIP. The reaction mixture was heated at $80{ }^{\circ} \mathrm{C}$ for $1 \mathrm{~h}$. Purification by flash column chromatography over silica (with petroleum ether) gave $111 \mathrm{mg}\left(77 \%\right.$ yield) of yellow solid. $\mathrm{R}_{\mathrm{f}}=0.88$ (petroleum ether/EtOAc 9:1). ${ }^{1} \mathrm{H}$ NMR $(500 \mathrm{MHz}$, $\left.\mathrm{CDCl}_{3}\right): \delta(\mathrm{ppm}) 7.57-7.29(\mathrm{~m}, 8 \mathrm{H}), 7.22-7.12(\mathrm{~m}, 3 \mathrm{H}), 6.11(\mathrm{~s}, 1 \mathrm{H}), 2.88(\mathrm{sept}, J=6.4 \mathrm{~Hz}, 2 \mathrm{H})$, $1.17(\mathrm{~d}, J=6.8 \mathrm{~Hz}, 3 \mathrm{H}), 1.02(\mathrm{~d}, J=6.8 \mathrm{~Hz}, 3 \mathrm{H}), 0.99$ (d, $J=6.8 \mathrm{~Hz}, 3 \mathrm{H}), 0.74$ (d, $J=6.8 \mathrm{~Hz}$, $3 \mathrm{H}) .{ }^{13} \mathrm{C}\left\{{ }^{1} \mathrm{H}\right\} \mathrm{NMR}\left(126 \mathrm{MHz}, \mathrm{CDCl}_{3}\right) \delta(\mathrm{ppm}) 148.5,143.7,141.8,141.1,141.0,138.1,136.4$ $(\mathrm{q}, J=1.7 \mathrm{~Hz}), 134.8,131.9,128.7(\mathrm{q}, J=1.7 \mathrm{~Hz}), 128.2,128.1,127.9,127.8,126.8(\mathrm{q}, J=281.8$ $\mathrm{Hz}), 126.7,126.1,125.7,121.5,64.4$ (q, $J=26.4 \mathrm{~Hz}), 29.4,27.1,24.3,23.9,23.6,23.4 \mathrm{z} .{ }^{19} \mathrm{~F}$ NMR (376.5 MHz, $\mathrm{CDCl}_{3}, \mathrm{CF}_{3} \mathrm{COOH}$-ext. st.): $\delta$ (ppm) -64.5 (s, 3F). HRMS (APPI $\left.{ }^{+}-O r b i t r a p\right): ~ m / z$ for $\mathrm{C}_{28} \mathrm{H}_{26}{ }^{81} \mathrm{BrF}_{3}[\mathrm{M}]^{+}$: calculated 500.1150; found 500.1145 (-1.0 ppm).

3-(4-(1-Chloro-3,5-dimethylphenyl))-1-phenyl-1-(trifluoro-methyl)-1H-indene was prepared according to a modification of general procedure B from 1,1,1-trifluoro2,4-diphenylbut-3-yn-2-ol (113 mg, $0.410 \mathrm{mmol})$ and 5-chloro- $m$-xylene (165 $\mu \mathrm{L}, 1.23$ mmol, 3.0 equiv) with $6.1 \mathrm{mg}(0.041 \mathrm{mmol})$ of $\mathrm{FeCl}_{3}$, in $1.6 \mathrm{~mL}$ of HFIP. The reaction mixture was heated at $80^{\circ} \mathrm{C}$ for $24 \mathrm{~h}$. Purification by flash column chromatography over silica (petroleum ether) gave $31 \mathrm{mg}$ (19\% yield) of a white solid. $\mathrm{R}_{\mathrm{f}}=0.31$ (petroleum ether). ${ }^{1} \mathrm{H}$ NMR (500 MHz, $\left.\mathrm{CDCl}_{3}\right): \delta(\mathrm{ppm}): 7.64(\mathrm{~d}, J=6.8 \mathrm{~Hz}, 1 \mathrm{H}), 7.55(\mathrm{~d}, J=6.8 \mathrm{~Hz}, 2 \mathrm{H}), 7.37-7.27$ $(\mathrm{m}, 5 \mathrm{H}), 7.16(\mathrm{~s}, 1 \mathrm{H}), 6.98(\mathrm{~s}, 1 \mathrm{H}), 6.95-6.91(\mathrm{~m}, 1 \mathrm{H}), 6.51(\mathrm{~s}, 1 \mathrm{H}), 2.33(\mathrm{~s}, 3 \mathrm{H}), 2.05(\mathrm{~s}, 3 \mathrm{H})$. ${ }^{13} \mathrm{C}\left\{{ }^{1} \mathrm{H}\right\} \mathrm{NMR}\left(125 \mathrm{MHz}, \mathrm{CDCl}_{3}\right): \delta$ (ppm) 144.7, 144.4, 143.2, 139.3, 138.6, 134.8, 134.6, 133.8, $129.7,129.3,128.9,128.8,128.2,127.8,127.7,126.7,126.7$ (q, $J=281.1 \mathrm{~Hz}), 125.5,121.4,65.0$ (q, $J=26.8 \mathrm{~Hz}), 21.1,20.6 .{ }^{19} \mathrm{~F} \mathrm{NMR}\left(282 \mathrm{MHz}, \mathrm{CDCl}_{3}, \mathrm{CF}_{3} \mathrm{COOH}\right.$-ext. st.): $\delta$ (ppm) -67.7 (s, 3F). HRMS (APPI+-Orbitrap) $m / z$ : [M] ${ }^{+}$. calculated for $\mathrm{C}_{24} \mathrm{H}_{18}{ }^{35} \mathrm{ClF}_{3}$ 398.1044; found 398.1050 (1.5 ppm).

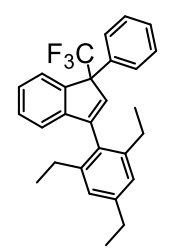

1-Phenyl-3-(2,4,6-triethylphenyl)-1-(trifluoromethyl)-1H-indene (2x) was prepared according to general procedure $\mathrm{C}$ from 1,1,1-trifluoro-2,4-diphenylbut-3-yn-2-ol (117 $\mathrm{mg}, 0.424 \mathrm{mmol})$ and 1,3,5-triethylbenzene $(239 \mu \mathrm{L}, 1.27 \mathrm{mmol}, 3.0$ equiv) with 6.9 $\mathrm{mg}(0.042 \mathrm{mmol})$ of $\mathrm{FeCl}_{3}$, in $1.69 \mathrm{~mL}$ of HFIP. The reaction mixture was heated at 80 ${ }^{\circ} \mathrm{C}$ for $24 \mathrm{~h}$. Purification by flash column chromatography over silica (petroleum ether) gave $127 \mathrm{mg}$ (71\% yield) of colorless oil. $\mathrm{R}_{\mathrm{f}}=0.88$ (petroleum ether/EtOAc 9:1). ${ }^{1} \mathrm{H}$ NMR $\left(500 \mathrm{MHz}, \mathrm{CDCl}_{3}\right) \delta(\mathrm{ppm}) 7.67-7.62(\mathrm{~m}, 1 \mathrm{H}), 7.57(\mathrm{~d}, J=6.9 \mathrm{~Hz}, 2 \mathrm{H}), 7.36-7.29(\mathrm{~m}, 5 \mathrm{H})$, $7.03(\mathrm{~s}, 1 \mathrm{H}), 7.00(\mathrm{~s}, 1 \mathrm{H}), 6.96-6.91(\mathrm{~m}, 1 \mathrm{H}), 6.48(\mathrm{~s}, 1 \mathrm{H}), 2.69(\mathrm{q}, J=7.6 \mathrm{~Hz}, 2 \mathrm{H}), 2.57-2.27(\mathrm{~m}$, 4H), $1.30(\mathrm{t}, J=7.6 \mathrm{~Hz}, 3 \mathrm{H}), 1.09$ (t, $J=7.5 \mathrm{~Hz}, 3 \mathrm{H}), 0.98(\mathrm{t}, J=7.5 \mathrm{~Hz}, 3 \mathrm{H}) .{ }^{13} \mathrm{C}\left\{{ }^{1} \mathrm{H}\right\} \mathrm{NMR}(125$ $\left.\mathrm{MHz} \mathrm{CDCl}_{3}\right) \delta(\mathrm{ppm}) 146.4,146.1,144.3,143.3,143.3,142.9,134.9,133.9,129.4,128.9,128.8$, $128.1,127.8,126.9$ (q, $J=282.5 \mathrm{~Hz}), 126.7,125.7,125.5,125.3,121.6,64.8$ (q, $J=26.7 \mathrm{~Hz})$, 28.9, 27.0, 26.9, 16.3, 16.2, 15.6. ${ }^{19} \mathrm{~F}$ NMR (282 $\mathrm{MHz}, \mathrm{CDCl}_{3}, \mathrm{CF}_{3} \mathrm{COOH}$-ext. st.): $\delta$ (ppm) -67.9 
(s, 3F). HRMS (APPI ${ }^{+}$-Orbitrap) $m / z$ : [M] ${ }^{+}$Calcd for $\mathrm{C}_{28} \mathrm{H}_{27} \mathrm{~F}_{3}$ 420.2059; Found 420.2055 (-1.2 ppm).

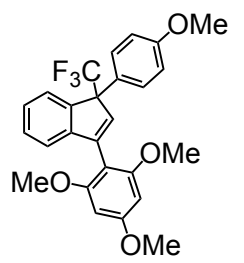

3-(2,4,6-Trimethoxyphenyl)-1-(4-methoxyphenyl)-1-(trifluoromethyl)-1H-indene (2y) was prepared according to a modification of general procedure $\mathrm{C}$ from 1,1,1trifluoro-2-(4-metoxyphenyl)-4-phenylbut-3-yn-2-ol $(258 \mathrm{mg}, 0.841 \mathrm{mmol})$ and 1,3,5-trimethoxybenzene ( $424 \mathrm{mg}, 2.52 \mathrm{mmol}, 3.0$ equiv) with $13.6 \mathrm{mg}(0.084$ $\mathrm{mmol}$ ) of $\mathrm{FeCl}_{3}$, in $3.4 \mathrm{~mL}$ of HFIP. The reaction mixture was heated at $120{ }^{\circ} \mathrm{C}$ for $24 \mathrm{~h}$ in a high-pressure reaction tube. Purification by flash column chromatography over silica (petroleum ether/EtOAc 40:1 to 30:1) gave $364 \mathrm{mg}$ (95\% yield) of a yellow solid. $\mathrm{R}_{\mathrm{f}}=0.21$ (petroleum ether/EtOAc 9:1). ${ }^{1} \mathrm{H}$ NMR $\left(500 \mathrm{MHz}, \mathrm{CDCl}_{3}\right) \delta(\mathrm{ppm}) 7.64-7.58(\mathrm{~m}, 3 \mathrm{H}), 7.30(\mathrm{td}, J$ $=1.0,7.5 \mathrm{~Hz}, 1 \mathrm{H}), 7.26-7.22(\mathrm{~m}, 1 \mathrm{H}), 7.04(\mathrm{~d}, J=7.5 \mathrm{~Hz}, 1 \mathrm{H}), 6.91-6.84(\mathrm{~m}, 2 \mathrm{H}), 6.58(\mathrm{~s}$, $1 \mathrm{H}), 6.26(\mathrm{~s}, 2 \mathrm{H}), 3.89(\mathrm{~s}, 3 \mathrm{H}), 3.79(\mathrm{~s}, 3 \mathrm{H}), 3.76-3.70(\mathrm{~m}, 6 \mathrm{H}) .{ }^{13} \mathrm{C}\left\{{ }^{1} \mathrm{H}\right\} \mathrm{NMR}\left(126 \mathrm{MHz}, \mathrm{CDCl}_{3}\right)$ $\delta(\mathrm{ppm}) 161.5,159.4,159.3,159.1,145.4,143.5,139.8,135.3$ (q, $J=1.8 \mathrm{~Hz}), 129.1$ (q, $J=1.8$ $\mathrm{Hz}), 128.2,127.4,127.0$ (q, $J=282.4 \mathrm{~Hz}), 125.8,124.7,121.9,113.9,104.8,91.2,91.1,63.9$ (q, $J=26.8 \mathrm{~Hz}), 56.2,55.9,55.5,55.3 .{ }^{19} \mathrm{~F}$ NMR $\left(282 \mathrm{MHz}, \mathrm{CDCl}_{3}, \mathrm{CF}_{3} \mathrm{COOH}\right.$-ext. st.): $\delta$ (ppm) 66.7 (s, 3F). HRMS (ESI-Orbitrap) $\mathrm{m} / z$ : [M+H] ${ }^{+}$Calcd for $\mathrm{C}_{26} \mathrm{H}_{24} \mathrm{~F}_{3} \mathrm{O}_{4} 457.1621$; Found 457.1611 $(-2.2 \mathrm{ppm})$.

\section{Preparation of tertiary propargylic alcohols for synthesis of chromenes}

General procedure D: ${ }^{33}$ Step 1. To a $0.5 \mathrm{M}$ solution of ethynyl magnesium bromide (10 mmol) in THF was slowly added aryl trifluoromethyl ketone $(10 \mathrm{mmol})$ in THF $(20 \mathrm{~mL})$. After $3 \mathrm{~h}$ at ambient temperature the reaction mixture was quenched first with water and then with saturated $\mathrm{NH}_{4} \mathrm{Cl}(\mathrm{aq})$. The aqueous layer was extracted with $\mathrm{Et}_{2} \mathrm{O}$. The combined organic layers were dried over $\mathrm{Na}_{2} \mathrm{SO}_{4}$, filtered and concentrated under reduced pressure to afford colored oil (2-aryl-1,1,1trifluoro-3-butyn-2-ol - compound A) that was engaged in the next step without further purification. Step 2. To a solution of 2-iodophenol $(10 \mathrm{mmol})$ and imidazole $(20 \mathrm{mmol})$ in dry THF $(20 \mathrm{~mL})$ was added tert-butyldimethylsilyl chloride $(20 \mathrm{mmol})$ in one portion and the reaction mixture was stirred at ambient temperature for $1 \mathrm{~h}$. The mixture was then diluted with $\mathrm{CH}_{2} \mathrm{Cl}_{2}$ and filtered through celite. The residue was purified by flash column chromatography (petroleum ether) to provide the desired product (1-iodo-2-(tert-butyldimethylsilyloxy)benzene - compound B). Step 3. To a stirred solution of compound $\mathbf{B}(10 \mathrm{mmol})$ in $\mathrm{Et}_{3} \mathrm{~N}(20 \mathrm{~mL})$ under argon were sequentially added $\mathrm{Pd}\left(\mathrm{PPh}_{3}\right)_{2} \mathrm{Cl}_{2}(1 \mathrm{~mol} \%)$ and $\mathrm{CuI}(2 \mathrm{~mol} \%)$ at ambient temperature. Then compound $\mathbf{A}$ (1.3 equiv) was added and the mixture was stirred overnight. The reaction was quenched with saturated $\mathrm{NH}_{4} \mathrm{Cl}$ (aq), extracted with $\mathrm{Et}_{2} \mathrm{O}$, dried over $\mathrm{Na}_{2} \mathrm{SO}_{4}$, and was purified by flash column chromatography (petroleum ether/EtOAc 9:1). To the isolated product (10 mmol) in THF $(20 \mathrm{~mL})$ was added tetra- $n$-butyl ammonium fluoride (1.2 equiv) at room temperature for 30 min. The reaction was quenched by adding water and extracted with EtOAc, dried over $\mathrm{Na}_{2} \mathrm{SO}_{4}$. The crude material was purified by column chromatography (petroleum ether/EtOAc 10:1) to give the pure propargylic alcohol that was used for subsequent synthesis of chromenes. 


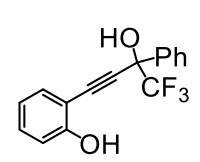

2-(4,4,4-Trifluoro-3-hydroxy-3-phenylbut-1-yn-1-yl)phenol was prepared according to general procedure $\mathrm{D}$ using 2,2,2-trifluoro-1-phenylethan-1-one (1.4 mL, $10 \mathrm{mmol})$. Purification by flash column chromatography over silica (petroleum ether/EtOAc 9:1) gave $263 \mathrm{mg}$ (90\% yield) of light yellow yellow solid. Mp: $92-93{ }^{\circ} \mathrm{C} .{ }^{1} \mathrm{H} \mathrm{NMR}$ $\left(400 \mathrm{MHz}, \mathrm{CDCl}_{3}\right): \delta(\mathrm{ppm}) 7.85-7.76(\mathrm{~m}, 2 \mathrm{H}), 7.47-7.44(\mathrm{~m}, 3 \mathrm{H}), 7.42(\mathrm{dd}, J=7.7,1.6 \mathrm{~Hz}, 1 \mathrm{H})$, $7.33(\mathrm{ddd}, J=8.2,7.5,1.5 \mathrm{~Hz}, 1 \mathrm{H}), 6.98(\mathrm{dd}, J=7.6,0.7 \mathrm{~Hz}, 1 \mathrm{H}), 6.92(\mathrm{td}, J=7.6,1.1 \mathrm{~Hz}, 1 \mathrm{H})$, $5.57(\mathrm{~s}, 1 \mathrm{H}), 3.16(\mathrm{~s}, 1 \mathrm{H}) .{ }^{13} \mathrm{C}\left\{{ }^{1} \mathrm{H}\right\} \mathrm{NMR}\left(100 \mathrm{MHz}, \mathrm{CDCl}_{3}\right): \delta(\mathrm{ppm}) 157.4,135.0,132.5,132.0$, 129.9, 128.6, 127.2, 123.4 (q, $J=285.6 \mathrm{~Hz}), 120.8,115.5,107.4,91.6,82.9,73.7$ (q, $J=32.8 \mathrm{~Hz})$. ${ }^{19} \mathrm{~F} \mathrm{NMR}$ (376.5 MHz, $\mathrm{CDCl}_{3}, \mathrm{CF}_{3} \mathrm{CO}_{2} \mathrm{H}$ - ext. st.): $\delta$ (ppm) -81.1 (s, 3F). HRMS (APPI ${ }^{+}$-Orbitrap) $m / z:[\mathrm{M}+\mathrm{H}]^{+}$Calcd for $\mathrm{C}_{16} \mathrm{H}_{12} \mathrm{O}_{2} \mathrm{~F}_{3} 293.0784$; Found 293.0783 (-0.2 ppm).

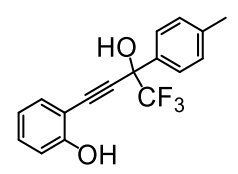

2-(4,4,4-Trifluoro-3-hydroxy-3-(p-tolyl)but-1-yn-1-yl)phenol was prepared according to general procedure D using 2,2,2-trifluoro-1-(p-tolyl)ethan-1-one (1.5 $\mathrm{mL}, 10 \mathrm{mmol})$. Purification by flash column chromatography over silica (petroleum ether/EtOAc 9:1) gave $110 \mathrm{mg}$ (36\% yield) of brown oil. ${ }^{1} \mathrm{H}$ NMR (400 $\left.\mathrm{MHz}, \mathrm{CDCl}_{3}\right): \delta(\mathrm{ppm}) 7.68(\mathrm{~d}, J=8.2 \mathrm{~Hz}, 2 \mathrm{H}), 7.41(\mathrm{dd}, J=7.7,1.4 \mathrm{~Hz}, 1 \mathrm{H}), 7.36-7.30(\mathrm{~m}, 1 \mathrm{H})$, $7.22-7.25(\mathrm{~m}, 2 \mathrm{H}), 6.97(\mathrm{~d}, J=8.3 \mathrm{~Hz}, 1 \mathrm{H}), 6.92(\mathrm{t}, J=7.6 \mathrm{~Hz}, 1 \mathrm{H}), 5.61(\mathrm{~s}, 1 \mathrm{H}), 3.19(\mathrm{~s}, 1 \mathrm{H})$, $2.40(\mathrm{~s}, 3 \mathrm{H}) .{ }^{13} \mathrm{C}\left\{{ }^{1} \mathrm{H}\right\} \mathrm{NMR}\left(100 \mathrm{MHz}, \mathrm{CDCl}_{3}\right): \delta(\mathrm{ppm}) 157.4,140.0,132.4,132.2,131.9,129.3$, $127.1,123.5$ (q, $J=285.8 \mathrm{~Hz}), 120.8,115.5,107.5,91.8,82.7,73.6$ (q, $J=31.6 \mathrm{~Hz}), 21.3 .{ }^{19} \mathrm{~F}$

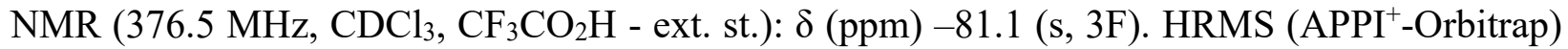
$\mathrm{m} / \mathrm{z}$ : $[\mathrm{M}]^{+}$. Calcd for $\mathrm{C}_{17} \mathrm{H}_{13} \mathrm{O}_{2} \mathrm{~F}_{3} 306.0862$; Found 305.0862 (-0.1 ppm).

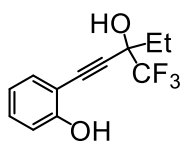

2-(3-Hydroxy-3-(trifluoromethyl)pent-1-yn-1-yl)phenol was prepared according to general procedure D using 1,1,1-trifluorobutan-2-one (1 g, $8 \mathrm{mmol})$. Purification by flash column chromatography over silica (petroleum ether/EtOAc 9:1) gave $54 \mathrm{mg}$ (22\% yield, $70 \%$ purity (remainder is 1,1,1-trifluorobutan-2-one) of brown oil. ${ }^{1} \mathrm{H}$ NMR (400 $\left.\mathrm{MHz}, \mathrm{CDCl}_{3}\right): \delta(\mathrm{ppm}) 7.36(\mathrm{dd}, J=7.7,1.6 \mathrm{~Hz}, 1 \mathrm{H}), 7.33(\mathrm{ddd}, J=8.2,7.5,1.5 \mathrm{~Hz}, 1 \mathrm{H}), 6.96(\mathrm{~d}$, $J=8.3 \mathrm{~Hz}, 2 \mathrm{H}), 6.90(\mathrm{td}, J=7.6,1.0 \mathrm{~Hz}, 1 \mathrm{H}), 5.57$ (s, 1H), $2.70(\mathrm{~s}, 1 \mathrm{H}), 1.98(\mathrm{q}, J=7.6 \mathrm{~Hz}, 2 \mathrm{H})$, $1.22(\mathrm{t}, J=7.4 \mathrm{~Hz}, 3 \mathrm{H}) .{ }^{13} \mathrm{C}\left\{{ }^{1} \mathrm{H}\right\} \mathrm{NMR}\left(100 \mathrm{MHz}, \mathrm{CDCl}_{3}\right): \delta(\mathrm{ppm}) 157.0,132.4,131.7,124.3(\mathrm{q}$, $J=285.2 \mathrm{~Hz}), 120.7,115.4,107.6,90.4,82.2,73.2$ (q, $J=31.1 \mathrm{~Hz}), 28.4,7.9 .{ }^{19} \mathrm{~F}$ NMR $(376.5$ $\mathrm{MHz}, \mathrm{CDCl}_{3}, \mathrm{CF}_{3} \mathrm{CO}_{2} \mathrm{H}$ - ext. st.): $\delta$ (ppm) -82.1 (s, 3F). HRMS (APPI+-Orbitrap) $[\mathrm{M}]^{+}$. Calcd $m / z$ : for $\mathrm{C}_{12} \mathrm{H}_{11} \mathrm{O}_{2} \mathrm{~F}_{3} 244.0711$; Found 244.0705 (-2.5 ppm).

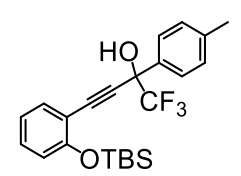

4-(2-((Tert-butyldimethylsilyl)oxy)phenyl)-1,1,1-trifluoro-2-(p-tolyl)but-3-yn-2-ol was prepared according to general procedure D using 2,2,2-trifluoro-1-(ptolyl)ethan-1-one $(1.5 \mathrm{~mL}, 10 \mathrm{mmol})$, without deprotection step. Purification by flash column chromatography over silica (petroleum ether/EtOAc 9:1) gave 362 mg (86\% yield) of brown oil. ${ }^{1} \mathrm{H}$ NMR (400 MHz, $\left.\mathrm{CDCl}_{3}\right): \delta(\mathrm{ppm}) 7.69(\mathrm{~d}, J=8.1 \mathrm{~Hz}, 2 \mathrm{H}), 7.45$ $(\mathrm{dd}, J=7.7,1.7 \mathrm{~Hz}, 1 \mathrm{H}), 7.28-7.19(\mathrm{~m}, 3 \mathrm{H}), 6.93(\mathrm{t}, J=7.5 \mathrm{~Hz}, 1 \mathrm{H}), 6.84(\mathrm{~d}, J=8.3 \mathrm{~Hz}, 1 \mathrm{H})$, $3.02(\mathrm{~s}, 1 \mathrm{H}), 2.38(\mathrm{~s}, 3 \mathrm{H}), 0.97(\mathrm{~s}, 9 \mathrm{H}), 0.24(\mathrm{~s}, 3 \mathrm{H}), 0.23(\mathrm{~s}, 3 \mathrm{H}) .{ }^{13} \mathrm{C}\left\{{ }^{1} \mathrm{H}\right\} \mathrm{NMR}(100 \mathrm{MHz}$, $\left.\mathrm{CDCl}_{3}\right): \delta(\mathrm{ppm}) 156.9,139.3,134.3,132.5,130.7,128.9,127.2,123.1$ (q, $\left.J=285.3 \mathrm{~Hz}\right), 121.1$, 119.2, 113.4, 87.8, 85.5, 73.3 (q, $J=31.9 \mathrm{~Hz}), 25.6,21.3,18.3,-4.2 .{ }^{19} \mathrm{~F}$ NMR $(376.5 \mathrm{MHz}$, 
$\mathrm{CDCl}_{3}, \mathrm{CF}_{3} \mathrm{CO}_{2} \mathrm{H}$ - ext. st.): $\delta$ (ppm) -78.9 (s, 3F). HRMS (APPI ${ }^{+}$-Orbitrap) $m / z$ : $[\mathrm{M}+\mathrm{H}]^{+}$. calculated for $\mathrm{C}_{23} \mathrm{H}_{28} \mathrm{O}_{2} \mathrm{~F}_{3} \mathrm{Si} 421.1805$; Found 421.1797 (-2.0 ppm).

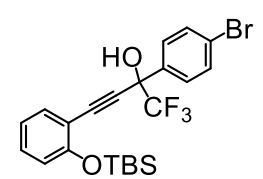

2-(4-Bromophenyl)-4-(2-((tert-butyldimethylsilyl)oxy)phenyl)-1,1,1-trifluorobut-3-yn-2-ol was prepared according to general procedure D using 1-(4bromophenyl)-2,2,2-trifluoroethan-1-one (2.5 g, $10 \mathrm{mmol})$, without deprotection step. Purification by flash column chromatography over silica (petroleum ether/EtOAc 9:1) gave $427 \mathrm{mg}$ (88\% yield) of brown oil. ${ }^{1} \mathrm{H}$ NMR $\left(400 \mathrm{MHz}, \mathrm{CDCl}_{3}\right): \delta(\mathrm{ppm})$ $7.68(\mathrm{~d}, J=8.6 \mathrm{~Hz}, 2 \mathrm{H}), 7.54(\mathrm{~d}, J=8.6 \mathrm{~Hz}, 2 \mathrm{H}), 7.44(\mathrm{dd}, J=7.7,1.7 \mathrm{~Hz}, 1 \mathrm{H}), 7.28-7.25(\mathrm{~m}$, $1 \mathrm{H}), 6.93(\mathrm{td}, J=7.6,1.0 \mathrm{~Hz}, 1 \mathrm{H}), 6.84$ (d, $J=8.3 \mathrm{~Hz}, 1 \mathrm{H}), 3.09$ (s, 1H), 0.95 (s, 9H), 0.23 (s, $3 \mathrm{H}), 0.22(\mathrm{~s}, 3 \mathrm{H}) .{ }^{13} \mathrm{C}\left\{{ }^{1} \mathrm{H}\right\} \mathrm{NMR}\left(100 \mathrm{MHz}, \mathrm{CDCl}_{3}\right): \delta(\mathrm{ppm}) 157.0,134.9,134.6,131.7,131.2$, 129.4, 124.1, 123.4 (q, $J=286.4 \mathrm{~Hz}), 121.4,119.8,113.4,87.4,86.4,73.4$ (q, $J=32.8 \mathrm{~Hz}), 25.8$, 18.5, -3.9. ${ }^{19} \mathrm{~F}$ NMR (376.5 MHz, $\mathrm{CDCl}_{3}, \mathrm{CF}_{3} \mathrm{CO}_{2} \mathrm{H}-$ ext. st.): $\delta$ (ppm) -81.0 (s, 3F). HRMS (APPI+-Orbitrap) $m / z[\mathrm{M}]^{+}$. Calcd for $\mathrm{C}_{22} \mathrm{H}_{25} \mathrm{O}_{2}{ }^{79} \mathrm{BrF}_{3} \mathrm{Si} 485.0754$; Found 485.0756 (0.4 ppm).

General procedure $\mathbf{E}$ for synthesis of $\mathbf{C F}_{3}$-chromenes and $\mathbf{C F}_{3}$-alkenes. To a solution of propargylic alcohol $(0.25 \mathrm{mmol})$ in HFIP $(125 \mu \mathrm{L})$, aryl nucleophile was added $(0.75 \mathrm{mmol})$ and $\mathrm{TfOH}(2.2 \mu \mathrm{L}, 10 \mathrm{~mol} \%)$. The reaction was stirred at $50{ }^{\circ} \mathrm{C}$ for $16 \mathrm{~h}$. The crude reaction mixture was directly transferred for silica gel chromatography.

\section{Characterization data for chromenes.}

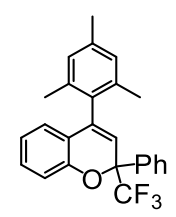

4-Mesityl-2-phenyl-2-(trifluoromethyl)-2H-chromene (4a) was prepared according to general procedure E from 2-(4,4,4-trifluoro-3-hydroxy-3-phenylbut-1-yn-1-yl)phenol $(73 \mathrm{mg}, 0.25 \mathrm{mmol})$ and mesitylene $(105 \mu \mathrm{L}, 0.750 \mathrm{mmol})$, with $2.2 \mu \mathrm{L}(0.025 \mathrm{mmol})$ of triflic acid, in $0.125 \mathrm{~mL}$ of HFIP $\left(16 \mathrm{~h}, 50{ }^{\circ} \mathrm{C}\right)$. Purification by flash column chromatography over silica (petroleum ether) gave $101 \mathrm{mg}$ (quantitative yield) of white solid. Mp: 90-92 ${ }^{\circ} \mathrm{C} .{ }^{1} \mathrm{H}$ NMR $\left(400 \mathrm{MHz}, \mathrm{CDCl}_{3}\right): \delta(\mathrm{ppm}) 7.67(\mathrm{~d}, J=7.5 \mathrm{~Hz}, 2 \mathrm{H}), 7.41-7.33$ $(\mathrm{m}, 3 \mathrm{H}), 7.20(\mathrm{t}, J=7.7 \mathrm{~Hz}, 1 \mathrm{H}), 7.11(\mathrm{~d}, J=8.1 \mathrm{~Hz}, 1 \mathrm{H}), 6.94(\mathrm{~d}, J=10.0 \mathrm{~Hz}, 2 \mathrm{H}), 6.77(\mathrm{t}, J=$ $7.5 \mathrm{~Hz}, 1 \mathrm{H}), 6.54(\mathrm{~d}, J=7.6 \mathrm{~Hz}, 1 \mathrm{H}), 6.01(\mathrm{~s}, 1 \mathrm{H}), 2.33(\mathrm{~s}, 3 \mathrm{H}), 2.13(\mathrm{~s}, 3 \mathrm{H}), 2.00(\mathrm{~s}, 3 \mathrm{H}) .{ }^{13} \mathrm{C}\left\{{ }^{1} \mathrm{H}\right\}$ NMR (100 MHz, $\left.\mathrm{CDCl}_{3}\right): \delta(\mathrm{ppm}) 151.7,137.9,137.5,136.9,133.2,130.6,129.3,128.7,128.6$, $128.5,127.1,125.7,125.0(\mathrm{q}, J=284.6 \mathrm{~Hz}), 122.5,120.9,118.3,116.9,80.4(\mathrm{q}, J=30.1 \mathrm{~Hz})$, 21.4, 20.1, 19.9. ${ }^{19} \mathrm{~F} \mathrm{NMR} \mathrm{(376.5} \mathrm{MHz,} \mathrm{CDCl}_{3} \mathrm{CF}_{3} \mathrm{CO}_{2} \mathrm{H}$, - ext. st.): $\delta$ (ppm) -80.3 (s, 3F). HRMS (APPI -Orbitrap) $m / z:[\mathrm{M}-\mathrm{H}]^{+}$for $\mathrm{C}_{25} \mathrm{H}_{20} \mathrm{OF}_{3}$ Calcd 393.1461; Found 393.1460 (-0.1 ppm).

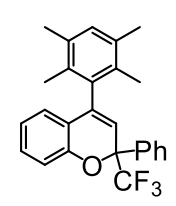

2-Phenyl-4-(2,3,5,6-tetramethylphenyl)-2-(trifluoromethyl)-2H-chromene (4b) was prepared according to general procedure E from 2-(4,4,4-trifluoro-3-hydroxy-3phenylbut-1-yn-1-yl)phenol (73 mg, $0.25 \mathrm{mmol})$ and durene (102 $\mathrm{mg}, 0.75 \mathrm{mmol})$, with $2.2 \mu \mathrm{L}(0.025 \mathrm{mmol})$ of triflic acid, in $0.125 \mathrm{~mL}$ of HFIP $\left(16 \mathrm{~h}, 50{ }^{\circ} \mathrm{C}\right)$. Purification by flash column chromatography over silica (petroleum ether) gave $88 \mathrm{mg}$ (86\% yield) of white solid. Mp: $158-160{ }^{\circ} \mathrm{C} .{ }^{1} \mathrm{H}$ NMR $\left(400 \mathrm{MHz}, \mathrm{CDCl}_{3}\right): \delta(\mathrm{ppm}) 7.66(\mathrm{~d}, J=$ $7.3 \mathrm{~Hz}, 2 \mathrm{H}), 7.42-7.33(\mathrm{~m}, 3 \mathrm{H}), 7.22-7.18(\mathrm{~m}, 1 \mathrm{H}), 7.12(\mathrm{dd}, J=8.1,0.9 \mathrm{~Hz}, 1 \mathrm{H}), 7.01$ (s, 1H), 
$6.77(\mathrm{td}, J=7.5,1.1 \mathrm{~Hz}, 1 \mathrm{H}), 6.53(\mathrm{dd}, J=7.6,1.4 \mathrm{~Hz}, 1 \mathrm{H}), 5.99$ (s, 1H), $2.26(\mathrm{~s}, 3 \mathrm{H}), 2.24$ (s, 3H), 2.05 (s, 3H), 1.89 (s, 3H). ${ }^{13} \mathrm{C}\left\{{ }^{1} \mathrm{H}\right\}$ NMR (100 MHz, $\left.\mathrm{CDCl}_{3}\right): \delta(\mathrm{ppm}) 151.6,139.0,137.5$, $136.2,134.3,134.1,132.8,132.6,131.5,130.5,129.3,128.6,127.2,126.0,124.3$ (q, $J=284.6$ $\mathrm{Hz}), 122.4,121.3,118.0,116.8,80.4(\mathrm{q}, J=30.5 \mathrm{~Hz}), 20.4,20.3,16.8,16.6 .{ }^{19} \mathrm{~F}$ NMR $(376.5$ $\mathrm{MHz}, \mathrm{CDCl}_{3}, \mathrm{CF}_{3} \mathrm{CO}_{2} \mathrm{H}$ - ext. st.): $\delta$ (ppm) -80.3 (s, 3F). HRMS (APPI ${ }^{+}$-Obitrap) $\mathrm{m} / \mathrm{z}$ : [M] ${ }^{+}$. Calcd for $\mathrm{C}_{26} \mathrm{H}_{23} \mathrm{OF}_{3}$ 408.1696; Found 408.1698 (0.6 ppm).

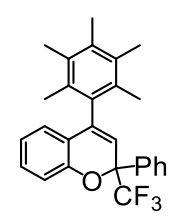

4-(2,3,4,5,6-Pentamethylphenyl)-2-phenyl-2-(trifluoromethyl)-2H-chromene (4c) was prepared according to general procedure E from 2-(4,4,4-trifluoro-3-hydroxy-3phenylbut-1-yn-1-yl)phenol (72.5 mg, $0.25 \mathrm{mmol})$ and pentamethyl-benzene $(111 \mathrm{mg}$, $0.75 \mathrm{mmol})$, with $2.2 \mu \mathrm{L}(0.025 \mathrm{mmol})$ of triflic acid, in $0.125 \mathrm{~mL}$ of HFIP (16 h, 50 $\left.{ }^{\circ} \mathrm{C}\right)$. Purification by flash column chromatography over silica (petroleum ether) gave $132 \mathrm{mg}$ (quantitative yield) of white solid. Mp: 92-94 ${ }^{\circ} \mathrm{C} .{ }^{1} \mathrm{H}$ NMR $\left(400 \mathrm{MHz}, \mathrm{CDCl}_{3}\right): \delta(\mathrm{ppm})$ $7.65(\mathrm{~d}, J=7.3 \mathrm{~Hz}, 2 \mathrm{H}), 7.44-7.30(\mathrm{~m}, 3 \mathrm{H}), 7.23-7.17(\mathrm{~m}, 1 \mathrm{H}), 7.12(\mathrm{dd}, J=8.1,1.0 \mathrm{~Hz}, 1 \mathrm{H})$, $6.77(\mathrm{td}, J=7.5,1.2 \mathrm{~Hz}, 1 \mathrm{H}), 6.56(\mathrm{dd}, J=7.6,1.5 \mathrm{~Hz}, 1 \mathrm{H}), 6.00(\mathrm{~s}, 1 \mathrm{H}), 2.30(\mathrm{~s}, 3 \mathrm{H}), 2.25$ (s, $3 \mathrm{H}), 2.23(\mathrm{~s}, 3 \mathrm{H}), 2.11(\mathrm{~s}, 3 \mathrm{H}), 1.95(\mathrm{~s}, 3 \mathrm{H}) .{ }^{13} \mathrm{C}\left\{{ }^{1} \mathrm{H}\right\} \mathrm{NMR}\left(100 \mathrm{MHz}, \mathrm{CDCl}_{3}\right): \delta(\mathrm{ppm}) 151.6$, 139.6, 137.5, 136.1 133.8, 133.2, 132.9, 132.4, 132.2, 130.5, 129.3, 128.6, 127.2, 126.2, 124.4 (q, $J=284.6 \mathrm{~Hz}), 122.4,121.6,118.1,116.8,80.5(\mathrm{q}, J=30.5 \mathrm{~Hz}), 18.1,17.8,17.2,16.9,16.8 .{ }^{19} \mathrm{~F}$ NMR (376.5 MHz, $\mathrm{CDCl}_{3}, \mathrm{CF}_{3} \mathrm{CO}_{2} \mathrm{H}$ - ext. st.): $\delta$ (ppm) -80.2 (s, 3F). HRMS (APPI+Orbitrap) $m / z$ : $[\mathrm{M}]^{+}$. Calcd for $\mathrm{C}_{27} \mathrm{H}_{25} \mathrm{OF}_{3} 422.1852$; Found 422.1855 (0.7 ppm).

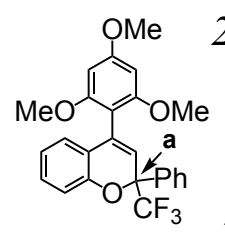

2-Phenyl-2-(trifluoromethyl)-4-(2,4,6-trimethoxyphenyl)-2H-chromene (4d) was prepared according to general procedure E from 2-(4,4,4-trifluoro-3-hydroxy-3phenylbut-1-yn-1-yl)phenol (73 mg, $0.25 \mathrm{mmol})$ and 1,3,5-trimethoxybenzene (116 $\mathrm{mg}, 0.75 \mathrm{mmol})$, with $2.2 \mu \mathrm{L}(0.025 \mathrm{mmol})$ of triflic acid, in $0.125 \mathrm{~mL}$ of HFIP (16 h, $50{ }^{\circ} \mathrm{C}$ ). Purification by flash column chromatography over silica (petroleum ether/EtOAc 97:3) gave $47 \mathrm{mg}$ (43\% yield) of white solid. Mp: $133-135{ }^{\circ} \mathrm{C} .{ }^{1} \mathrm{H} \mathrm{NMR}(400 \mathrm{MHz}$, $\left.\mathrm{CDCl}_{3}\right): \delta(\mathrm{ppm}) 7.74(\mathrm{~d}, J=7.4 \mathrm{~Hz}, 2 \mathrm{H}), 7.38-7.32(\mathrm{~m}, 3 \mathrm{H}), 7.11(\mathrm{t}, J=6.9 \mathrm{~Hz}, 1 \mathrm{H}), 7.02(\mathrm{~d}, J=$ $8.1 \mathrm{~Hz}, 1 \mathrm{H}), 6.75(\mathrm{t}, J=7.5 \mathrm{~Hz}, 1 \mathrm{H}), 6.64(\mathrm{~d}, J=7.6 \mathrm{~Hz}, 1 \mathrm{H}), 6.20(\mathrm{~d}, J=3.4 \mathrm{~Hz}, 1 \mathrm{H}), 6.20(\mathrm{~d}, J$ $=7.6 \mathrm{~Hz}, 1 \mathrm{H}), 6.13(\mathrm{~s}, 1 \mathrm{H}), 3.87(\mathrm{~s}, 3 \mathrm{H}), 3.69(\mathrm{~s}, 3 \mathrm{H}), 3.67(\mathrm{~s}, 3 \mathrm{H}) .{ }^{13} \mathrm{C}\left\{{ }^{1} \mathrm{H}\right\} \mathrm{NMR}(100 \mathrm{MHz}$, $\left.\mathrm{CDCl}_{3}\right): \delta(\mathrm{ppm}) 161.8,159.6,159.4,151.4,137.6,131.7,129.6,129.2,128.2,127.9,125.8,124.7$ $(\mathrm{q}, J=283.8 \mathrm{~Hz}), 122.4,122.0,120.5,116.8,107.3,91.3,91.2,56.3,56.1,55.7$ (quaternary carbon a displayed a very weak signal). ${ }^{19} \mathrm{~F}$ NMR $\left(376.5 \mathrm{MHz}, \mathrm{CDCl}_{3}, \mathrm{CF}_{3} \mathrm{CO}_{2} \mathrm{H}\right.$ - ext. st.): $\delta$ (ppm) 79.9 (s, 3F). HRMS (APPI ${ }^{+}$-Orbitrap) $m / z$ : $[\mathrm{M}+\mathrm{H}]^{+}$Calcd for $\mathrm{C}_{25} \mathrm{H}_{22} \mathrm{O}_{4} \mathrm{~F}_{3}$ 443.1465; Found $443.1464(-0.2 \mathrm{ppm})$.

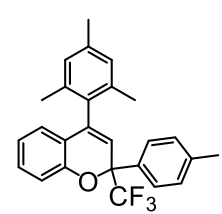

4-Mesityl-2-(p-tolyl)-2-(trifluoromethyl)-2H-chromene (4e) was prepared according to general procedure E from 2-(4,4,4-trifluoro-3-hydroxy-3-( $p$-tolyl)but1-yn-1-yl)phenol (77 mg, $0.25 \mathrm{mmol})$ and mesitylene (105 $\mu \mathrm{L}, 0.75 \mathrm{mmol})$, with $2.2 \mu \mathrm{L}(0.025 \mathrm{mmol})$ of triflic acid, in $0.125 \mathrm{~mL}$ of $\mathrm{HFIP}\left(16 \mathrm{~h}, 50^{\circ} \mathrm{C}\right)$. Purification by flash column chromatography over silica (petroleum ether) gave $55 \mathrm{mg}(54 \%$ yield) of white solid. 4e was also prepared according to general procedure E from 4-(2-((tert- 
butyldimethylsilyl)oxy)phenyl)-1,1,1-trifluoro-2-( $p$-tolyl)but-3-yn-2-ol (105 $\mathrm{mg}, 0.25 \mathrm{mmol}$ ) and mesitylene $(105 \mu \mathrm{L}, 0.75 \mathrm{mmol})$, with $2.2 \mu \mathrm{L}(0.025 \mathrm{mmol})$ of triflic acid, in $0.125 \mathrm{~mL}$ of HFIP $\left(16 \mathrm{~h}, 50^{\circ} \mathrm{C}\right)$. Purification by flash column chromatography over silica (petroleum ether) gave 290 mg (71\% yield) of white solid. Mp: $110-113{ }^{\circ} \mathrm{C} .{ }^{1} \mathrm{H}$ NMR (400 MHz, $\left.\mathrm{CDCl}_{3}\right): \delta$ (ppm) 7.55 (d, $J$ $=8.1 \mathrm{~Hz}, 2 \mathrm{H}), 7.21-7.17(\mathrm{~m}, 3 \mathrm{H}), 7.09(\mathrm{dd}, J=8.1,1.0 \mathrm{~Hz}, 1 \mathrm{H}), 6.95(\mathrm{~s}, 1 \mathrm{H}), 6.93(\mathrm{~s}, 1 \mathrm{H}), 6.76$ (td, $J=7.5,1.2 \mathrm{~Hz}, 1 \mathrm{H}), 6.54$ (dd, $J=7.6,1.5 \mathrm{~Hz}, 1 \mathrm{H}), 6.00(\mathrm{~s}, 1 \mathrm{H}), 2.34(\mathrm{~s}, 3 \mathrm{H}), 2.33(\mathrm{~s}, 3 \mathrm{H})$, $2.12(\mathrm{~s}, 3 \mathrm{H}), 2.02(\mathrm{~s}, 3 \mathrm{H}) .{ }^{13} \mathrm{C}\left\{{ }^{1} \mathrm{H}\right\} \operatorname{NMR}\left(100 \mathrm{MHz}, \mathrm{CDCl}_{3}\right): \delta(\mathrm{ppm}) 151.7,139.2,137.8,137.7$, 136.9, 134.5, 133.3, 130.5, 129.3, 128.7, 128.4, 127.1, 125.6, 124.2 (q, $J=284.6 \mathrm{~Hz}), 122.4,121.0$, $118.5,116.9,80.4$ (q, $J=30.5 \mathrm{~Hz}), 24.5,21.4,20.1,19.9 .{ }^{19} \mathrm{~F} \mathrm{NMR}\left(376.5 \mathrm{MHz}, \mathrm{CDCl}_{3}, \mathrm{CF}_{3} \mathrm{CO}_{2} \mathrm{H}\right.$ - ext. st.): $\delta(\mathrm{ppm})-80.5$ (s, 3F). HRMS (APPI ${ }^{+}$-Orbitrap) $\mathrm{m} / z$ : $[\mathrm{M}]^{+}$. Calcd for $\mathrm{C}_{26} \mathrm{H}_{23} \mathrm{OF}_{3}$ 408.1698; Found 408.1696 ( -0.7 ppm).

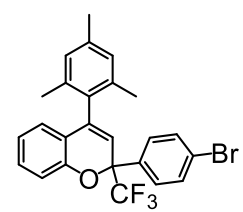

2-(4-Bromophenyl)-4-mesityl-2-(trifluoromethyl)-2H-chromene (4f) was prepared according to general procedure $\mathrm{E}$ from 2-(4-bromophenyl)-4-(2-(tertbutyldimethylsilyl)oxy)phenyl)-1,1,1-trifluoro-but-3-yn-2-ol (121 $\mathrm{mg}, \quad 0.25$ $\mathrm{mmol})$ and mesitylene $(105 \mu \mathrm{L}, 0.75 \mathrm{mmol})$, with $2.2 \mu \mathrm{L}(0.025 \mathrm{mmol})$ of triflic acid, in $0.125 \mathrm{~mL}$ of HFIP $\left(16 \mathrm{~h}, 50{ }^{\circ} \mathrm{C}\right)$. Purification by flash column chromatography over silica (petroleum ether) gave $89 \mathrm{mg}$ ( $75 \%$ yield) white solid. Mp: 113-115 ${ }^{\circ} \mathrm{C} .{ }^{1} \mathrm{H}$ NMR (400 MHz, $\left.\mathrm{CDCl}_{3}\right) \delta 7.53(\mathrm{~s}, 4 \mathrm{H}), 7.23-7.18(\mathrm{~m}, 1 \mathrm{H}), 7.09(\mathrm{~d}, J=7.9 \mathrm{~Hz}, 1 \mathrm{H}), 6.94$ $(\mathrm{d}, J=6.9 \mathrm{~Hz}, 2 \mathrm{H}), 6.79(\mathrm{t}, J=7.4 \mathrm{~Hz}, 1 \mathrm{H}), 6.55(\mathrm{dd}, J=1.2,7.7 \mathrm{~Hz}, 1 \mathrm{H}), 5.97(\mathrm{~s}, 1 \mathrm{H}), 2.33(\mathrm{~s}$, $3 \mathrm{H}), 2.11(\mathrm{~s}, 3 \mathrm{H}), 2.00(\mathrm{~s}, 3 \mathrm{H}) .{ }^{13} \mathrm{C}\left\{{ }^{1} \mathrm{H}\right\} \mathrm{NMR}\left(100 \mathrm{MHz}, \mathrm{CDCl}_{3}\right): \delta(\mathrm{ppm}) 151.4,138.4,138.0$, $136.8,136.7,136.5,133.0,131.9,130.8,128.9,128.7,128.5,125.8,124.1$ (q, $J=284.6 \mathrm{~Hz}), 123.8$, 122.7, 120.8, 117.7, 116.9, 80.1 (q, $J=30.5 \mathrm{~Hz}), 21.4,20.1,19.9 .{ }^{19} \mathrm{~F} \mathrm{NMR} \mathrm{(376.5} \mathrm{MHz,} \mathrm{CDCl}_{3}$, $\mathrm{CF}_{3} \mathrm{CO}_{2} \mathrm{H}$ - ext. st.): $\delta$ (ppm) -80.5 (s, 3F). HRMS (APPI -Orbitrap) $\mathrm{m} / z$ [M] $]^{+}$. Calcd for $\mathrm{C}_{25} \mathrm{H}_{21} \mathrm{OBrF}_{3}$ 473.0722; Found 473.0716 (-1.3 ppm).

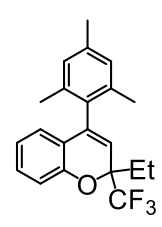

2-Ethyl-4-mesityl-2-(trifluoromethyl)-2H-chromene (4g) was prepared according to general procedure $\mathrm{E}$ from 2-(3-hydroxy-3-(trifluoromethyl)pent-1-yn-1-yl)phenol (61 $\mathrm{mg}, 0.25 \mathrm{mmol})$ and mesitylene $(105 \mu \mathrm{L}, 0.75 \mathrm{mmol})$, with $2.2 \mu \mathrm{L}(0.025 \mathrm{mmol})$ of triflic acid, in $0.125 \mathrm{~mL}$ of HFIP $\left(16 \mathrm{~h}, 50{ }^{\circ} \mathrm{C}\right)$. Purification by flash column chromatography over silica (petroleum ether) gave $67 \mathrm{mg}$ (77\% yield) of colorless oil.

${ }^{1} \mathrm{H}$ NMR $\left(400 \mathrm{MHz}, \mathrm{CDCl}_{3}\right): \delta(\mathrm{ppm}) 7.14(\mathrm{td}, J=8.0,1.6 \mathrm{~Hz}, 1 \mathrm{H}), 6.94(\mathrm{~s}, 1 \mathrm{H}), 6.92(\mathrm{~s}, 1 \mathrm{H})$, $6.90(\mathrm{dd}, J=8.1,0.8 \mathrm{~Hz}, 1 \mathrm{H}), 6.73(\mathrm{td}, J=7.5,1.1 \mathrm{~Hz}, 1 \mathrm{H}), 6.52$ (dd, $J=7.6,1.6 \mathrm{~Hz}, 1 \mathrm{H}), 5.26$ (s, 1H), $2.33(\mathrm{~s}, 3 \mathrm{H}), 2.17-2.07(\mathrm{~m}, 1 \mathrm{H}), 2.14(\mathrm{~s}, 3 \mathrm{H}), 2.09$ (s, 3H), $1.83(\mathrm{dq}, J=14.2,7.3 \mathrm{~Hz}, 1 \mathrm{H})$, $1.11(\mathrm{t}, J=7.4 \mathrm{~Hz}, 3 \mathrm{H}) .{ }^{13} \mathrm{C}\left\{{ }^{1} \mathrm{H}\right\} \mathrm{NMR}\left(100 \mathrm{MHz}, \mathrm{CDCl}_{3}\right): \delta(\mathrm{ppm}) 153.4,139.7,137.7,137.1$, $136.3,133.5,130.4,128.7,128.4,125.5,125.0$ (q, $J=284.6 \mathrm{~Hz}), 121.8,119.8,117.4,115.8,80.7$ (q, $J=30.5 \mathrm{~Hz}), 27.9,21.4,20.2,19.8,7.8 .{ }^{19} \mathrm{~F} \mathrm{NMR}\left(376.5 \mathrm{MHz}, \mathrm{CDCl}_{3}, \mathrm{CF}_{3} \mathrm{CO}_{2} \mathrm{H}\right.$ - ext. st.): $\delta$ (ppm) -83.0 (s, 3F). HRMS (APPI ${ }^{+}$-Orbitrap) $m / z$ : [M] $]^{+.}$Calcd for $\mathrm{C}_{21} \mathrm{H}_{21} \mathrm{OF}_{3}$ 346.1539; Found $346.1538(-0.3 \mathrm{ppm})$.

General procedure $F$ for preparation of secondary propargylic alcohols. Secondary propargylic alcohols were prepared via two-step Kitazume/Sato sequence. ${ }^{34}$ To a mixture of alkyne 
(10 mmol) and anhydrous THF $(30 \mathrm{~mL})$ at $-78^{\circ} \mathrm{C}$ was added $n$-BuLi (10 mmol, $2.5 \mathrm{M}$ solution) for $5 \mathrm{~min}$. After $20 \mathrm{~min}$ stirring at $-78{ }^{\circ} \mathrm{C}$, ethyl fluoroacetate $(10 \mathrm{mmol})$, boron trifluoride diethyl etherate $(12 \mathrm{mmol})$, and anhydrous THF $(20 \mathrm{~mL})$ were added. After an additional $2 \mathrm{~h}$ of stirring, the reaction was quenched with brine, extracted with ethyl acetate, and dried over $\mathrm{Na}_{2} \mathrm{SO}_{4}$. The resulting ketone was purified by flash chromatography (petroleum ether/EtOAc 9:1). The ketone was dissolved in methanol $(10 \mathrm{~mL})$. To the solution was added $\mathrm{NaBH}_{4}(10 \mathrm{mmol})$ slowly and the reaction solution was stirred for $30 \mathrm{~min}$ at room temperature. The mixture was quenched by adding brine and extracted with ethyl acetate, dried over $\mathrm{Na}_{2} \mathrm{SO}_{4}$. Finally, purification by flash chromatography yielded the secondary propargylic alcohols.

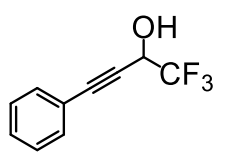

1,1,1-Trifluoro-4-phenylbut-3-yn-2-ol was prepared according to general procedure $\mathrm{F}$ using phenylacetylene $(0.54 \mathrm{~mL}, 5.0 \mathrm{mmol})$. Purification by flash column chromatography over silica (petroleum ether/EtOAc 9:1) gave $88 \mathrm{mg}(44 \%$ yield) of yellow oil. The experimental data are in agreement with the literature. ${ }^{35}$ ${ }^{1} \mathrm{H}$ NMR (400 MHz, $\left.\mathrm{CDCl}_{3}\right): \delta(\mathrm{ppm}) 7.50-7.48(\mathrm{~m}, 2 \mathrm{H}), 7.42-7.32(\mathrm{~m}, 3 \mathrm{H}), 4.94-4.88(\mathrm{~m}, 1 \mathrm{H})$, $2.52(\mathrm{~d}, J=8.3 \mathrm{~Hz}, 1 \mathrm{H}) .{ }^{13} \mathrm{C}\left\{{ }^{1} \mathrm{H}\right\}$ NMR $\left(100 \mathrm{MHz}, \mathrm{CDCl}_{3}\right): \delta(\mathrm{ppm}) 132.4,129.9,128.8,123.2$ $(\mathrm{q}, J=281.9 \mathrm{~Hz}), 121.2,88.4,80.7,63.3(\mathrm{q}, J=36.5 \mathrm{~Hz}) .{ }^{19} \mathrm{~F} \mathrm{NMR}\left(376.5 \mathrm{MHz}, \mathrm{CDCl}_{3}, \mathrm{CF}_{3} \mathrm{CO}_{2} \mathrm{H}\right.$ - ext. st.): $\delta$ (ppm) -80.2 (s, 3F).

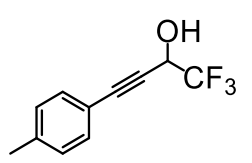

1,1,1-Trifluoro-4-(p-tolyl)but-3-yn-2-ol was prepared according to general procedure F using 1-ethynyl-4-methylbenzene $(0.63 \mathrm{~mL}, 5.0 \mathrm{mmol})$. Purification by flash column chromatography over silica (petroleum ether/EtOAc 9:1) gave 98 mg (46\% yield) of white solid. Mp: $69-71{ }^{\circ} \mathrm{C} .{ }^{1} \mathrm{H}$ NMR (400 MHz, $\left.\mathrm{CDCl}_{3}\right): \delta$ (ppm) 7.40 (d, $J=8.0 \mathrm{~Hz}, 2 \mathrm{H}), 7.17$ (d, $J=7.9 \mathrm{~Hz}, 2 \mathrm{H}), 4.95-4.89(\mathrm{~m}, 1 \mathrm{H}), 2.47$ (d, $J=6.7 \mathrm{~Hz}$, $1 \mathrm{H}), 2.39(\mathrm{~s}, 3 \mathrm{H}) .{ }^{13} \mathrm{C}\left\{{ }^{1} \mathrm{H}\right\} \operatorname{NMR}\left(100 \mathrm{MHz}, \mathrm{CDCl}_{3}\right): \delta(\mathrm{ppm}) 140.2,132.3,129.5,123.1(\mathrm{q}, J=$ $281.7 \mathrm{~Hz}), 118.1,88.6,80.1,63.3$ (q, $J=36.4 \mathrm{~Hz}), 21.6 .{ }^{19} \mathrm{~F} \mathrm{NMR}\left(376.5 \mathrm{MHz}, \mathrm{CDCl}_{3}, \mathrm{CF}_{3} \mathrm{CO}_{2} \mathrm{H}\right.$ - ext. st.): $\delta(\mathrm{ppm})-80.3$ (s, 3F). HRMS (APPI ${ }^{+}$-Orbitrap) $m / z$ : for $\mathrm{C}_{11} \mathrm{H}_{10} \mathrm{OF}_{3}[\mathrm{M}+\mathrm{H}]^{+}$. Calcd 215.0678; found 215.0678 ( $-0.0 \mathrm{ppm})$.

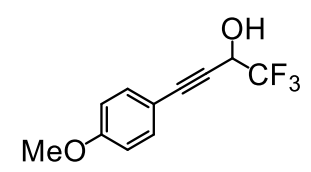

1,1,1-Trifluoro-4-(4-methoxyphenyl)but-3-yn-2-ol was prepared according to general procedure $\mathrm{F}$ using 1-ethynyl-4-methoxybenzene $(0.65 \mathrm{~mL}, 5.0 \mathrm{mmol})$. Purification by flash column chromatography over silica (petroleum ether/EtOAc 85:15) gave $69 \mathrm{mg}$ (30\% yield) of yellow oil. ${ }^{1} \mathrm{H} \mathrm{NMR}(400 \mathrm{MHz}$, $\left.\mathrm{CDCl}_{3}\right) \delta(\mathrm{ppm}) 7.44-7.39(\mathrm{~m}, 2 \mathrm{H}), 6.89-6.83(\mathrm{~m}, 2 \mathrm{H}), 4.89(\mathrm{dq}, J=5.7,8.2 \mathrm{~Hz}, 1 \mathrm{H}), 3.82(\mathrm{~s}$, $3 \mathrm{H}), 2.47(\mathrm{~d}, J=8.3 \mathrm{~Hz}, 1 \mathrm{H}) .{ }^{13} \mathrm{C}\left\{{ }^{1} \mathrm{H}\right\} \mathrm{NMR}\left(100 \mathrm{MHz}, \mathrm{CDCl}_{3}\right): \delta(\mathrm{ppm}) 160.8,133.9,123.3(\mathrm{q}$, $J=282.0 \mathrm{~Hz}), 114.4,113.2,88.4,79.5,63.3$ (q, $J=36.7 \mathrm{~Hz}), 55.7 .{ }^{19} \mathrm{~F} \mathrm{NMR}\left(376.5 \mathrm{MHz}, \mathrm{CDCl}_{3}\right.$, $\mathrm{CF}_{3} \mathrm{CO}_{2} \mathrm{H}$ - ext. st.): $\delta(\mathrm{ppm})-78.3$ (s, 3F). HRMS $\left(\mathrm{APPI}^{+}\right): \mathrm{m} / z$ for $\mathrm{C}_{11} \mathrm{H}_{9} \mathrm{O}_{2} \mathrm{~F}_{3}\left([\mathrm{M}]^{+}\right)$: calculated 230.0555; found $230.0551(-1.7 \mathrm{ppm})$.

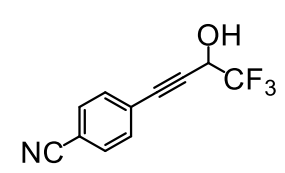

4-(4,4,4-Trifluoro-3-hydroxybut-1-yn-1-yl)benzonitrile was prepared according to general procedure $\mathrm{F}$ using 1-ethynyl-4-isocyanobenzene $(0.64 \mathrm{~g}, 5.0 \mathrm{mmol})$. Purification by flash column chromatography over silica (petroleum ether/EtOAc 9:1) gave $56 \mathrm{mg}$ (25\% yield) of yellow solid. Mp: $90-92{ }^{\circ} \mathrm{C} .{ }^{1} \mathrm{H}$ 
NMR (400 MHz, $\left.\mathrm{CDCl}_{3}\right): \delta(\mathrm{ppm}) 7.65(\mathrm{~d}, J=8.1 \mathrm{~Hz}, 2 \mathrm{H}), 7.58(\mathrm{~d}, J=8.4 \mathrm{~Hz}, 2 \mathrm{H}), 4.98-4.91$ $(\mathrm{m}, 1 \mathrm{H}), 2.59(\mathrm{~d}, J=8.4 \mathrm{~Hz}, 1 \mathrm{H}) .{ }^{13} \mathrm{C}\left\{{ }^{1} \mathrm{H}\right\} \mathrm{NMR}\left(101 \mathrm{MHz}, \mathrm{CDCl}_{3}\right) \delta 132.7,132.3,125.8,122.7$ (q, $J=282.0 \mathrm{~Hz}), 118.2,113.1,86.1,84.6,63.1$ (q, $J=36.8 \mathrm{~Hz}) .{ }^{19} \mathrm{~F} \mathrm{NMR}\left(376.5 \mathrm{MHz}, \mathrm{CDCl}_{3}\right.$, $\mathrm{CF}_{3} \mathrm{CO}_{2} \mathrm{H}$ - ext. st.): $\delta$ (ppm) -80.0 (s, 3F). HRMS (APPI ${ }^{+}$-Orbitrap) $m / z$ : $[\mathrm{M}+\mathrm{H}]^{+}$Calcd for $\mathrm{C}_{11} \mathrm{H}_{7} \mathrm{ONF}_{3}$ 226.0480; Found 226.0475 (-2.3 ppm).

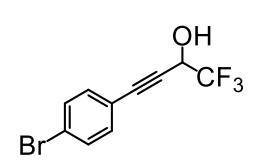

4-(4-Bromophenyl)-1,1,1-trifluorobut-3-yn-2-ol was prepared according to general procedure $\mathrm{F}$ using 1-ethynyl-4-bromobenzene $(0.60 \mathrm{~mL}, 5.0 \mathrm{mmol})$. Purification by flash column chromatography over silica (petroleum ether/EtOAc 9:1) gave $167 \mathrm{mg}$ (60\% yield) of yellow/dark yellow solid. Mp: $62-63{ }^{\circ} \mathrm{C} .{ }^{1} \mathrm{H}$ NMR (400 MHz, CDCl $): \delta(p p m) 7.49(\mathrm{~d}, J=8.4 \mathrm{~Hz}, 2 \mathrm{H}), 7.34(\mathrm{~d}, J=8.4 \mathrm{~Hz}, 2 \mathrm{H}), 4.91-4.88$ $(\mathrm{m}, 1 \mathrm{H}), 2.50(\mathrm{~d}, J=7.0 \mathrm{~Hz}, 1 \mathrm{H}) .{ }^{13} \mathrm{C}\left\{{ }^{1} \mathrm{H}\right\} \mathrm{NMR}\left(100 \mathrm{MHz}, \mathrm{CDCl}_{3}\right): \delta(\mathrm{ppm}) 133.8,132.2,124.4$, $123.1(\mathrm{q}, J=282.4 \mathrm{~Hz}), 120.1,87.2,81.8,62.3(\mathrm{q}, J=36.6 \mathrm{~Hz}) .{ }^{19} \mathrm{~F}$ NMR $\left(376.5 \mathrm{MHz}, \mathrm{CDCl}_{3}\right.$, $\mathrm{CF}_{3} \mathrm{CO}_{2} \mathrm{H}$ - ext. st.): $\delta$ (ppm) -80.2 (s, 3F). HRMS (APPI ${ }^{+}$-Orbitrap) $m / z$ : $[\mathrm{M}]^{+}$. Calcd for $\mathrm{C}_{10} \mathrm{H}_{5} \mathrm{O}^{79} \mathrm{BrF}_{3} 276.9470$; found 276.9473 (0.8 ppm).

\section{Characterization data for Friedel-Crafts reaction products of secondary propargylic alcohols}

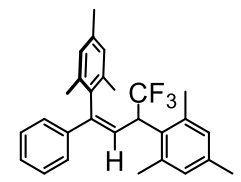

(Z)-2,2'-(4,4,4-Trifluoro-1-phenylbut-1-ene-1,3-diyl)bis(1,3,5-trimethylbenzene) (6a) was prepared according to general procedure E from 1,1,1-trifluoro-4phenylbut-3-yn-2-ol (50 mg, $0.25 \mathrm{mmol})$ and mesitylene $(105 \mu \mathrm{L}, 0.75 \mathrm{mmol})$, with $2.2 \mu \mathrm{L}(0.025 \mathrm{mmol})$ of triflic acid, in $0.125 \mathrm{~mL}$ of HFIP $\left(16 \mathrm{~h}, 50{ }^{\circ} \mathrm{C}\right)$.

Purification by flash column chromatography over silica (petroleum ether) gave $80 \mathrm{mg}$ (75\% yield) of white solid. Mp: $135-136{ }^{\circ} \mathrm{C} .{ }^{1} \mathrm{H}$ NMR $\left(400 \mathrm{MHz}, \mathrm{CDCl}_{3}\right): \delta(\mathrm{ppm}) 7.27-7.21(\mathrm{~m}, 5 \mathrm{H}), 6.95(\mathrm{~s}$, $1 \mathrm{H}), 6.92(\mathrm{~d}, J=8.7 \mathrm{~Hz}, 1 \mathrm{H}), 6.79(\mathrm{~s}, 1 \mathrm{H}), 6.76(\mathrm{~s}, 1 \mathrm{H}), 6.65(\mathrm{~d}, J=5.1 \mathrm{~Hz}, 1 \mathrm{H}), 4.38-4.29(\mathrm{~m}$, 1H), $2.51(\mathrm{~s}, 3 \mathrm{H}), 2.28(\mathrm{~s}, 3 \mathrm{H}), 2.27(\mathrm{~s}, 3 \mathrm{H}), 2.19(\mathrm{~s}, 3 \mathrm{H}), 2.18(\mathrm{~s}, 3 \mathrm{H}), 1.14(\mathrm{~s}, 3 \mathrm{H}) .{ }^{13} \mathrm{C}\left\{{ }^{1} \mathrm{H}\right\} \mathrm{NMR}$ $\left(126 \mathrm{MHz} \mathrm{CDCl}_{3}\right) \delta 143.0,139.2,138.9,137.6,137.6,137.3,137.2,135.3,134.3,130.9,129.2$, 128.7, 128.7, 128.6, 128.4, 127.9, 127.6 (q, $J=280.9 \mathrm{~Hz}) 125.9,121.7,45.8(\mathrm{q}, J=27.8 \mathrm{~Hz}), 22.7$ (q, $J=3.2 \mathrm{~Hz}), 21.2,20.9,19.9,19.6,18.4 .{ }^{19} \mathrm{~F} \mathrm{NMR}\left(376.5 \mathrm{MHz}, \mathrm{CDCl}_{3}, \mathrm{CF}_{3} \mathrm{CO}_{2} \mathrm{H}\right.$ - ext. st.): $\delta$ (ppm) $-68.4\left(\mathrm{~s}, 3 \mathrm{~F}\right.$ ). HRMS (APPI ${ }^{+}$-Orbitrap) $m / z$ : [M] ${ }^{+}$. Calcd for $\mathrm{C}_{28} \mathrm{H}_{29} \mathrm{~F}_{3}$ 422.2216; Found 422.2217 (0.4 ppm).

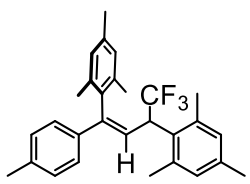

(Z)-2,2'-(4,4,4-Trifluoro-1-(p-tolyl)but-1-ene-1,3-diyl)bis(1,3,5trimethylbenzene) (6b) was prepared according to general procedure E from 1,1,1-trifluoro-4-( $p$-tolyl)but-3-yn-2-ol $(53 \mathrm{mg}, 0.25 \mathrm{mmol})$ and mesitylene $(105 \mu \mathrm{L}, 0.75 \mathrm{mmol})$, with $2.2 \mu \mathrm{L}(0.025 \mathrm{mmol})$ of triflic acid, in $0.125 \mathrm{~mL}$ of HFIP $\left(16 \mathrm{~h}, 50^{\circ} \mathrm{C}\right)$. Purification by flash column chromatography over silica (petroleum ether) gave $84 \mathrm{mg}\left(77 \%\right.$ yield) of white solid. Mp: $90-91{ }^{\circ} \mathrm{C} .{ }^{1} \mathrm{H}$ NMR $(400 \mathrm{MHz}$, $\left.\mathrm{CDCl}_{3}\right): \delta(\mathrm{ppm}) 7.16(\mathrm{~d}, J=8.3 \mathrm{~Hz}, 2 \mathrm{H}), 7.08(\mathrm{~d}, J=8.2 \mathrm{~Hz}, 2 \mathrm{H}), 6.95(\mathrm{~s}, 1 \mathrm{H}), 6.87(\mathrm{~d}, J=8.7$ $\mathrm{Hz}, 1 \mathrm{H}), 6.76(\mathrm{~s}, 1 \mathrm{H}), 6.65(\mathrm{~d}, J=4.5 \mathrm{~Hz}, 2 \mathrm{H}), 4.37-4.27(\mathrm{~m}, 1 \mathrm{H}), 2.50(\mathrm{~s}, 3 \mathrm{H}), 2.31(\mathrm{~s}, 3 \mathrm{H}), 2.28$ $(\mathrm{s}, 3 \mathrm{H}), 2.20(\mathrm{~s}, 3 \mathrm{H}), 2.17(\mathrm{~s}, 3 \mathrm{H}), 1.39(\mathrm{~s}, 3 \mathrm{H}), 1.13(\mathrm{~s}, 3 \mathrm{H}) \cdot{ }^{13} \mathrm{C}\left\{{ }^{1} \mathrm{H}\right\} \mathrm{NMR}\left(100 \mathrm{MHz}, \mathrm{CDCl}_{3}\right) \delta$ $142.8,139.2$, 137.8, 137.6, 137.5, 137.1, 136.0, 135.3, 134.5, 130.9, 129.4 (2C), 129.1, 128.6, 
128.4, $127.6(\mathrm{q}, J=281.2 \mathrm{~Hz}), 125.8(2 \mathrm{C}), 120.7,45.8$ (q, $J=27.8 \mathrm{~Hz}), 22.7(\mathrm{q}, J=3.1 \mathrm{~Hz}), 21.3$, 21.2, 20.9, 19.9, 19.6, 18.3. ${ }^{19} \mathrm{~F} \mathrm{NMR}\left(376.5 \mathrm{MHz}, \mathrm{CDCl}_{3}, \mathrm{CF}_{3} \mathrm{CO}_{2} \mathrm{H}\right.$ - ext. st.): $\delta$ (ppm) -68.3 (s, 3F). HRMS (APPI ${ }^{+}$-Orbitrap) $m / z$ : [M] ${ }^{+}$. Calcd for $\mathrm{C}_{29} \mathrm{H}_{31} \mathrm{~F}_{3}$ 436.2372; Found 436.2377 (1.1 ppm).

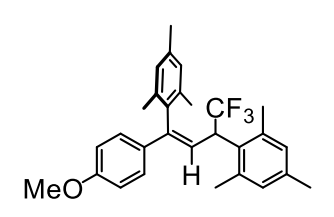

(Z)-2,2'-(4,4,4-Trifluoro-1-(4-methoxyphenyl)but-1-ene-1,3-diyl)bis(1,3,5trimethylbenzene) (6c) was prepared according to general procedure $\mathrm{E}$ from 1,1,1-trifluoro-4-(4-methoxyphenyl)but-3-yn-2-ol (58 mg, $0.25 \mathrm{mmol})$ and mesitylene $(105 \mu \mathrm{L}, 0.75 \mathrm{mmol})$, with $2.2 \mu \mathrm{L}(0.025 \mathrm{mmol})$ of triflic acid, in $0.125 \mathrm{~mL}$ of HFIP $\left(16 \mathrm{~h}, 50^{\circ} \mathrm{C}\right)$. Purification by flash column chromatography over silica (petroleum ether/EtOAc 98:2) gave $91 \mathrm{mg}$ (81\% yield) of colorless oil. ${ }^{1} \mathrm{H}$ NMR (400 $\left.\mathrm{MHz}, \mathrm{CDCl}_{3}\right): \delta(\mathrm{ppm}) 7.22(\mathrm{~d}, J=8.8 \mathrm{~Hz}, 2 \mathrm{H}), 6.98(\mathrm{~s}, 1 \mathrm{H}), 6.83(\mathrm{~d}, J=8.5 \mathrm{~Hz}, 2 \mathrm{H}), 6.79(\mathrm{~d}, J$ $=6.9 \mathrm{~Hz}, 2 \mathrm{H}), 6.68(\mathrm{~d}, J=5.2 \mathrm{~Hz}, 2 \mathrm{H}), 4.40-4.26(\mathrm{~m}, 1 \mathrm{H}), 3.81(\mathrm{~s}, 3 \mathrm{H}), 2.53(\mathrm{~s}, 3 \mathrm{H}), 2.31(\mathrm{~s}, 3 \mathrm{H})$, $2.23(\mathrm{~s}, 3 \mathrm{H}), 2.20(\mathrm{~s}, 3 \mathrm{H}), 1.42(\mathrm{~s}, 3 \mathrm{H}), 1.17(\mathrm{~s}, 3 \mathrm{H}) .{ }^{13} \mathrm{C}\left\{{ }^{1} \mathrm{H}\right\} \mathrm{NMR}\left(100 \mathrm{MHz}, \mathrm{CDCl}_{3}\right) \delta 159.5$, $142.4,139.2$, 137.6, 137.5, 137.1 (q, $J=2.6 \mathrm{~Hz}), 135.2,134.6,131.5,130.9,129.1,128.6,128.4$, 127.7 (q, $J=281.1 \mathrm{~Hz}$ ), 127.1 (2C), 119.6, 114.0 (2C), 55.4, 45.8 (q, $J=27.7 \mathrm{~Hz}), 21.2,20.9$, 19.9, 19.6, 18.3. ${ }^{19} \mathrm{~F}$ NMR (376.5 MHz, $\mathrm{CDCl}_{3}, \mathrm{CF}_{3} \mathrm{CO}_{2} \mathrm{H}$ - ext. st.): $\delta$ (ppm) -67.4 (s, 3F). HRMS (APPI+-Orbitrap) $\mathrm{m} / z$ : [M] ${ }^{+}$. Calcd for $\mathrm{C}_{29} \mathrm{H}_{31} \mathrm{OF}_{3} 452.2322$; Found 452.2323 (0.3 ppm).

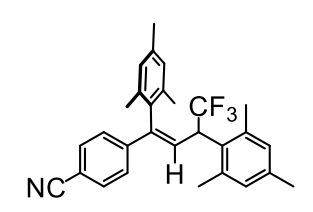

(Z)-4-(4,4,4-Trifluoro-1,3-dimesitylbut-1-en-1-yl)benzonitrile (6d) was prepared according to general procedure E from 4-(4,4,4-trifluoro-3hydroxybut-1-yn-1-yl)benzonitrile $(56 \mathrm{mg}, 0.25 \mathrm{mmol})$ and mesitylene (105 $\mu \mathrm{L}, 0.75 \mathrm{mmol})$, with $2.2 \mu \mathrm{L}(0.025 \mathrm{mmol})$ of triflic acid, in $0.125 \mathrm{~mL}$ of HFIP $\left(88 \mathrm{~h}, 100{ }^{\circ} \mathrm{C}\right)$. Purification by flash column chromatography over silica (petroleum ether/EtOAc 98:2) gave $25 \mathrm{mg}$ (22\% yield) of colorless oil. ${ }^{1} \mathrm{H}$ NMR (400 MHz, $\left.\mathrm{CDCl}_{3}\right): \delta(\mathrm{ppm}) 7.56(\mathrm{~d}, J=8.5 \mathrm{~Hz}, 2 \mathrm{H}), 7.35(\mathrm{~d}, J=8.5 \mathrm{~Hz}, 2 \mathrm{H}), 7.06(\mathrm{~d}, J=8.6 \mathrm{~Hz}, 1 \mathrm{H}), 6.98$ $(\mathrm{s}, 1 \mathrm{H}), 6.77(\mathrm{~s}, 1 \mathrm{H}), 6.67(\mathrm{~d}, J=8.6 \mathrm{~Hz}, 2 \mathrm{H}), 4.40-4.30(\mathrm{~m}, 1 \mathrm{H}), 2.48(\mathrm{~s}, 3 \mathrm{H}), 2.29(\mathrm{~s}, 3 \mathrm{H}), 2.20$ $(\mathrm{s}, 3 \mathrm{H}), 2.14(\mathrm{~s}, 3 \mathrm{H}), 1.40(\mathrm{~s}, 3 \mathrm{H}), 1.10(\mathrm{~s}, 3 \mathrm{H}) .{ }^{13} \mathrm{C}\left\{{ }^{1} \mathrm{H}\right\} \mathrm{NMR}\left(100 \mathrm{MHz}, \mathrm{CDCl}_{3}\right) \delta(\mathrm{ppm}) 143.3$, $141.8,139.3,138.0,137.6,137.5,137.4,135.2$, 133.0, 132.6, 131.0, 129.3, 128.9, 128.7, 128.0, $127.8(\mathrm{q}, J=280.7 \mathrm{~Hz}), 126.4,125.4,119.0,111.3,46.0(\mathrm{q}, J=28.0 \mathrm{~Hz}), 22.7(\mathrm{q}, J=3.8 \mathrm{~Hz})$, 21.2, 20.9, 19.8, 19.6, 18.3. ${ }^{19} \mathrm{~F} \mathrm{NMR} \mathrm{(376.5} \mathrm{MHz,} \mathrm{CDCl}_{3}, \mathrm{CF}_{3} \mathrm{CO}_{2} \mathrm{H}$ - ext. st.): $\delta$ (ppm) -67.3 (s, 3F). HRMS (APPI ${ }^{+}$-Orbitrap) $m / z$ : [M] $]^{+}$. Calcd for $\mathrm{C}_{29} \mathrm{H}_{28} \mathrm{NF}_{3} 447.2168$; Found $447.2172(0.8$ ppm).

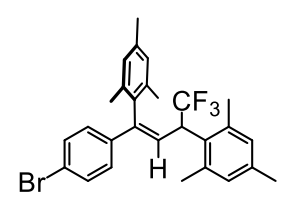

(Z)-2,2'-(1-(4-Bromophenyl)-4,4,4-trifluorobut-1-ene-1,3-diyl)bis(1,3,5trimethylbenzene) (6e) was prepared according to general procedure E from 4(4-bromophenyl)-1,1,1-trifluorobut-3-yn-2-ol (70 $\mathrm{mg}, 0.25 \mathrm{mmol})$ and mesitylene $(105 \mu \mathrm{L}, 0.75 \mathrm{mmol})$, with $2.2 \mu \mathrm{L}(0.025 \mathrm{mmol})$ of triflic acid, in $0.125 \mathrm{~mL}$ of HFIP $\left(16 \mathrm{~h}, 50{ }^{\circ} \mathrm{C}\right)$. Purification by flash column chromatography over silica (petroleum ether) gave $77 \mathrm{mg}$ (61\% yield) of colorless oil. ${ }^{1} \mathrm{H}$ NMR $\left(400 \mathrm{MHz}, \mathrm{CDCl}_{3}\right)$ : $\delta(\mathrm{ppm}) 7.40(\mathrm{~d}, J=8.6 \mathrm{~Hz}, 2 \mathrm{H}), 7.13(\mathrm{~d}, J=8.6 \mathrm{~Hz}, 2 \mathrm{H}), 6.96(\mathrm{~s}, 1 \mathrm{H}), 6.92(\mathrm{~d}, J=8.7 \mathrm{~Hz}, 1 \mathrm{H})$, $6.77(\mathrm{~s}, 1 \mathrm{H}), 6.67(\mathrm{~d}, J=6.1 \mathrm{~Hz}, 2 \mathrm{H}), 4.37-4.27(\mathrm{~m}, 1 \mathrm{H}), 2.48(\mathrm{~s}, 3 \mathrm{H}), 2.29(\mathrm{~s}, 3 \mathrm{H}), 2.21(\mathrm{~s}, 3 \mathrm{H})$, $2.16(\mathrm{~s}, 3 \mathrm{H}), 1.40(\mathrm{~s}, 3 \mathrm{H}), 1.13(\mathrm{~s}, 3 \mathrm{H}) .{ }^{13} \mathrm{C}\left\{{ }^{1} \mathrm{H}\right\}$ NMR $\left(100 \mathrm{MHz}, \mathrm{CDCl}_{3}\right): \delta(\mathrm{ppm}) 142.3,139.4$, $138.0,137.8,137.7,137.5,135.4,134.5,133.9,132.0,131.1,129.4,128.9,128.7,123.0(\mathrm{q}, J=$ $281.2 \mathrm{~Hz}), 127.7,126.0,123.1,122.2,46.1$ (q, $J=27.8 \mathrm{~Hz}), 22.9,21.5,21.0,20.1,19.8,18.5 .{ }^{19} \mathrm{~F}$ 
NMR (376.5 MHz, $\mathrm{CDCl}_{3}, \mathrm{CF}_{3} \mathrm{CO}_{2} \mathrm{H}$ - ext. st.): $\delta$ (ppm) -67.3 (s, 3F). HRMS (APPI+-Orbitrap) $m / z:[\mathrm{M}]^{+}$. Calcd for $\mathrm{C}_{28} \mathrm{H}_{28}{ }^{79} \mathrm{BrF}_{3}$ 500.1321; Found 500.1331 (1.9 ppm).

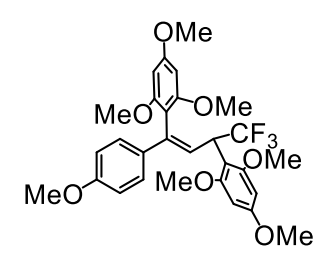

(Z)-2,2'-(4,4,4-Trifluoro-1-(4-methoxyphenyl)but-1-ene-1,3-diyl)bis(1,3,5trimethoxybenzene) (6f) was prepared according to general procedure $\mathrm{E}$ from 1,1,1-trifluoro-4-(4-methoxyphenyl)but-3-yn-2-ol $(58 \mathrm{mg}, 0.25 \mathrm{mmol})$ and 1,3,5-trimethoxybenzene (116 mg, $0.75 \mathrm{mmol})$, with $2.2 \mu \mathrm{L}(0.025 \mathrm{mmol})$ of triflic acid, in $0.125 \mathrm{~mL}$ of HFIP $\left(16 \mathrm{~h}, 50^{\circ} \mathrm{C}\right)$. Purification by flash column chromatography over silica (petroleum ether/EtOAc 8:2) gave $100 \mathrm{mg}$ ( $73 \%$ yield) of white solid. Mp: $128-129^{\circ} \mathrm{C} .{ }^{1} \mathrm{H}$ NMR $\left(400 \mathrm{MHz}, \mathrm{CDCl}_{3}\right): \delta(\mathrm{ppm}) 7.24(\mathrm{~d}, J=8.9 \mathrm{~Hz}, 2 \mathrm{H}), 6.79$ (dd, $J=$ 21.3, $8.5 \mathrm{~Hz}, 3 \mathrm{H}), 6.20(\mathrm{~d}, J=2.1 \mathrm{~Hz}, 1 \mathrm{H}), 6.08(\mathrm{~s}, 1 \mathrm{H}), 5.90(\mathrm{~s}, 1 \mathrm{H}), 5.87(\mathrm{~d}, J=2.1 \mathrm{~Hz}, 1 \mathrm{H})$, $4.60-4.49(\mathrm{~m}, 1 \mathrm{H}), 3.82(\mathrm{~s}, 6 \mathrm{H}), 3.78(\mathrm{~s}, 3 \mathrm{H}), 3.76(\mathrm{~s}, 6 \mathrm{H}), 3.43(\mathrm{~s}, 3 \mathrm{H}), 3.07(\mathrm{~s}, 3 \mathrm{H}) .{ }^{13} \mathrm{C}\left\{{ }^{1} \mathrm{H}\right\}$ NMR $\left(100 \mathrm{MHz}, \mathrm{CDCl}_{3}\right): \delta(\mathrm{ppm}) 161.1,160.8,159.2,159.0,158.3,135.5,133.9,127.8$ (q, $J=$ $280.9 \mathrm{~Hz}), 127.3,123.2,113.7,109.5,105.9,91.6,90.5,90.4,90.2,56.4,56.2,55.7,55.6(2 \mathrm{C})$, 55.5, 55.2, 30.2 (q, $J=27.8 \mathrm{~Hz}) .{ }^{19} \mathrm{~F}$ NMR (376.5 MHz, $\mathrm{CDCl}_{3}, \mathrm{CF}_{3} \mathrm{CO}_{2} \mathrm{H}-$ ext. st.): $\delta$ (ppm) 68.2 (s, 3F). HRMS (ESI-Orbitrap) $m / z$ : $[\mathrm{M}+\mathrm{H}]^{+}$Calcd for $\mathrm{C}_{29} \mathrm{H}_{32} \mathrm{O}_{7} \mathrm{~F}_{3} 549.2095$; Found 549.2114 (3.6 ppm).

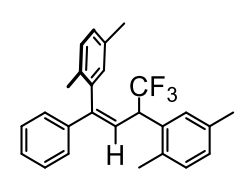

(Z)-2,2'-(4,4,4-Trifluoro-1-phenylbut-1-ene-1,3-diyl)bis(1,4-dimethylbenzene) (6g) was prepared according to general procedure $\mathrm{E}$ from 1,1,1-trifluoro-4phenylbut-3-yn-2-ol ( $50 \mu \mathrm{L}, 0.25 \mathrm{mmol})$ and $p$-xylene $(92 \mu \mathrm{L}, 0.75 \mathrm{mmol})$, with $2.2 \mu \mathrm{L}(0.025 \mathrm{mmol})$ of triflic acid, in $0.125 \mathrm{~mL}$ of $\operatorname{HFIP}\left(16 \mathrm{~h}, 50^{\circ} \mathrm{C}\right)$. Purification by flash column chromatography over silica (petroleum ether) gave $48 \mathrm{mg}$ ( $49 \%$ yield) of colorless oil which was isolated as a 6:4 mixture of stereoisomers as determined by ${ }^{1} \mathrm{H}$ NMR. ${ }^{1} \mathrm{H}$ NMR (400 $\left.\mathrm{MHz}, \mathrm{CDCl}_{3}\right): \delta(\mathrm{ppm}) 6.69(\mathrm{~d}, J=9.7 \mathrm{~Hz}, 1 \mathrm{H}$, major), $6.68(\mathrm{~d}, J=9.7 \mathrm{~Hz}, 1 \mathrm{H}$, minor), $6,36(\mathrm{~s}$, $1 \mathrm{H}$, minor), 4.39-4.22 (m, 1H, minor), 4.15-4.01 (m, 1H, major), 2.41 (s, 3H, minor), 2.34 (s, 6H, major), 2.16 (s, 3H, major), 2.06 (s, 3H, major), 1.78 (s, 3H, minor), 1.68 (s, 3H, minor), 1.45 (s, $3 \mathrm{H}$, minor). ${ }^{13} \mathrm{C}\left\{{ }^{1} \mathrm{H}\right\}$ NMR $\left(101 \mathrm{MHz}, \mathrm{CDCl}_{3}\right) \delta 146.1,145.8,140.0,139.8,138.0,137.8,135.7$, 135.7, 135.3, 134.5, 134.0, 133.8, 133.7, 133.6, 133.3, 130.5, 130.4, 130.4, 130.3, 130.2, 129.7, $129.2,128.8,128.7,128.6,128.5,128.0,126.7,126.6,45.2$ (q, $J=27.4 \mathrm{~Hz}), 44.8$ (q, $J=27.4 \mathrm{~Hz})$, 21.3, 21.2, 20.9, 19.1, 18.5, 18.5. (mixture of two rotamers). ${ }^{19} \mathrm{~F} \mathrm{NMR}\left(376.5 \mathrm{MHz}, \mathrm{CDCl}_{3}\right.$, $\mathrm{CF}_{3} \mathrm{CO}_{2} \mathrm{H}$ - ext. st.): $\delta$ (ppm) -69.2 (s, 2.2F - minor), -69.5 (s, 3F - major). HRMS (APPI ${ }^{+}$ Orbitrap) $m / z$ : $[\mathrm{M}]^{+}$. Calcd for $\mathrm{C}_{26} \mathrm{H}_{25} \mathrm{~F}_{3}$ 394.1908; Found 394.1902 (-1.7 ppm).

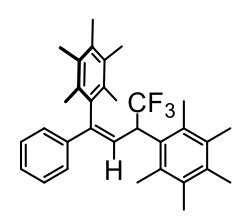

(Z)-6,6'-(4,4,4-Trifluoro-1-phenylbut-1-ene-1,3-diyl)bis(1,2,3,4,5-pentamethylbenzene) (6h) was prepared according to general procedure $\mathrm{E}$ from 1,1,1trifluoro-4-phenylbut-3-yn-2-ol ( $50 \mu \mathrm{L}, 0.25 \mathrm{mmol})$ and pentamethylbenzene $(111$ $\mathrm{mg}, 0.75 \mathrm{mmol})$, with $2.2 \mu \mathrm{L}(0.025 \mathrm{mmol})$ of triflic acid, in $0.125 \mathrm{~mL}$ of HFIP (16 $\mathrm{h}, 50{ }^{\circ} \mathrm{C}$ ). Purification by flash column chromatography over silica (petroleum ether) gave $114 \mathrm{mg}$ ( $95 \%$ yield) of white solid. Mp: $183-185^{\circ} \mathrm{C} .{ }^{1} \mathrm{H}$ NMR (400 MHz, $\left.\mathrm{CDCl}_{3}\right)$ : $\delta$ (ppm) 7.31-7.24 (m, 5H), $6.94(\mathrm{~d}, J=7.4 \mathrm{~Hz}, 1 \mathrm{H}), 4.53-4.43(\mathrm{~m}, 1 \mathrm{H}), 2.41(\mathrm{~s}, 3 \mathrm{H}), 2.27(\mathrm{~s}, 3 \mathrm{H})$, $2.25(\mathrm{~s}, 3 \mathrm{H}), 2.21(\mathrm{~s}, 3 \mathrm{H}), 2.20(\mathrm{~s}, 3 \mathrm{H}), 2.16(\mathrm{~s}, 3 \mathrm{H}), 2.02(\mathrm{~s}, 3 \mathrm{H}), 1.82(\mathrm{~s}, 3 \mathrm{H}), 1.29(\mathrm{~s}, 3 \mathrm{H}), 0.84$ (s, $3 \mathrm{H}) .{ }^{13} \mathrm{C}\left\{{ }^{1} \mathrm{H}\right\}$ NMR $\left(100 \mathrm{MHz}, \mathrm{CDCl}_{3}\right): \delta$ (ppm) 144.4, 140.0, 135.1, 134.8, 134.4, 134.0, 
133.7, 133.4, 133.2, 132.9, 132.7, 132.4, 130.3, 129.7, 128.8, 127.8, 127.8 (q, $J=280.5 \mathrm{~Hz}), 126.2$, $122.8,46.2(\mathrm{q}, J=27.9 \mathrm{~Hz}), 19.9,19.8,17.5,17.4,17.3,17.1,16.9,16.8,16.2,15.9 .{ }^{19} \mathrm{~F}$ NMR (376.5 MHz, $\mathrm{CDCl}_{3}, \mathrm{CF}_{3} \mathrm{CO}_{2} \mathrm{H}$ - ext. st.): $\delta$ (ppm) -66.3 (s, 3F). HRMS (APPI+-Orbitrap) $m / z$ : $[\mathrm{M}+\mathrm{H}]^{+}$Calcd for $\mathrm{C}_{32} \mathrm{H}_{38} \mathrm{~F}_{3} 479.2920$; Found 479.2923 (0.6 ppm).

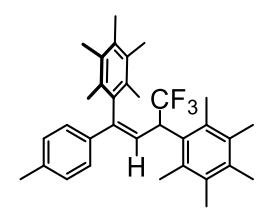

(Z)-6,6'-(4,4,4-Trifluoro-1-(p-tolyl)but-1-ene-1,3-diyl)bis(1,2,3,4,5pentamethylbenzene) (6i) was prepared according to general procedure $\mathrm{E}$ from 1,1,1-trifluoro-4-( $p$-tolyl)but-3-yn-2-ol $\quad(53 \quad \mathrm{mg}, \quad 0.25 \quad \mathrm{mmol}) \quad$ and pentamethylbenzene $(111 \mathrm{mg}, 0.75 \mathrm{mmol})$, with $2.2 \mu \mathrm{L}(0.025 \mathrm{mmol})$ of triflic acid, in $0.125 \mathrm{~mL}$ of HFIP $\left(16 \mathrm{~h}, 50{ }^{\circ} \mathrm{C}\right)$. Purification by flash column chromatography over silica (petroleum ether) gave $108 \mathrm{mg}(88 \%$ yield) of white solid. Mp: 206$209^{\circ} \mathrm{C} .{ }^{1} \mathrm{H}$ NMR $\left(400 \mathrm{MHz}, \mathrm{CDCl}_{3}\right): \delta(\mathrm{ppm}) 7.19(\mathrm{~d}, J=8.2 \mathrm{~Hz}, 2 \mathrm{H}), 7.09(\mathrm{~d}, J=8.1 \mathrm{~Hz}, 2 \mathrm{H})$, $6.89(\mathrm{~d}, J=7.4 \mathrm{~Hz}, 1 \mathrm{H}), 4.52-4.41(\mathrm{~m}, 1 \mathrm{H}), 2.39(\mathrm{~s}, 3 \mathrm{H}), 2.33(\mathrm{~s}, 3 \mathrm{H}), 2.27(\mathrm{~s}, 3 \mathrm{H}), 2.21(\mathrm{~s}, 3 \mathrm{H})$, 2.19 (s, 3H), 2.15 (s, 3H), $2.02(\mathrm{~s}, 3 \mathrm{H}), 1.82(\mathrm{~s}, 3 \mathrm{H}), 1.29$ (s, 3H), 0.84 (s, 3H), 0.09 (s, 3H). ${ }^{13} \mathrm{C}\left\{{ }^{1} \mathrm{H}\right\}$ NMR $\left(100 \mathrm{MHz}, \mathrm{CDCl}_{3}\right): \delta(\mathrm{ppm}) 144.2,137.7,137.2,135.1,134.9,134.4,133.9,133.7$, $133.4,133.2,132.8,132.7,132.0,130.3,129.8,129.5,127.8$ (q, $J=281.1 \mathrm{~Hz}), 126.1,121.8,46.2$ $(\mathrm{q}, J=27.8 \mathrm{~Hz}), 21.4,19.9,19.8,17.5,17.4,17.2,17.0,16.9,16.8,16.2,15.9 .{ }^{19} \mathrm{~F}$ NMR $(376.5$ $\mathrm{MHz}, \mathrm{CDCl}_{3}, \mathrm{CF}_{3} \mathrm{CO}_{2} \mathrm{H}$ - ext. st.): $\delta(\mathrm{ppm})-66.3$ (s, 3F). HRMS (APPI ${ }^{+}$-Orbitrap) $m / z$ : $[\mathrm{M}]^{+}$. Calcd for $\mathrm{C}_{33} \mathrm{H}_{39} \mathrm{~F}_{3}$ 492.2998; Found 492.3005 (1.2 ppm).

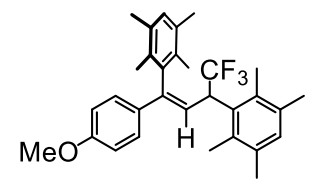

(Z)-3,3'-(4,4,4-Trifluoro-1-(4-methoxyphenyl)but-1-ene-1,3-diyl)bis(1,2,4,5tetramethylbenzene) $(\mathbf{6 j})$ was prepared according to general procedure $E$ from 1,1,1-trifluoro-4-(4-methoxyphenyl)but-3-yn-2-ol (58 $\mathrm{mg}, 0.25 \mathrm{mmol}$ ) and durene (102 $\mathrm{mg}, 0.75 \mathrm{mmol})$, with $2.2 \mu \mathrm{L}(0.025 \mathrm{mmol})$ of triflic acid, in 0.125 $\mathrm{mL}$ of HFIP $\left(16 \mathrm{~h}, 50^{\circ} \mathrm{C}\right)$. Purification by flash column chromatography over silica (petroleum ether/EtOAc 98:2) gave $65 \mathrm{mg}$ (54\% yield) of white solid. Mp: $144-146{ }^{\circ} \mathrm{C} .{ }^{1} \mathrm{H}$ NMR $(400 \mathrm{MHz}$, $\left.\mathrm{CDCl}_{3}\right): \delta(\mathrm{ppm}) 7.19(\mathrm{~d}, J=8.8 \mathrm{~Hz}, 2 \mathrm{H}), 6.89(\mathrm{~s}, 1 \mathrm{H}), 6.83(\mathrm{~d}, J=7.9 \mathrm{~Hz}, 2 \mathrm{H}), 6.81(\mathrm{~d}, J=8.8$ $\mathrm{Hz}, 2 \mathrm{H}), 4.52-4.35(\mathrm{~m}, 1 \mathrm{H}), 3.78(\mathrm{~s}, 3 \mathrm{H}), 2.36(\mathrm{~s}, 3 \mathrm{H}), 2.27(\mathrm{~s}, 3 \mathrm{H}), 2.15(\mathrm{~s}, 3 \mathrm{H}), 2.11(\mathrm{~s}, 3 \mathrm{H})$, $2.06(\mathrm{~s}, 3 \mathrm{H}), 1.88(\mathrm{~s}, 3 \mathrm{H}), 1.22(\mathrm{~s}, 3 \mathrm{H}), 0.89(\mathrm{~s}, 3 \mathrm{H}) .{ }^{13} \mathrm{C}\left\{{ }^{1} \mathrm{H}\right\} \mathrm{NMR}\left(100 \mathrm{MHz}, \mathrm{CDCl}_{3}\right): \delta(\mathrm{ppm})$ 159.6, 143.4, 137.7, 135.7, 134.8, 134.4, 134.1, 133.9, 133.8, 133.4, 133.3, 131.9, 131.2, 130.6, $130.4,127.5$ (q, $J=281.1 \mathrm{~Hz}), 127.2,119.9,110.2,55.4,45.9$ (q, $J=27.8 \mathrm{~Hz}), 21.4,21.1,20.5$, 19.9, 19.7, 18.6, 16.1, 15.4, 14.9. ${ }^{19} \mathrm{~F} \mathrm{NMR}\left(376.5 \mathrm{MHz}, \mathrm{CDCl}_{3}, \mathrm{CF}_{3} \mathrm{CO}_{2} \mathrm{H}-\right.$ ext. st.): $\delta$ (ppm) 66.3 (s, 3F). HRMS (APPI ${ }^{+}$-Orbitrap) $m / z$ : [M] ${ }^{+}$. Calcd for $\mathrm{C}_{31} \mathrm{H}_{35} \mathrm{OF}_{3} 480.2635$; Found 480.2636 (0.4 ppm).

\section{ASSOCIATED CONTENT}

The Supporting Information is available free of charge on the ACS Publications website at DOI: $10.1021 / \mathrm{xxxxxxxx}$.

X-ray crystallography data of $1 \mathrm{~s}(\mathrm{CIF})$ and $\mathbf{6 a}(\mathrm{CIF})$.

${ }^{1} \mathrm{H}$ and ${ }^{13} \mathrm{C}\left\{{ }^{1} \mathrm{H}\right\}$, and ${ }^{19} \mathrm{~F}$ NMR spectra for the products (PDF). 


\title{
AUTHOR INFORMATION
}

\section{Corresponding Author}

\author{
*E-mail: moran@unistra.fr
}

\section{ORCID}

Joseph Moran: 0000-0002-7851-6133

\section{Notes}

The authors declare no competing financial interest.

CCDC 1960543 and 1960571 contain the supplementary crystallographic data for this paper.

These data can be obtained free of charge from The Cambridge Crystallographic Data Centre.

\section{ACKNOWLEDGEMENTS}

J.M thanks the European Research Council (ERC) (grant agreement $n^{\circ}$ 639170) and the ANR LabEx "Chemistry of Complex Systems" (ANR-10-LABX-0026 CSC). F.N. thanks the Région Grand-Est for a fellowship. V.D.V thanks the French Ministry of Higher Education for a fellowship. J.Y. thanks the China Scholarship Council for a fellowship. The authors thank Dr. Lydia Karmazin and Dr. Corinne Bailly for X-ray crystallography data and Mr. Cyril Antheaume for HRMS data.

\section{REFERENCES}

${ }^{1}$ (a) Qian, H.; Huang, D.; Bi, Y.; Yan, G. 2-Propargyl Alcohols in Organic Synthesis. Adv. Synth. Cat. 2019. (b) Debleds, O.; Gaynon, E.; Vrancken, E.; Campagne, J.-M. Gold-catalyzed propargylic substitutions: Scope and synthetic developments. Beilstein J. Org. Chem. 2011, 7, 866-877.

${ }^{2}$ (a) Bandini, M.; Tragni, M. $\pi$-Activated alcohols: an emerging class of alkylating agents for catalytic FriedelCrafts reactions. Org. Biomol. Chem. 2009, 7, 1501-1507. (b) Rueping, M.; Nachtsheim, B. J. A review of new developments in the Friedel-Crafts alkylation - From green chemistry to asymmetric catalysis. Beilstein J. Org. Chem. 2010, 6, No. 6. (c) Kumar, R.; Van der Eycken, E. V. Recent approaches for C-C bond formation via direct dehydrative coupling strategies. Chem. Soc. Rev. 2013, 42, 1121-1146. (d) Naredla, R. R.; Klumpp, D. A. Contemporary Carbocation Chemistry: Applications in Organic Synthesis. Chem. Rev. 2013, 113, $6905-6948$.

(e) Baeza, A.; Nájera, C. Recent Advances in the Direct Nucleophilic Substitution of Allylic Alcohols through SN1Type Reactions. Synthesis 2014, 46, 25-34. (f) Dryzhakov, M.; Richmond, E.; Moran J.; Recent Advances in Direct Catalytic Dehydrative Substitution of Alcohols. Synthesis, 2016, 48, 935-959.

${ }^{3}$ Some of the recent reports include: (a) Kennedy-Smith, J. J.; Young, L. A.; Toste, F. D. Rhenium-Catalyzed Aromatic Propargylation. Org. Lett. 2004, 6, 1325-1327. (b) Georgy, M.; Boucard, V.; Campagne, J.-M. Gold(III)Catalyzed Nucleophilic Substitution of Propargylic Alcohols. J. Am. Chem. Soc. 2005, 127, 14180-14181. (c) Huang, G.-B.; Wang X.; Pan, Y.-M.; Wang, H. S.; Yao, G.-Y.; Zhang, Y. Atom-Economical Chemoselective Synthesis of 1,4-Enynes from Terminal Alkenes and Propargylic Alcohols Catalyzed by Cu(OTf)2. J. Org. Chem. 2013, 78, 2742- 
2745. (d) Ponra, S.; Gohain, M.; van Tonder, J. H.; Bezuidenhoudt, B. C. B. Al(OTf)3-Catalyzed Preparation of 4Hydroxy-3-propargylic -Coumarins and Subsequent Regioselective Cyclization towards Furo- or Pyrano[3,2c]coumarins. Synlett 2015, 26, 745-750.

${ }^{4}$ C-C bond forming reactions (a) Xu, C.-F.; Xu, M.; Yang, L.-Q.; Li, C.-Y. Synthesis of Allenes via Gold-Catalyzed Intermolecular Reaction of Propargylic Alcohols and Aromatic Compounds. J. Org. Chem. 2012, 77, 3010-3016. (b) Ricardo, C. L.; Mo, X.; McCubbin, J. A.; Hall, D. G. A Surprising Substituent Effect Provides a Superior Boronic Acid Catalyst for Mild and Metal-Free Direct Friedel-Crafts Alkylations and Prenylations of Neutral Arenes. Chem. Eur. J. 2015, 21, 4218-4223. (c) Huang, K.; Sheng, G.; Lu, P.; Wang, Y. From 1-Sulfonyl-4-aryl-1,2,3-triazoles to 1Allenyl-5-aryl-1,2,3-triazoles. J. Org. Chem. 2017, 82, 5294-5300.

${ }^{5}$ For generation of $\alpha$-trifluoromethyl cations via tosylates, triflates, bromides and $p$-nitrobenzoates, see: (a) Allen, A. D.; Ambridge, I. C.; Che, C.; Michael, H.; Muir, R. J.; Tidwell, T. T. Solvolysis of 1-aryl-2,2,2-trifluoroethyl sulfonates. Kinetic and stereochemical effects in the generation of highly electron-deficient carbocations. $J$. Am. Chem. Soc. 1983, 105, 2343-2350. (b) Allen, A. D.; Girdhir, R.; Jansen, M. P.; Mayo, J. D.; Tidwell, T. T. Solvolysis of 1-(1-naphthyl)- and 1-(9-anthryl)-2,2,2-trifluoroethyl sulfonates. J. Org. Chem. 1986, 51, 1324-1329. (c) Richard, J. P. Surprisingly small effect of an alpha-trifluromethyl-for-alpha-methyl substitution on 1-(4-methoxyphenyl)ethyl cation reactivity. J. Am. Chem. Soc. 1986, 108, 6819-6820. (d) Richard, J. P. Desolvation-limited reactions of amines with the 1-(4-methylthiophenyl)-2,2,2-trifluoromethyl carbocation. J. Chem. Soc.; Chem. Commun. 1987, 1768-1769. (e) Richard, J. P. The extraordinarily long lifetimes and other properties of highly destabilized ring-substituted 1phenyl-2,2,2-trifluoroethyl carbocations. J. Am. Chem. Soc. 1989, 111, 1455-1465. (f) Richard, J. P. Reduction of the 1-(4-thiomethylphenyl)-2,2,2-trifluoroethyl carbocation by sodium sulphite. Tetrahedron Lett. 1989, 30, 23-26. (g) Kwong-Chip, J.-M.; Tidwell, T. T. Solvolytic reactivity of 1-(1-methyl-2-pyrrolyl)-2,2,2-trifluoroethyl pnitrobenzoate. Tetrahedron Lett. 1989, 30, 1319-1322.

${ }^{6}$ Bergmann, E. D.; Pelchowicz, Z.; Shani, A. Some Fluorine Containing D.D.T. Analogues. Isr. J. Chem. 1963, 1, 129-135.

${ }^{7}$ Interestingly, Vasilyev et al. published analogous transformation of $\mathrm{CF}_{3}$-allylic alcohols with $\mathrm{FeCl}_{3}$ (or $\mathrm{FSO}_{3} \mathrm{H}$ ) as catalyst in $\mathrm{CH}_{2} \mathrm{Cl}_{2}$, giving $\mathrm{CF}_{3}$-alkenes and $\mathrm{CF}_{3}$-indanes, see: Kazakova, A. N.; Iakovenko, R. O.; Boyarskaya, I. A.; Nenajdenko, V. G.; Vasilyev, A. V. Acid-Promoted Reaction of Trifluoromethylated Allyl Alcohols with Arenes. Stereoselective Synthesis of $\mathrm{CF}_{3}-\mathrm{Alkenes}$ and $\mathrm{CF}_{3}$-Indanes. J. Org. Chem. 2015, 80, 9506-9517.

${ }^{8}$ Schlosser, M. Parametrization of Substituents: Effects of Fluorine and Other Heteroatoms on $\mathrm{OH}, \mathrm{NH}$, and CH Acidities. Angew. Chem. Int. Ed. 1998, 110, 1496-1513

${ }^{9}$ Böhm, H.-J.; Banner, D.; Bendels, S.; Kansy, M.; Kuhn, B.; Müller, K.; Obst-Sander, U.; Stahl, M. Fluorine in Medicinal Chemistry. ChemBioChem 2004, 5, 637-643.

${ }^{10}$ (a) Colomer, I.; Chamberlain, A. E. R.; Haughey, M. B.; Donohoe, T. J. Hexafluoroisopropanol as a highly versatile solvent. Nat. Chem. Rev. 2017, 1, Article No. 88. (b) Pezacki J. P.; Shukla D.; Lusztyk J.; Warkentin, J. Lifetimes of Dialkylcarbocations Derived from Alkanediazonium Ions in Solution: Cyclohexadienyl Cations as Kinetic Probes for Cation Reactivity. J. Am. Chem. Soc. 1999, 121, 6589-6598.

${ }^{11}$ Vuković V. D.; Richmond E.; Wolf E.; Moran J. Catalytic Friedel-Crafts Reactions of Highly Electronically Deactivated Benzylic Alcohols. Angew. Chem. Int. Ed. 2017, 56, 3085-3089.

12 (a) Smart, B. E.; Fluorine substituent effects on bioactivity. J. Fluor. Chem. 2001, 109, 3-11. (b) Müller, K.; Faeh, C.; Diderich, F. Fluorine in pharmaceuticals: looking beyond intuition. Science, 2007, 317, 1881-1886. (c) Purser, S.; Moore, P. R.; Swallow, S.; Gouverneur, V. Fluorine in medicinal chemistry. Chem. Soc. Rev. 2008, 37, 320-330. (d) Zhu, W.; Wang, J.; Wang, S.; Gu, Z.; Aceña, J. L.; Izawa, K.; Liu, H.; Soloshonok, V. A. Recent advances in the trifluoromethylation methodology and new $\mathrm{CF}_{3}$-containing drugs. J. Fluor. Chem. 2014, 167, 37-54.

${ }^{13}$ Krause, N.; Hashmi, A. S. K.; Eds. Modern Allene Chemistry, Wiley-VCH: Weinheim, 2004; Vols. 1 and 2.

${ }^{14}$ (a) Wytanabe, Y.; Yamazaki, T. Facile Preparation of $\mathrm{CF}_{3}$-Containing 1-Bromoallenes. Synlett, 2009, 20, $3352-$ 3354. (b) Ambler, B. R.; Peddi, S.; Altman, R. A. Ligand-Controlled Regioselective Copper-Catalyzed Trifluoromethylation To Generate (Trifluoromethyl)allenes. Org. Lett. 2015, 17, 2506-2509

${ }^{15}$ From $\gamma-\mathrm{CF}_{3}$ propargyl alcohols: (a) Li J.-L.; Yang X.-J.; Jiang M.; Liu, J.-T. The preparation and cycloaddition reaction of 1-sulfonyl-1-trifluoromethyl allenes from prefunctionalized propargyl alcohols. Tetrahedron Lett. 2017, 58, 3377-3379. (b) Yamazaki, T.; Yamamoto, T.; Ichihara, R. Preparation of $\mathrm{CF}_{3}$-Containing 1,3-Di- and 1,1,3Trisubstituted Allenes. J. Org. Chem. 2006, 71, 6251-6253. (c) Konno, T.; Tanikawa, M.; Ishihara, T.; Yamanaka, H. Palladium-Catalyzed Coupling Reaction of Fluoroalkylated Propargyl Mesylates with Organozinc Reagents: Novel Synthesis of Optically Active Fluorine-Containing Trisubstituted Allenes. Chemistry Letters, 2000, 29(12), 13601361. (d) Konno, T.; Tanikawa, M.; Ishihara, T.; Yamanaka, H. Fluorine-Containing Allenylpalladium Complexes. Synthesis of Optically Active Fluoroalkylated Allenes and Furan Derivatives. Collect. Czech. Chem. Commun. 2002, 67, 1421-1435. (e) Boreux, A.; Lonca, G. H.; Riant, O.; Gagosz, F. Synthesis of Trifluoromethyl-allenes by Gold- 
Catalyzed Rearrangement of Propargyl Benzyl Ethers: By trifluoromethylation of alkynes. Org. Lett. 2016, 18, 5162 5165 (f) Ji, Y.-L.; Luo, J.-J.; Lin, J.-H.; Xiao, J.-C.; Gu, Y.-C. Cu-Catalyzed C-H Trifluoromethylation of 3-Arylprop1-ynes for the Selective Construction of Allenic $\mathrm{Csp}_{2}-\mathrm{CF}_{3}$ and Propargyl $\mathrm{Csp}_{3}-\mathrm{CF}_{3}$ Bonds: By trifluoromethylation of propargyl halides. Org. Lett. 2016, 18, 1000-1003. (g) Zhao, T. S. N.; Szabó, K. J. Trifluoromethylation of Propargylic Halides and Trifluoroacetates Using $\left(\mathrm{Ph}_{3} \mathrm{P}\right) 3 \mathrm{Cu}\left(\mathrm{CF}_{3}\right)$ Reagent: By trifluoromethylation of propagryl acetates. Org. Lett. 2012, 14, 3966-3969. (h) Ji, Y.-L.; Kong, J.-J.; Lin, J.-H.; Xiao, J.-C.; Gu, Y.-C. Copper-mediated trifluoromethylation of propargyl acetates leading to trifluoromethyl-allenes. Org. Biomol. Chem. 2014, 12, 29032906.

${ }^{16}$ Shimizu, M.; Higashi, M.; Takeda, Y.; Jiang, G.; Murai, M.; Hiyama, T. Novel Generation of 3,3,3-

Trifluoropropynyllithium and Transformation of the Carbonyl Adducts to Trifluoromethyl-Substituted Allenes.

Synlett 2007, 7, 1163-1165.

${ }^{17}$ Gassman, P. G.; Ray, J. A.; Wenthold, P. G.; Mickelson, J. W. Synthesis of perfluoroalkylated indenes. J. Org. Chem. 1991, 56, 5143-5146.

${ }^{18}$ (a) Radix-Large, S.; Kucharski, S.; Langlois, B. R. Trifluoromethylated Vinylic and Aromatic Compounds from $\alpha-$ (Trifluoro-methyl)allyl Alcohols. Synthesis, 2004, 3, 456-465. (b) Zhang, J.; Wu, H.-H.; Zhang, J. Enantioselective Phosphine-Catalyzed Allylic Alkylations of mix-Indene with MBH Carbonates. Org. Lett. 2017, 19, 6080-6083.

${ }^{19}$ Kazakova, A. N.; Iakovenko, R. O.; Boyarskaya, I. A.; Ivanov, A. Yu.; Avdontceva, M. S.; Zolotarev, A. A.; Panikorovsky, T. L.; Starova, G. L.; Nenajdenko, V. G.; Vasilyev, A. V. Brominated $\mathrm{CF}_{3}$-allyl alcohols as multicentered electrophiles in TfOH promoted reactions with arenes. Org. Chem. Front. 2017, 4, 255-265.

${ }^{20}$ Krause N.; Hashmi A. S. K. Modern Allene Chemistry, 2014, Vol. 2. Wiley-VCH

${ }^{21}$ Iakovenko, R. O.; Chicca, A.; Nieri, D.; Reynoso-Moreno, I.; Gertsch, J.; Krasavin, M.; Vasilyev, A. V. Synthesis of various arylated trifluoromethyl substituted indanes and indenes, and study of their biological activity. Tetrahedron 2019, 75, 624-632.

${ }^{22}$ Nursahedova, S. K.; Zerov, A. V.; Boyarskaya, I. A.; Grinenko, E. V.; Nenajdenko, V. G.; Vasilyev, A. V. HUSY zeolite-promoted reactions of trifluoromethylated propargyl alcohols with arenes: synthesis of $\mathrm{CF}_{3}$-indenes and DFT study of intermediate carbocations. Org. Biomol. Chem. 2019, 17, 1215-1224.

23 Pratap, R.; Ram, V. J. Natural and Synthetic Chromenes, Fused Chromenes, and Versatility of Dihydrobenzo[h]chromenes in Organic Synthesis. Chem. Rev. 2014, 114, 10476-10526.

24 (a) Wang, J. L.; Carter, J.; Kiefer, J. R.; Kurumbail, R. G.; Pawlitz, J. L.; Brown, D.; Hartmann, S. J.; Graneto, M. J.; Seibert, K.; Talley, J. J. The novel benzopyran class of selective cyclooxygenase-2 inhibitors-part I: the first clinical candidate. Bioorg. Med. Chem. Lett. 2010, 20, 7155-7158 and 7159-7163 and 7164-7168. (b) Xing, L.; Hamper, B. C.; Fletcher, T. R.; Wendling, J. M.; Carter, J.; Gierse, J. K.; Liao S. Structure-based parallel medicinal chemistry approach to improve metabolic stability of benzopyran COX-2 inhibitors. Bioorg. Med. Chem. Lett. 2011, 21, 993996. (c) Zhang, Y.; Tortorella, M. D.; Wang, Y.; Liu, J.; Tu, Z.; Liu, X.; Bai, Y.; Wen, D.; Lu, X.; Lu, Y.; Talley, J. J. Synthesis of Deuterated Benzopyran Derivatives as Selective COX-2 Inhibitors with Improved Pharmacokinetic Properties. ACS Med. Chem. Lett. 2014, 5, 1162-1166. (d) Reddy K. R.; Rao P. S.; Dev G. J.; Poornachandra, Y.; Kumar, C. G.; Rao, P. S.; Narsaiah, B. Synthesis of novel 1,2,3-triazole/isoxazole functionalized 2H-Chromene derivatives and their cytotoxic activity. Bioorg. Med. Chem. Lett. 2014, 24, 1661-1663. (e) Zhang, Y.; Wang, Y.; He, C.; Liu, X.; Lu, Y.; Chen, T.; Pan, Q.; Xiong, J.; She, M.; Tu, Z.; Qin, X.; Li, M.; Tortorella, M. D.; Talley, J. J. Pentafluorosulfanyl-Substituted Benzopyran Analogues As New Cyclooxygenase-2 Inhibitors with Excellent Pharmacokinetics and Efficacy in Blocking Inflammation. J. Med. Chem. 2017, 60, 4135-4146.

${ }^{25} \mathrm{Here}$, TfOH was found to be a slightly better catalyst than $\mathrm{FeCl}_{3}$. For more information, see the SI.

${ }^{26}$ Wabnitz, T. C.; Yu, J. Q.; Spencer, J. B. Evidence That Protons Can Be the Active Catalysts in Lewis Acid Mediated Hetero-Michael Addition Reactions. Chem. Eur. J. 2004, 10, 484-493.

${ }^{27}$ Zhang, X.; Teo, W. T.; Hong Chan, P. W. Ytterbium(III) Triflate Catalyzed Tandem Friedel-Crafts Alkylation/Hydroarylation of Propargylic Alcohols with Phenols as an Expedient Route to Indenols. Org. Lett. 2009, 11, 4990-4993.

${ }^{28}$ Cordier, P.; Aubert, C.; Malacria, M.; Lacôte, E.; Gandon, V. Silver and Brønsted Acid Catalyzed Nazarov-Type Cyclizations To Generate Benzofulvenes. Angew. Chem. Int. Ed. 2009, 48, 8757-8760.

${ }^{29}$ Geri, J. B.; Wade Wolfe, M. M.; Szymczak, N. K. Borazine-CF 3 - Adducts for Rapid, Room Temperature, and Broad Scope Trifluoromethylation. Angew. Chem. Int. Ed. 2018, 57, 1381-1385.

${ }^{30}$ Zheng, Y.; Tan, Y.; Harms, K.; Marsch, M, Riedel, R.; Zhang, L.; Meggers, E. Ctahedral Ruthenium Complex with Exclusive Metal-Centered Chirality for Highly Effective Asymmetric Catalysis. J. Am. Chem. Soc. 2017, 139, 43224325.

${ }^{31}$ Irudayanathan, F. M.; Kim, J.; Song, K. H.; Lee, S. Transition-Metal-Free Decarboxylative Coupling Reactions for the Synthesis of Propargyl Alcohols. Asian J. Org. Chem. 2016, 5, 1148-1154 
${ }^{32}$ Motoki R.; Kanai M.; Shibasaki M. Copper(I) Alkoxide-Catalyzed Alkynylation of Trifluoromethyl Ketones. Org. Lett. 2007, 9, 2997-3000

${ }^{33}$ Qiu Y.-F. et al. Convenient and Highly Efficient Routes to 2 H-Chromene and 4-Chromanone Derivatives: IodinePromoted and p-Toluenesulfonic Acid Catalyzed Cascade Cyclizations of Propynols. Chem. Eur. J. 2015, 21, 34803487.

${ }^{34}$ (a) Kitazume, T.; Sato, T. A synthetic approach to seven-membered lactones by the microbial transformation of ynones having a trifluoromethyl group. J. Fluorine Chem. 1985, 30, 189-202. (b) Xiao, L. et al. Optically active propargylic and allylic alcohols with a difluoromethyl group at the terminal carbon. Tetrahedron: Asymmetry 1997, 8, 3597-3601.

${ }^{35}$ Ko, S.-J. Kinetic resolution of fluorinated propargyl alcohols by lipase-catalyzed enantioselective transesterification. Tetrahedron: Asymmetry 2009, 20, 1109-1114. 